\title{
ABSOLUTE CONTINUITY OF PARABOLIC MEASURE AND THE INITIAL-DIRICHLET PROBLEM
}

\author{
A Dissertation \\ presented to \\ the Faculty of the Graduate School \\ University of Missouri
}

In Partial Fulfillment

of the Requirements for the Degree

Doctor of Philosophy

by

ALYSSA GENSCHAW

Dr. Steve Hofmann, Dissertation Supervisor

MAY, 2019 
The undersigned, appointed by the Associate Vice Chancellor of the Office of Research and Graduate Studies, have examined the dissertation entitled

\section{ABSOLUTE CONTINUITY OF PARABOLIC MEASURE AND THE INITIAL-DIRICHLET PROBLEM}

presented by Alyssa Genschaw, a candidate for the degree of Doctor of Philosophy of Mathematics, and hereby certify that, in their opinion, it is worthy of acceptance.

Professor Steve Hofmann

Professor Marius Mitrea

Professor Samuel Walsh

Professor Steven Neal 


\section{ACKNOWLEDGEMENTS}

I would like to start out by thanking my parents, who have been my biggest cheerleaders since day one. This dissertation would not have been possible without their constant reassurance, positivity, and encouragement. A special thank you to my father, who has always been my role model and who has helped me tremendously in this process.

I am thankful for my husband, Chase, who stood by my side as I followed my dreams. He has made each move an adventure and has been my escape throughout this journey.

I am forever grateful to my advisor, Steve Hofmann, for his guidance, knowledge, and support.

Finally, I would like to thank my daughter, Audrey, for being my source of determination in finishing this dissertation. 


\section{Contents}

Acknowledgements

$\begin{array}{ll}\text { Abstract } & \text { v }\end{array}$

1 Introduction 1

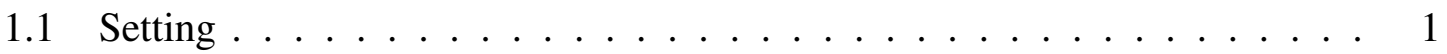

1.2 Notation \& Definitions . . . . . . . . . . . . . . . 2

2 Preliminary Results $\quad 15$

2.0.1 Perron Solution . . . . . . . . . . . . . . . 17

2.0.2 Parabolic Bourgain-type Estimates . . . . . . . . . . . . . . . 19

2.0.3 Hölder Continuity at the Boundary . . . . . . . . . . . . . . 28

2.0.4 Boundary Harnack . . . . . . . . . . . . . . . . . . . . 31

2.0.5 Caccioppoli's Inequality . . . . . . . . . . 36

3 Main Results $\quad 41$

3.1 A Weak Reverse Hölder Inequality for Parabolic Measure . . . . . . . . . . 43

3.1 .1 Introduction . . . . . . . . . . . . . 43

3.1 .2 Main Result . . . . . . . . . . . . . . . . . . . . 44

3.1.3 Lemmas \& Proof . . . . . . . . . . . . . . . . . . 47

3.1 .4 Applications ....................... 64 


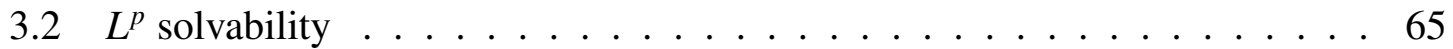

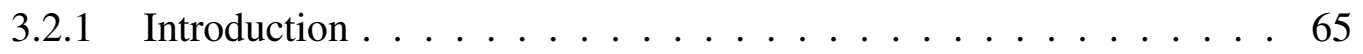

3.2.2 Main Result . . . . . . . . . . . . . . . . . . . . . . . 66

3.3 BMO Solvability $\ldots \ldots \ldots \ldots \ldots \ldots \ldots \ldots$

3.3 .1 Introduction $\ldots \ldots \ldots \ldots \ldots \ldots \ldots$

3.3.2 Main Result . . . . . . . . . . . . . . . . . . . . . . . . 77

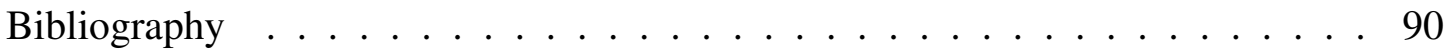

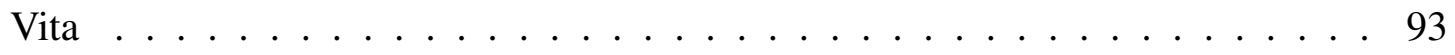




\begin{abstract}
This thesis is devoted to the study of parabolic measure corresponding to a divergence form parabolic operator. We first extend to the parabolic setting a number of basic results that are well known in the elliptic case. Then following a result of Bennewitz-Lewis for non-doubling harmonic measure, we prove a criterion for non-doubling caloric measure to satisfy a weak reverse Hölder inequality on an open set $\Omega \subset \mathbb{R}^{n+1}$, assuming as a background hypothesis only that the essential boundary of $\Omega$ satisfies an appropriate parabolic version of Ahlfors-David regularity (which entails some backwards in time thickness). We then show that the weak reverse Hölder estimate is equivalent to solvability of the initial Dirichlet problem with "lateral" data in $L^{p}$, for some $p<\infty$. Finally, we prove that for the heat equation, BMO-solvability implies scale invariant quantitative absolute continuity of caloric measure with respect to surface measure, in an open set $\Omega$ with time-backwards ADR boundary. Moreover, the same results apply to the parabolic measure associated to a uniformly parabolic divergence form operator $L$, with estimates depending only on dimension, the ADR constants, and parabolicity, provided that the continuous Dirichlet problem is solvable for $L$ in $\Omega$. By a result of Fabes, Garofalo and Lanconelli [FGL], this includes the case of $C^{1}$-Dini coefficients.
\end{abstract}




\section{Chapter 1}

\section{Introduction}

\subsection{Setting}

We assume that $\Omega \subset \mathbb{R}^{n+1}$ is an open set whose boundary satisfies an appropriate version of a parabolic Ahlfors-David regularity condition.

We shall consider the heat operator

$$
L_{0}:=\partial_{t}-\mathcal{L}
$$

where $\mathcal{L}:=\nabla \cdot \nabla$ is the usual Laplacian in $\mathbb{R}^{n}$, acting in the space variables. With a caveat, to be discussed in Chapter 3, our results may apply more generally to divergence form parabolic operators

$$
L:=\partial_{t}-\operatorname{div} A(X, t) \nabla,
$$

defined in an open set $\Omega \subset \mathbb{R}^{n+1}$ as described above, where $A$ is $n \times n$, real, $L^{\infty}$, and satisfies the uniform ellipticity condition

$$
\lambda|\xi|^{2} \leq\langle A(X, t) \xi, \xi\rangle:=\sum_{i, j=1}^{n+1} A_{i j}(X, t) \xi_{j} \xi_{i}, \quad\|A\|_{L^{\infty}\left(\mathbb{R}^{n}\right)} \leq \lambda^{-1},
$$

for some $\lambda>0$, and for all $\xi \in \mathbb{R}^{n}$, and a.e. $(X, t) \in \Omega$. We do not require that the matrix $A(X, t)$ be symmetric. We reference the paper by Moser $[\mathrm{M}]$, where the results are stated under an assumption of symmetry, but in fact symmetry is not needed: see [DK], [SSSZ], $[\mathrm{QX}]$. 


\subsection{Notation \& Definitions}

For a set $A \subset \mathbb{R}^{n+1}$, we define

$$
T_{\text {min }}(A):=\inf \{T: A \cap\{t \equiv T\} \neq \emptyset\}, \quad T_{\max }(A):=\sup \{T: A \cap\{t \equiv T\} \neq \emptyset\}
$$

(note: it may be that $T_{\min }(A)=-\infty$, and/or that $T_{\max }(A)=+\infty$ ). In the special case that $A=\Omega$, an open set that has been fixed, we will simply write $T_{\min }=T_{\min }(\Omega)$ and $T_{\max }=T_{\max }(\Omega)$.

Definition 1.2.2 (Parabolic cubes). An (open) parabolic cube in $\mathbb{R}^{n} \times \mathbb{R}$ with center $(X, t)$ :

$$
\begin{aligned}
& Q_{r}(X, t):=Q((X, t), r) \\
& \quad:=\left\{(Y, s) \in \mathbb{R}^{n} \times \mathbb{R}:\left|X_{i}-Y_{i}\right|<r, 1 \leq i \leq n, t-r^{2}<s<t+r^{2}\right\} .
\end{aligned}
$$

With a mild abuse of terminology, we refer to $r$ as the "parabolic sidelength" (or simply the "length") of $Q_{r}(X, t)$. We shall sometimes simply write $Q_{r}$ to denote a cube of parabolic length $r$, when the center is implicit, and for $Q=Q_{r}$, we shall write $\ell(Q)=r$.

We also consider the time-backward and time-forward versions:

$$
\begin{aligned}
& Q^{-}((X, t), r):=Q_{r}^{-}(X, t) \\
& \quad:=\left\{(Y, s) \in \mathbb{R}^{n} \times \mathbb{R}:\left|X_{i}-Y_{i}\right|<r, 1 \leq i \leq n, t-r^{2}<s<t\right\}, \\
& Q^{+}((X, t), r):=Q_{r}^{+}(X, t) \\
& \quad:=\left\{(Y, s) \in \mathbb{R}^{n} \times \mathbb{R}:\left|X_{i}-Y_{i}\right|<r, 1 \leq i \leq n, t<s<t+r^{2}\right\} .
\end{aligned}
$$

We shall sometimes also use the letter $P$ to denote parabolic cubes in $\mathbb{R}^{n+1}$. 
Definition 1.2.4 (Classification of boundary points). Following [L], given an open set $\Omega \subset \mathbb{R}^{n+1}$, we define its parabolic boundary $\mathcal{P} \Omega$ as

$$
\mathcal{P} \Omega:=\left\{(x, t) \in \partial \Omega: \forall r>0, Q_{r}^{-}(x, t) \text { meets } \mathbb{R}^{n+1} \backslash \Omega\right\}
$$

The bottom boundary, denoted $\mathcal{B} \Omega$, is defined as

$$
\mathcal{B} \Omega:=\left\{(x, t) \in \mathcal{P} \Omega: \exists \varepsilon>0 \text { such that } Q_{\varepsilon}^{+}(x, t) \subset \Omega\right\} .
$$

The lateral boundary, denoted $\mathcal{S} \Omega$, is defined as

$$
\mathcal{S} \Omega:=\mathcal{P} \Omega \backslash \mathcal{B} \Omega
$$

Following [W1, W2], we also define the normal boundary, denoted $\partial_{n} \Omega$, to be equal to the parabolic boundary in a bounded domain, while in an unbounded domain, we append the point at infinity: $\partial_{n} \Omega=\mathcal{P} \Omega \cup\{\infty\}$. The abnormal boundary is defined as $\partial_{a} \Omega:=\partial \Omega \backslash \partial_{n} \Omega$, thus:

$$
\partial_{a} \Omega:=\left\{(x, t) \in \partial \Omega: \exists \varepsilon>0 \text { such that } Q_{\varepsilon}^{-}(x, t) \subset \Omega\right\} \text {. }
$$

The abnormal boundary is further decomposed into $\partial_{a} \Omega=\partial_{s} \Omega \cup \partial_{s s} \Omega$ (the singular boundary and semi-singular boundary, respectively), where

$$
\partial_{s} \Omega:=\left\{(x, t) \in \partial_{a} \Omega: \exists \varepsilon>0 \text { such that } Q_{\varepsilon}^{+}(x, t) \cap \Omega=\emptyset\right\}
$$

and

$$
\partial_{s s} \Omega:=\left\{(x, t) \in \partial_{a} \Omega: \forall r>0 Q_{r}^{+}(x, t) \text { meets } \Omega\right\} .
$$

The essential boundary $\partial_{e} \Omega$, is defined as

$$
\partial_{e} \Omega:=\partial_{n} \Omega \cup \partial_{s s} \Omega=\partial \Omega \backslash \partial_{s} \Omega
$$


(where we replace $\partial \Omega$ by $\partial \Omega \cup\{\infty\}$ if $\Omega$ is unbounded). Finally, we define the quasi-lateral boundary $\Sigma$ to be

$$
\Sigma:=\left\{\begin{array}{l}
\partial \Omega, \quad \text { if } T_{\text {min }}=-\infty \text { and } T_{\max }=\infty \\
\partial \Omega \backslash(\mathcal{B} \Omega)_{T_{\min }}, \quad \text { if } T_{\min }>-\infty \text { and } T_{\max }=\infty \\
\partial \Omega \backslash\left(\partial_{s} \Omega\right)_{T_{\max }}, \quad \text { if } T_{\text {max }}<\infty \text { and } T_{\min }=-\infty \\
\partial \Omega \backslash\left((\mathcal{B} \Omega)_{T_{\min }} \cup\left(\partial_{s} \Omega\right)_{T_{\max }}\right), \quad \text { if }-\infty<T_{\min }<T_{\max }<\infty .
\end{array}\right.
$$

where $(\mathcal{B} \Omega)_{T_{\min }}$ is the time slice of $\mathcal{B} \Omega$ with $t \equiv T_{\min }$, and $\left(\partial_{s} \Omega\right)_{T_{\max }}$ is the time slice of $\partial_{s} \Omega$ with $t \equiv T_{\max }$. Observe that for a cylindrical domain $\Omega=U \times\left(T_{\min }, T_{\max }\right)$, with $U \subset \mathbb{R}^{n}$ a domain in the spatial variables, then $\Sigma$ would simply be the usual lateral boundary.

- We use the letters $c, C$ to denote harmless positive constants, not necessarily the same at each occurrence, which depend only on dimension and the constants appearing in the hypotheses of the theorems (which we refer to as the "allowable parameters"). We shall also sometimes write $a \lesssim b$ and $a \approx b$ to mean, respectively, that $a \leq C b$ and $0<c \leq a / b \leq C$, where the constants $c$ and $C$ are as above, unless explicitly noted to the contrary.

- We shall use lower case letters $x, y, z$, etc., to denote the spatial component of points on the boundary $\partial \Omega$, and capital letters $X, Y, Z$, etc., to denote the spatial component of generic points in $\mathbb{R}^{n+1}$ (in particular those in $\Omega$ ).

- For the sake of notational brevity, we shall sometimes also use boldface capital letters to denote points in space time $\mathbb{R}^{n+1}$, and lower case boldface letters to denote points on $\partial \Omega$; thus,

$$
\mathbf{X}=(X, t), \quad \mathbf{Y}=(Y, s), \quad \text { and } \quad \mathbf{x}=(x, t), \quad \mathbf{y}=(y, s)
$$

- We shall orient our coordinate axes so that time runs from left to right. 
- We let $\mathbb{S}^{n}$ denote the unit sphere in $\mathbb{R}^{n+1}$.

- $\mathcal{H}^{d}$ denotes $d$-dimensional Hausdorff measure.

- For $A \subset \mathbb{R}^{n+1}$, let $A_{s}:=\{(X, t) \in A: t \equiv s\}$ denote the time slice of $A$ with $t \equiv s$.

- We let $d \sigma=d \sigma_{s} d s$ denote the "surface measure" on the quasi-lateral boundary $\Sigma$, where $d \sigma_{s}:=\left.\mathcal{H}^{n-1}\right|_{\Sigma_{s}}$, and $\Sigma_{s}$ is the time slice of $\Sigma$, with $t \equiv s$. See Remark 1.2.22 for some clarifying comments.

- The parabolic norm of $\mathbf{X}=(X, t) \in \mathbb{R}^{n+1}$, denoted $\|\mathbf{X}\|$, is the unique solution $\rho$ of the equation

$$
\frac{|X|^{2}}{\rho^{2}}+\frac{t^{2}}{\rho^{4}}=1 .
$$

We observe that the parabolic norm satisfies

$$
\|\mathbf{X}\|=\|(X, t)\| \approx|X|+|t|^{1 / 2}
$$

The parabolic $\ell^{\infty}$ norm is defined by

$$
\|\mathbf{X}\|_{\ell^{\infty}}=\|(X, t)\|_{\ell^{\infty}}:=\max \left\{\left|X_{1}\right|, \ldots,\left|X_{n}\right|,|t|^{1 / 2}\right\}
$$

Of course, the parabolic norm and parabolic $\ell^{\infty}$ norm induce corresponding distances on $\mathbb{R}^{n+1}$, which are comparable to each other.

- If $\mathbf{X} \in \Omega$, we let $\delta(\mathbf{X}):=\operatorname{dist}\left(\mathbf{X}, \partial_{e} \Omega\right)$, and $\delta_{\infty}(\mathbf{X}):=\operatorname{dist}_{\infty}\left(\mathbf{X}, \partial_{e} \Omega\right)$, denote the parabolic distance, respectively, the parabolic $\ell^{\infty}$ distance, to the essential boundary. We note that $\delta_{\infty}(X, t) \approx \delta(X, t)$, with uniform implicit constants depending only on dimension. 
Definition 1.2.10. Given $\mathbf{X} \in \Omega$, a touching point for $\mathbf{X}$ is any point $\hat{\mathbf{x}} \in \partial_{e} \Omega$ such that $\|\mathbf{X}-\hat{\mathbf{x}}\|=\delta(\mathbf{X})$. The "touching ball" for $\mathbf{X}$ is the parabolic ball

$$
B_{r}(\mathbf{X}):=\left\{\mathbf{Y} \in \mathbb{R}^{n+1}:\|\mathbf{X}-\mathbf{Y}\|<r\right\}
$$

with $r=\delta(\mathbf{X})$.

At times, we shall also find it convenient to work with "touching cubes" and "touching points" with respect to the parabolic $\ell^{\infty}$ distance to the essential boundary:

Definition 1.2.11. Given a point $(X, t) \in \Omega$, let $Q_{\star}(X, t)$ denote the "touching cube" for the point $(X, t)$, i.e., set $Q_{\star}(X, t):=Q_{r}(X, t)$, where

$$
r=r_{\star}(X, t):=\sup \left\{\rho>0: Q_{\rho}(X, t) \cap \partial_{e} \Omega=\emptyset\right\}
$$

so that (since our cubes are open) $Q_{\star}(X, t) \cap \partial_{e} \Omega=\emptyset$, and $\partial Q_{\star}(X, t)$ meets $\partial_{e} \Omega$. We shall say that $(\hat{x}, \hat{t}) \in \partial_{e} \Omega$ is a "touching point in the $\ell^{\infty}$ sense" (or just a "touching point", if it is implicit that we are working with the $\ell^{\infty}$ metric) for $(X, t)$, if $(\hat{x}, \hat{t}) \in$ $\partial Q_{\star}(x, t) \cap \partial_{e} \Omega$. Note that $\delta_{\infty}(X, t):=r_{\star}(X, t)$.

We further note that if $\sqrt{t-T_{\min }}>\delta_{\infty}(X, t)$, then $(\hat{x}, \hat{t}) \in \Sigma$, for any touching point $(\hat{x}, \hat{t})$ of $(X, t)$, i.e., in this case $\delta_{\infty}(X, t)=\operatorname{dist}_{\infty}((X, t), \Sigma)$.

- For a set $A \subset \mathbb{R}^{n+1}$, we shall write $\operatorname{diam}(A)$ to denote the diameter of $A$ with respect to the parabolic distance, i.e.,

$$
\operatorname{diam}(A):=\sup _{((X, t),(Y, s)) \in A \times A}\|(X, t)-(Y, s)\| .
$$

- Given a Borel measure $\mu$, and a Borel set $A \subset \mathbb{R}^{n}$, with positive and finite $\mu$ measure, we set $f_{A} f d \mu:=\mu(A)^{-1} \int_{A} f d \mu$; if $A$ is a subset of space-time $\mathbb{R}^{n+1}$, we then write $f_{A} f d \mu:=\mu(A)^{-1} \iint_{A} f(X, t) d \mu(X, t)$. 
- A "surface cube" on $\Sigma$ is defined by

$$
\Delta=Q \cap \Sigma,
$$

where $Q$ is a parabolic cube centered on $\Sigma$, or more precisely,

$$
\Delta=\Delta_{r}(x, t):=Q_{r}(x, t) \cap \Sigma,
$$

with $(x, t) \in \Sigma$. We note that the "surface cubes" are not the same as the dyadic cubes of M. Christ [Ch] on $\Sigma$; we apologize to the reader for the possibly confusing terminology.

Definition 1.2.13. We define the following boundary value problems. The second is relevant only in the case that $T_{\min }=-\infty$.

I. Continuous Dirichlet Problem:

$$
\text { (D) }\left\{\begin{aligned}
L u & =0 \text { in } \Omega \\
\left.u\right|_{\partial_{e} \Omega} & =f \in C_{c}\left(\partial_{e} \Omega\right) \\
u & \in C\left(\Omega \cup \partial_{n} \Omega\right) .
\end{aligned}\right.
$$

If $\Omega$ is unbounded, we further specify that $\lim _{\|\mathbf{X}\| \rightarrow \infty} u(\mathbf{X})=0$. Here, we interpret the statement $\left.u\right|_{\partial_{e} \Omega}=f \in C_{c}\left(\partial_{e} \Omega\right)$ to mean that

$$
\lim _{(X, t) \rightarrow(y, s)} u(X, t)=f(y, s), \quad(y, s) \in \partial_{n} \Omega,
$$

and

$$
\lim _{(X, t) \rightarrow\left(y, s^{+}\right)} u(X, t)=f(y, s), \quad(y, s) \in \partial_{s s} \Omega .
$$

If the preceeding problem is solvable for all $f \in C_{c}\left(\partial_{e} \Omega\right)$, then we say that the "continuous Dirichlet problem is solvable for $L . "$

II. $L^{p}$ Dirichlet Problem: 


$$
(D)_{p} \begin{cases}L u & =0 \text { in } \Omega \\ \left.u\right|_{\Sigma} & =f \in L^{p}(\Sigma) \\ & N_{*} u \in L^{p}(\Sigma) .\end{cases}
$$

III. Continuous Initial-Dirichlet Problem:

$$
(I-D)\left\{\begin{aligned}
L u & =0 \text { in } \Omega^{T}:=\Omega \cap\{t>T\} \\
u(X, T) & =0 \text { in } \Omega_{T}=\Omega \cap\{t \equiv T\} \\
\left.u\right|_{\Sigma^{T}} & =f \in C_{c}\left(\Sigma^{T}\right) \\
& u \in C\left(\Omega^{T} \cup \partial_{n} \Omega^{T}\right) .
\end{aligned}\right.
$$

Here, $\Sigma^{T}$ denotes the quasi-lateral boundary of the domain $\Omega^{T}$. The statement $\left.u\right|_{\Sigma^{T}}=$ $f \in C_{c}\left(\Sigma^{T}\right)$ is intepreted as in problem $\mathrm{I}$, and if $\Omega^{T}$ is unbounded, we further specify that $\lim _{\|\mathbf{X}\| \rightarrow \infty} u(\mathbf{X})=0$.

IV. $L^{p}$ Initial-Dirichlet Problem:

$$
(I-D)_{p}\left\{\begin{aligned}
L u & =0 \text { in } \Omega^{T}:=\Omega \cap\{t>T\} \\
u(X, T) & =0 \text { in } \Omega_{T}=\Omega \cap\{t \equiv T\} \\
\left.u\right|_{\Sigma^{T}} & =f \in L^{p}\left(\Sigma^{T}\right) \\
& N_{*} u \in L^{p}\left(\Sigma^{T}\right) .
\end{aligned}\right.
$$

In problems II and IV, the statement $\left.u\right|_{\Sigma}=f \in L^{p}(\Sigma)$ (resp., $\left.u\right|_{\Sigma^{T}}=f \in L^{p}\left(\Sigma^{T}\right)$ ) is understood in the sense of parabolic non-tangential convergence. We shall discuss this issue, as well as the precise definition of the non-tangential maximal function $N_{*} u$, in Chapter 3 . In problems III and IV, the statement $u(X, T)=0$ in $\Omega_{T}$ means that $u$ vanishes continuously on $\Omega_{T}$.

Definition 1.2.14. (Caloric and Parabolic Measure) Assume $\Omega \subset \mathbb{R}^{n+1}$ is an open set. Let $u$ be the PWB solution (see [W1], [W2, Chapter 8]) of the Dirichlet problem for the heat equation, with boundary data $f \in C_{c}\left(\partial_{e} \Omega\right)$. By the Perron construction, for each point $(X, t) \in \Omega$, the mapping $f \mapsto u(X, t)$ is bounded, and by the resolutivity of functions $f \in C\left(\partial_{e} \Omega\right)$ (see [W2, Theorem 8.26]), it is also linear. The caloric measure with pole $(X, t)$ 
is the probability measure $\omega^{X, t}$ given by the Riesz representation theorem, such that

$$
u(X, t)=\iint_{\partial_{e} \Omega} f(y, s) d \omega^{X, t}(y, s)
$$

For a general divergence form parabolic operator $L$ as in (1.1.2)-(1.1.3), parabolic measure $\omega^{X, t}=\omega_{L}^{X, t}$ may be defined similarly, provided that the continuous Dirichlet problem is solvable for $L$.

Remark 1.2.16. Caloric measure is supported on the essential boundary; see [Su], or [W1, W2].

Definition 1.2.17. (ADR) (aka Ahlfors-David regular [in the parabolic sense]). Let $\Omega \subset$ $\mathbb{R}^{n+1}$. We say that the quasi-lateral boundary $\Sigma$ is globally $A D R$ (or just ADR) if there is a constant $M_{0}$ such that for every parabolic cube $Q_{r}=Q_{r}(x, t)$, centered on $\Sigma$, and corresponding surface cube $\Delta_{r}=Q_{r} \cap \Sigma$, with $r<\operatorname{diam}(\Omega)$,

$$
\frac{1}{M_{0}} r^{n+1} \leq \sigma\left(\Delta_{r}\right) \leq M_{0} r^{n+1}
$$

We also say that $\Sigma$ is ADR on a surface cube $\Delta=Q \cap \Sigma$, if there is a constant $M_{0}$ such that (1.2.18) holds for every surface cube $\Delta_{r}=Q_{r} \cap \Sigma$, with $Q_{r} \subset Q$ and centered on $\Sigma$.

Definition 1.2.19. (Time-Backwards ADR, aka TBADR) Given a parabolic cube $Q$ centered on $\Sigma$, and corresponding surface cube $\Delta=Q \cap \Sigma$, we say that $\Sigma$ is time-backwards ADR on $\Delta$ if it is ADR on $\Delta$, and if, in addition there exists a constant $b_{0}>0$ such that

$$
b_{0} r^{n+1} \leq \sigma\left(\Delta_{r}^{-}\right)
$$

for every $\Delta_{r}^{-}=Q_{r}^{-} \cap \Sigma$, where $Q_{r} \subset Q$ is centered at some point $(x, t) \in \Sigma$.

If $\Sigma$ is time-backwards ADR on every $\Delta=\Sigma \cap Q_{r}\left(x_{0}, t_{0}\right)$, for all $\left(x_{0}, t_{0}\right) \in \Sigma$, and for all $r$ with $0<r<\min \left(\operatorname{diam}(\Sigma), \sqrt{t_{0}-T_{\min }} /(4 \sqrt{n})\right)$, then we shall simply say that $\Sigma$ is (globally) 
time-backwards ADR (and we shall refer to such $\Delta$ as "admissible"; note that if $T_{\min }=-\infty$, then there is no restriction on $r$, and in that case every surface cube is admissible).

Remark 1.2.21. The assumption of some backwards in time thickness, as in Definition 1.2.19, is rather typical in the parabolic setting. See, e.g., the backwards in time capacitary conditions in [La], [EG], [GL], [FGL], [GZ], [BiM]. Moreover, it is not hard to verify that by the result of $[\mathrm{EG}]$ (or of [GL], [FGL]), time-backwards ADR on some surface cube $\Delta$ implies parabolic Wiener-type regularity of each point in $\Delta$ (and thus global timebackwards ADR implies regularity of the parabolic boundary $\mathcal{P} \Omega$ ). We refer to Lemma 2.0.2 below for the details.

Remark 1.2.22. By [W2, Theorem 8.40], the abnormal boundary $\partial_{a} \Omega$ is contained in a countable union of hyperplanes orthogonal to the $t$-axis. Moreover, the same is true for the bottom boundary $\mathcal{B} \Omega$, since its image under the change of variable $t \rightarrow-t$ is contained in $\partial_{a} \Omega^{*}$, for the domain $\Omega^{*}$ obtained from $\Omega$ by the same change of variable. Thus, $\sigma(\mathcal{B} \Omega)=$ 0 .

Remark 1.2.23. The time-backwards ADR condition rules out pathologies like a vertical face (with time running from left to right horizontally) on $\Sigma$. In particular, $\partial_{s s} \Omega=\emptyset=$ $\partial_{s} \Omega \backslash\left\{t \equiv T_{\max }\right\}$, at least locally on any surface cube $\Delta$ on which TBADR holds, and thus $\partial_{e} \Omega=\partial_{n} \Omega=\mathcal{P} \Omega=\Sigma$ on such $\Delta$. Moreover, under the hypotheses of Theorem 3.1.3 and Theorem 3.3.2, $\mathcal{B} \Omega \cap \Delta_{r}\left(x_{0}, t_{0}\right)$ is fairly negligible: by Remark 1.2.22, this set has $\sigma$ measure zero, and thus in the setting of Theorem 3.1.3 and Theorem 3.3.2, in which in particular we deduce that $\omega \ll \sigma$, it also has caloric measure zero.

Remark 1.2.24. The significance of the admissibility constraint is as follows. Recall that $\delta(\mathbf{Y}):=\operatorname{dist}\left(\mathbf{Y}, \partial_{e} \Omega\right)$, where the distance is of course the parabolic distance. We note that, 
by elementary geometry and (1.2.6) (i.e., the definition of $\Sigma$ ), for $\left(x_{0}, t_{0}\right) \in \Sigma$, we have that

$$
r<\sqrt{t_{0}-T_{\min }} /(4 \sqrt{n}) \Longrightarrow \delta(\mathbf{Y})=\operatorname{dist}(\mathbf{Y}, \Sigma), \quad \forall \mathbf{Y} \in \Omega \cap Q_{r}\left(x_{0}, t_{0}\right)
$$

Remark 1.2.25. We will show, in Claim 1 of Lemma 2.0.5, that time-backwards ADR yields an apparently stronger property: specifically, we show that if $\Sigma$ is time-backwards ADR on $\Delta=\Delta_{r}=\Sigma \cap Q_{r}\left(x_{0}, t_{0}\right)$, then (1.2.20) continues to hold (with a slightly different constant) with $\Delta_{r}^{-}$replaced by $\Delta_{r}^{-} \cap\left\{t<t_{0}-(a r)^{2}\right\}$, and hence also by $\Sigma \cap Q_{r}^{-}\left(x_{0}, t_{0}-(a r)^{2}\right)$, for some uniform $a \in(0,1 / 2)$.

Definition 1.2.26. (Time-Forward ADR, aka TFADR) Given a parabolic cube $Q$ centered on $\Sigma$, and corresponding surface cube $\Delta=Q \cap \Sigma$, we say that $\Sigma$ is time-forward ADR on $\Delta$ if it is $\mathrm{ADR}$ on $\Delta$, and if, in addition there exists $b_{0}>0$ such that

$$
b_{0} r^{n+1} \leq \sigma\left(\Delta_{r}^{+}\right)
$$

for every $\Delta_{r}^{+}=Q_{r}^{+} \cap \Sigma$, where $Q_{r} \subset Q$ is centered at some point $(x, t) \in \Sigma$.

If $\Sigma$ is time-forward ADR on every $\Delta=\Sigma \cap Q_{r}\left(x_{0}, t_{0}\right)$, for all $\left(x_{0}, t_{0}\right) \in \Sigma$, and for all $r$ with $0<r<\min \left(\operatorname{diam}(\Sigma), \sqrt{T_{\max }-t_{0}} /(4 \sqrt{n})\right)$, then we shall simply say that $\Sigma$ is time-forward ADR.

Definition 1.2.28. (Time-Symmetric ADR) Given a parabolic cube $Q$ centered on $\Sigma$, and corresponding surface cube $\Delta=Q \cap \Sigma$, we say that $\Sigma$ is time-symmetric ADR on $\Delta$ if it is ADR on $\Delta$, as well as time-backwards ADR on $\Delta$ and time-forward ADR on $\Delta$.

If $\Sigma$ is time-symmetric ADR on every $\Delta=\Sigma \cap Q_{r}\left(x_{0}, t_{0}\right)$, for all $\left(x_{0}, t_{0}\right) \in \Sigma$, and for all $r$ with $0<r<\min \left(\operatorname{diam}(\Sigma), \sqrt{T_{\max }-t_{0}} /(4 \sqrt{n}), \sqrt{t_{0}-T_{\min }} /(4 \sqrt{n})\right)$, then we shall simply say that $\Sigma$ is time-symmetric ADR. 
Definition 1.2.29. (Parabolic BMO). $B M O(\Sigma)$ is the parabolic version of the usual BMO space with norm $\|f\|_{B M O(\Sigma)}$, defined for any locally integrable function $f$ on $\Sigma$ by

$$
\|f\|_{B M O(\Sigma)}:=\sup _{\Delta}\left\{f f_{\Delta}\left|f-f_{\Delta}\right| d \sigma\right\}<\infty
$$

where $\Delta=\Delta_{r}(x, t):=Q_{r}(x, t) \cap \Sigma, f_{\Delta}:=f f_{\Delta} f,(x, t) \in \Sigma$, and $0<r<\operatorname{diam}(\Sigma)$.

Definition 1.2.31. (Parabolic Polar Coordinates). Let $d \sigma_{\mathbb{S}^{n}}$ denote the usual surface measure on the unit sphere $\mathbb{S}^{n}$ in $\mathbb{R}^{n+1}$. We have the parabolic polar coordinate decomposition

$$
(X, t)=\left(\rho \zeta, \rho^{2} \tau\right), \quad d X d t=\rho^{n+1} d \rho d \mu(\zeta, \tau)
$$

where $(\zeta, \tau) \in \mathbb{S}^{n}, \rho=\|(X, t)\|$, and $\mu$ is an appropriately weighted version of surface measure on the sphere; to be precise, $d \mu(\zeta, \tau):=\left(1+\tau^{2}\right) d \sigma_{\mathbb{S}^{n}}(\zeta, \tau)$; see, e.g. [FR1, FR2] or $[\mathrm{R}]$.

Definition 1.2.32. (Parabolic Projection). We denote by $\pi_{\mathrm{par}}(X, t)$ the parabolic projection of $(X, t)$ onto $\mathbb{S}^{n}$, which we define by setting $\pi_{\mathrm{par}}(X, t)=(\zeta, \tau)$, where $(X, t)$ has the parabolic polar coordinate representation

$$
(X, t)=\left(\rho \zeta, \rho^{2} \tau\right)
$$

with $\rho=\|(X, t)\|$, and $(\zeta, \tau) \in \mathbb{S}^{n}$.

Definition 1.2.33. (Parabolic Cone) Let $(\zeta, \tau) \in \mathbb{S}^{n}$, and let $\vartheta>0$. We define the parabolic cone $\Gamma_{\vartheta}(\zeta, \tau)$, "in the direction $(\zeta, \tau)$ ", with vertex at the origin and aperture $\vartheta>0$, as follows:

$$
\Gamma_{\vartheta}(\zeta, \tau):=\left\{(Y, s):\left\|\pi_{\mathrm{par}}(Y, s)-(\zeta, \tau)\right\|<\vartheta\right\}
$$

For any $(X, t) \in \mathbb{R}^{n+1}$ with $\pi_{\mathrm{p} a r}(X, t)=(\zeta, \tau)$, we shall also write

$$
\Gamma_{\vartheta}(X, t):=\Gamma_{\vartheta}(\zeta, \tau)
$$


Definition 1.2.34. (Harnack Chain condition) Let $\gamma>0$ and $\left(X_{1}, t_{1}\right),\left(X_{2}, t_{2}\right) \in \Omega$ with $\left(t_{2}-t_{1}\right)^{1 / 2}>\gamma^{-1}\left\|\left(X_{1}, t_{1}\right)-\left(X_{2}, t_{2}\right)\right\|$. Then we say that there exists a Harnack Chain $\left\{Q_{r_{i}}\left(Y_{i}, s_{i}\right)\right\}_{i=1}^{k}$ from $\left(X_{1}, t_{1}\right)$ to $\left(X_{2}, t_{2}\right)$ with constant $\gamma$, provided there exists $C(\gamma) \geq 1$ such that

1. $\left(X_{1}, t_{1}\right) \in Q_{r_{1}}\left(Y_{1}, s_{1}\right),\left(X_{2}, t_{2}\right) \in Q_{r_{k}}\left(Y_{k}, s_{k}\right)$,

2. $Q_{r_{i}+1}\left(Y_{i+1}, s_{i+1}\right) \cap Q_{r_{i}}\left(Y_{i}, s_{i}\right) \neq \emptyset$ for $i=1,2, \ldots, k-1$,

3. $\delta\left(Y_{i}, s_{i}\right) \approx r_{i}$, with constants depending on $C(\gamma)$ for $i=1,2, \ldots, k$,

4. $s_{i+1}-s_{i} \geq \frac{r_{i}^{2}}{C(\gamma)}$ for $i=1,2, \ldots, k$

5. $k \leq C(\gamma) \log \left(2+\frac{\left\|\left(X_{1}, t_{1}\right)-\left(X_{2}, t_{2}\right)\right\|}{\min \left\{\delta\left(X_{1}, t_{1}\right), \delta\left(X_{2}, t_{2}\right)\right\}}\right)$.

$k$ is referred to ask the length of the Harnack Chain. If we can do this for every pair of points $\left(X_{1}, t_{1}\right),\left(X_{2}, t_{2}\right) \in \Omega$ with $\left(t_{2}-t_{1}\right)^{1 / 2}>\gamma^{-1}\left\|\left(X_{1}, t_{1}\right)-\left(X_{2}, t_{2}\right)\right\|$ then we say that $\Omega$ satisfies the Harnack Chain condition.

Definition 1.2.35. (Interior Corkscrews) We say that $\Omega$ has interior corkscrews if for some uniform constant $\zeta>0$ and for every surface cube $\Delta_{r}\left(x_{0}, t_{0}\right):=Q_{r}\left(x_{0}, t_{0}\right) \cap \Sigma$ with $\left(x_{0}, t_{0}\right) \in \Sigma$, there exists $(Y, s) \in \Omega \cap Q_{r}\left(x_{0}, t_{0}\right)$ such that $\delta(Y, s) \geq \zeta^{-1} r$.

Definition 1.2.36. (Time-Forward Interior Corkscrews) We say that $\Omega$ has time-forward interior corkscrews if for some uniform constant $\zeta>0$ and for every surface cube $\Delta_{r}\left(x_{0}, t_{0}\right):=$ $Q_{r}\left(x_{0}, t_{0}\right) \cap \Sigma$ with $\left(x_{0}, t_{0}\right) \in \Sigma$, and $0<r<\sqrt{T_{\max }-t_{0}} /(4 \sqrt{n})$, there exists $(Y, s) \in$ $\Omega \cap Q_{r}\left(x_{0}, t_{0}\right)$ such that $\delta(Y, s) \geq \zeta^{-1} r$ and $s=t_{0}+\zeta r^{2}$.

Definition 1.2.37. $\left(A_{\infty}\right.$, weak- $A_{\infty}$, and weak- $\left.R H_{q}\right)$. Given a closed parabolic ADR set $E \subset \mathbb{R}^{n+1}$, and a surface cube $\Delta_{0}:=Q_{0} \cap E$, we say that a Borel measure $\mu$ defined on $E$ 
belongs to weak- $A_{\infty}\left(\Delta_{0}\right)$ if for each surface cube $\Delta=Q \cap E$, with $2 Q \subseteq Q_{0}$,

$$
\mu(F) \leq C\left(\frac{\sigma(F)}{\sigma(\Delta)}\right)^{\theta} \mu(2 \Delta), \quad \text { for every Borel set } F \subset \Delta .
$$

We recall that, as is well known, the condition $\mu \in$ weak- $A_{\infty}\left(\Delta_{0}\right)$ is equivalent to the property that $\mu \ll \sigma$ in $\Delta_{0}$, and that for some $q>1$, the Radon-Nikodym derivative $k:=d \mu / d \sigma$ satisfies the weak reverse Hölder estimate

$$
\left(f_{\Delta} k^{q} d \sigma\right)^{1 / q} \leq C \oiiint_{2 \Delta} k d \sigma \approx \frac{\mu(2 \Delta)}{\sigma(\Delta)}, \quad \forall \Delta=Q \cap E \text {, with } 2 Q \subseteq Q_{0} .
$$

We shall refer to the inequality in (1.2.39) as an " $R H_{q}$ " estimate, and we shall say that $k \in R H_{q}\left(\Delta_{0}\right)$ if $k$ satisfies (1.2.39).

If (1.2.38) holds with $\mu(\Delta)$ in place of $\mu(2 \Delta)$, for all $Q \subset Q_{0}$, then we say that $\mu \in$ $A_{\infty}\left(\Delta_{0}\right)$

Definition 1.2.40. (Subsolution/Supersolution) Following [L], a $C(\bar{\Omega})$ function $v$ will be called a subsolution (supersolution) of $(D)$ if $v \leq f(v \geq f)$ on $\mathcal{P} \Omega$ (we recall that $f \in$ $C\left(\partial_{e} \Omega\right)$ is the prescribed data for the problem $(D)$ in Definition 1.2.13), and if for any ball $B \subset \Omega$ and every solution $u$ in $B, v \leq u(v \geq u)$ in $B$, provided that $v \leq u(v \geq u)$ on $\mathcal{P} B$.

Definition 1.2.41. (Sobolev spaces). Given an open set $U \subset \mathbb{R}^{n+1}$, we define certain Sobolev spaces in $U$ as follows.

$$
W_{2}^{1,1}(U):=\left\{f \in L^{2}(U): \nabla_{x, t} f \in L^{2}(U)\right\}, \quad W_{2}^{1,0}(U):=\left\{f \in L^{2}(U): \nabla_{x} f \in L^{2}(U)\right\}
$$

where the derivatives are understood in the sense of distributions. These spaces are endowed with the respective norms

$$
\|f\|_{W_{2}^{1,1}(U)}:=\|f\|_{L^{2}(U)}+\left\|\nabla_{x, t} f\right\|_{L^{2}(U)}, \quad\|f\|_{W_{2}^{1,0}(U)}:=\|f\|_{L^{2}(U)}+\left\|\nabla_{x} f\right\|_{L^{2}(U)}
$$

We also define $\stackrel{\circ}{W}_{2}^{1,1}(U)$ and $\stackrel{\circ}{W}_{2}^{1,0}(U)$ to be the completions of $C_{0}^{\infty}(U)$ in the $\stackrel{\circ}{W}_{2}^{1,1}$ and $\stackrel{\circ}{W}_{2}^{1,0}(U)$ norms, respectively. 


\section{Chapter 2}

\section{Preliminary Results}

Lemma 2.0.1. The essential boundary $\partial_{e} \Omega$, and the quasi-lateral boundary $\Sigma$, are closed sets.

Proof of Lemma 2.0.1. In the case of $\partial_{e} \Omega$, by definition it is equivalent to show that the singular boundary $\partial_{s} \Omega$ is relatively open in $\partial \Omega$. To this end, fix $\mathbf{x}_{\mathbf{0}}=\left(x_{0}, t_{0}\right) \in \partial_{s} \Omega$, and note that by definition, there is an $\varepsilon>0$ such that

$$
Q_{\varepsilon}^{-}:=Q_{\varepsilon}^{-}\left(\mathbf{x}_{\mathbf{0}}\right) \subset \Omega, \quad \text { and } \quad Q_{\varepsilon}^{+}:=Q_{\varepsilon}^{+}\left(\mathbf{x}_{\mathbf{0}}\right) \subset \mathbb{R}^{n+1} \backslash \Omega \text {. }
$$

Since $Q_{\varepsilon}^{+}$is open, we therefore have that

$$
Q_{\varepsilon}^{+} \subset \operatorname{int}\left(\mathbb{R}^{n+1} \backslash \Omega\right)=: \Omega_{e x t},
$$

where $\operatorname{int}(A)$ denotes the interior of $A$. Consequently, if $\mathbf{x}=(x, t) \in \partial \Omega \cap Q_{\varepsilon}$, where $Q_{\varepsilon}:=Q_{\varepsilon}\left(\mathbf{x}_{\mathbf{0}}\right)$, then $\mathbf{x}$ lies on the interface between $Q_{\varepsilon}^{-}$and $Q_{\varepsilon}^{+}$, i.e., on the time-slice $\left(Q_{\varepsilon}\right)_{t_{0}}$. It follows that for any such $\mathbf{x}$, there is an $\varepsilon^{\prime}=\varepsilon^{\prime}(\mathbf{x})>0$ such that

$$
Q_{\varepsilon^{\prime}}^{-}(\mathbf{x}) \subset \Omega, \quad \text { and } \quad Q_{\varepsilon^{\prime}}^{+}(\mathbf{x}) \subset \mathbb{R}^{n+1} \backslash \Omega,
$$

i.e., $\mathbf{x} \in \partial_{s} \Omega$, by definition, and thus $\partial_{s} \Omega$ is relatively open, as desired.

To see that $\Sigma$ is closed, we first note that $\left(\partial_{s} \Omega\right)_{T_{\max }}$ is relatively open in $\partial \Omega$, by the preceeding argument. Thus, we need only observe in addition that the time slice $(\mathcal{B} \Omega)_{T_{\min }}$ 
is also relatively open in $\partial \Omega$ (assuming that $T_{\min }>-\infty$; otherwise there is nothing to prove). But this follows directly from the fact that under the change of variable $t \rightarrow-t$, which maps $\Omega$ into an open set that we denote $\Omega^{*}$, the time-slice $(\mathcal{B} \Omega)_{T_{\min }}$ is mapped onto $\left(\partial_{s} \Omega^{*}\right)_{T_{\max }\left(\Omega^{*}\right)}$.

Lemma 2.0.2. Assume that $\Sigma$ is time-backwards ADR on $\Delta=\Delta_{r}:=Q_{r} \cap \Sigma$. Then each point $\left(x_{0}, t_{0}\right) \in \Delta$ is regular in the sense of the parabolic Wiener criterion of [EG].

Before beginning the proof of Lemma 2.0.2 we have to introduce some terminology and notation.

Let $K \subset \mathbb{R}^{n+1}$ be a closed set and $\mathcal{M}^{+}(K)$ be the collection of all non-negative Radon measures on $\mathbb{R}^{n+1}$ with support contained in $K$.

Let $W$ denote the standard heat kernel (i.e., Gauss-Weierstrass kernel) on $\mathbb{R}^{n+1}$, and define the thermal capacity of $K$ as

$$
\operatorname{cap}(K):=\sup \left\{\mu\left(\mathbb{R}^{n+1}\right): \mu \in \mathcal{M}^{+}(K), P_{\mu} \leq 1 \text { on } \mathbb{R}^{n+1}\right\},
$$

where

$$
P_{\mu}(X, t):=\iint_{\mathbb{R}^{n+1}} W(X-Y, t-s) d \mu(Y, s) .
$$

Proof of Lemma 2.0.2. Let $\left(x_{0}, t_{0}\right) \in \Delta$, and consider the sets

$$
A_{k}:=A\left(x_{0}, t_{0}, 2^{-k}\right)=\left\{(Y, s) \in \mathbb{R}^{n+1}:\left(4 \pi 2^{-k}\right)^{-n / 2} \leq W\left(x_{0}-Y, t_{0}-s\right) \leq\left(2 \pi 2^{-k}\right)^{-n / 2}\right\}
$$

for $k \in \mathbb{N}$. Using that $Q_{r}$ is an open cube, we restrict our attention to sufficiently large $k$, say $k \geq k_{0}$, with $2^{-k_{0}} \ll \operatorname{dist}\left(\left(x_{0}, t_{0}\right), \partial Q_{r}\right)$, so that $A_{k} \cap \Sigma \subset \Delta$. For any Borel set $E \subset \mathbb{R}^{n+1}$, and for $k \geq k_{0}$ as above, define

$$
\mu_{k}(E):=\frac{\sigma\left(E \cap \Delta \cap A_{k}\right)}{2^{k n / 2} \sigma\left(\Delta \cap A_{k}\right)}=\frac{\sigma\left(E \cap \Sigma \cap A_{k}\right)}{2^{k n / 2} \sigma\left(\Sigma \cap A_{k}\right)} .
$$


By time-backwards ADR, it is a routine matter to check that $\sigma\left(\Sigma \cap A_{k}\right) \approx 2^{-k(n+1) / 2}$. Consequently, for $(X, t) \in \mathbb{R}^{n+1}$,

$$
\begin{aligned}
P_{\mu_{k}}(X, t) & =\iint_{\mathbb{R}^{n+1}} W(X-Y, t-s) d \mu_{k}(Y, s) \\
& =\iint_{\Sigma \cap A_{k}} \frac{W(X-Y, t-s)}{2^{k n / 2} \sigma\left(\Sigma \cap A_{k}\right)} d \sigma(Y, s) \lesssim 2^{k / 2} \iint_{\Sigma \cap A_{k}} W(X-Y, t-s) d \sigma(Y, s) \lesssim 1,
\end{aligned}
$$

where in the last step we have used ADR and the fact that $A_{k} \subset Q_{r_{k}}\left(x_{0}, t_{0}\right)$, with $r_{k} \approx 2^{-k}$. Therefore, for all $k \geq k_{0}$, we have the uniform estimate

$$
\operatorname{cap}\left(\Sigma \cap A_{k}\right) \gtrsim \mu_{k}\left(\mathbb{R}^{n+1}\right) \approx 2^{-k n / 2}
$$

so that, in particular,

$$
\sum_{k=1}^{\infty} 2^{k n / 2} \operatorname{cap}\left(\Omega^{c} \cap A_{k}\right) \geq \sum_{k=k_{0}}^{\infty} 2^{k n / 2} \operatorname{cap}\left(\Sigma \cap A_{k}\right)=+\infty,
$$

verifying the Wiener criterion in [EG].

\subsubsection{Perron Solution}

When studying harmonic functions Oskar Perron introduced a technique for finding a solution of the Dirichlet problem, if the problem is soluble. The technique is referred to as the Perron method (more precisely, the Perron-Wiener-Brelot or "PWB" method) and the solution found is called the Perron (or PWB) solution. The Perron solution is defined by taking the pointwise supremum over the set of all subharmonic functions with boundary values below the desired value. We sketch the argument in [L] to show that the Perron method applies in the parabolic setting.

Theorem 2.0.3 (Perron Solution). Given $f \in C\left(\partial_{e} \Omega\right)$, let $S$ denote the class of all subsolutions $v$, with $v \in C(\bar{\Omega})$, such that $v \leq f$ on $\partial_{e} \Omega$. For $(X, t) \in \Omega$, define

$$
u(X, t)=\sup _{v \in S} v(X, t)
$$


Then $L u=0$ in $\Omega$.

Proof. Note that $v_{0}=-\sup |f|$ is a subsolution of $(D)$, with data $f$, so $u$ is defined for all $(X, t) \in \Omega$ and is bounded from below. Similarly, $-v_{0}$ is a supersolution, so $u$ is bounded from above.

Fix $\left(X_{0}, t_{0}\right) \in \Omega$ and $r$ so that $Q_{r}\left(X_{0}, t_{0}\right) \subset \Omega$, and choose a sequence $\left\{v_{m}\right\}$ of subsolutions such that $v_{m}\left(X_{0}, t_{0}+\frac{r}{8}\right) \rightarrow u\left(X_{0}, t_{0}+\frac{r}{8}\right)$ as $m \rightarrow \infty$.

Define $w_{m}=\max \left\{v_{m}, v_{0}\right\}$, and let $W_{m}$ be the lift of $w_{m}$ relative to the cube $Q_{r}\left(X_{0}, t_{0}\right)$. It is shown in [L] that $W_{m}$ is a subsolution.

Therefore we have that $v_{m} \leq w_{m} \leq W_{m} \leq u$ and hence $W_{m}\left(X_{0}, t_{0}+\frac{r}{8}\right) \rightarrow u\left(X_{0}, t_{0}+\frac{r}{8}\right)$ as $m \rightarrow \infty$.

By definition, we know that $W_{m}$ is a solution in $Q_{r}\left(X_{0}, t_{0}\right)$, and according to Nash [N], $W_{m}$ is Hölder continuous with bounds uniform in $m$, and therefore equicontinuous. As $v_{0} \leq W_{m} \leq u$, we see that $W_{m}$ is bounded, and therefore we can invoke Arzela-Ascoli to say that there exists a subsequence $\left\{W_{m_{k}}\right\}$ which converges uniformly in $Q_{\frac{r}{2}}\left(X_{0}, t_{0}\right)$ to a solution $w$ of $L w=0$ in $Q_{\frac{r}{2}}\left(X_{0}, t_{0}\right)$ with $w\left(X_{0}, t_{0}+\frac{r}{8}\right)=u\left(X_{0}, t_{0}+\frac{r}{8}\right)$.

If $\left(X_{1}, t_{1}\right)$ is any point in $Q_{\frac{r}{8}}\left(X_{0}, t_{0}\right)$, choose the sequence $\left\{v_{m}^{(1)}\right\}$ of subsolutions such that $v_{m}^{(1)}\left(X_{1}, t_{1}\right) \rightarrow u\left(X_{1}, t_{1}\right)$. Define $w_{m}^{(1)}=\max \left\{w_{m}, v_{m}^{(1)}\right\}$, and let $W_{m}^{(1)}$ be the lift of $w_{m}^{(1)}$ relative to the cube $Q_{\frac{r}{8}}\left(X_{0}, t_{0}\right)$. Then $W_{m}^{(1)}$ is a subsolution and we have that $v_{m}^{(1)} \leq w_{m}^{(1)} \leq W_{m}^{(1)} \leq u$ and $v_{m} \leq w_{m} \leq w_{m}^{(1)} \leq W_{m}^{(1)} \leq u$. Therefore, $W_{m}^{(1)}\left(X_{0}, t_{0}+\frac{r}{8}\right) \rightarrow u\left(X_{0}, t_{0}+\frac{r}{8}\right)$ and $W_{m}^{(1)}\left(X_{1}, t_{1}\right) \rightarrow$ $u\left(X_{1}, t_{1}\right)$.

Following the same process as above we can take a convergent subsequence of $W_{m}^{(1)}$ which converges uniformly in $Q_{\frac{r}{16}}\left(X_{0}, t_{0}\right)$ to a solution $w^{(1)}$ of $L w^{(1)}=0$ in $Q_{\frac{r}{16}}\left(\left(X_{0}, t_{0}\right)\right.$. We see that $w^{(1)} \geq w, w^{(1)}\left(X_{0}, t_{0}+\frac{r}{8}\right)=u\left(X_{0}, t_{0}+\frac{r}{8}\right)=w\left(X_{0}, t_{0}+\frac{r}{8}\right)$, and $w^{(1)}\left(X_{1}, t_{1}\right)=u\left(X_{1}, t_{1}\right)$. 
By the maximum principle we obtain that $w=w^{1}$ in $Q_{\frac{r}{8}}\left(X_{0}, t_{0}\right)$. Since $\left(X_{1}, t_{1}\right)$ was arbitrary, it follows that $w \equiv u$ in $Q_{\frac{r}{8}}\left(X_{0}, t_{0}\right)$. Hence $u$ is a solution of $L$ in a neighborhood of any point in $\Omega$ and therefore $L u=0$ in $\Omega$.

\subsubsection{Parabolic Bourgain-type Estimates}

In this section, we present a parabolic version of a fundamental result proved by Bourgain in the elliptic setting. Bourgain's Lemma [Bo] for elliptic measure has proved to be highly useful in numerous applications (e.g., see [ABHM], [HLe], [HMT]), and it seems reasonable to expect that the same will be true in the parabolic setting. In particular, we shall use the Bourgain-type lemma and its consequences in the proofs of our main results.

Let $a>0$ be the constant mentioned in Remark 1.2.25. In the sequel, $\Omega$ will always denote an open set in $\mathbb{R}^{n+1}$, with quasi-lateral boundary $\Sigma$. Given a fixed time $T<\infty$, we set

$$
E(T):=\left\{(X, t) \in \mathbb{R}^{n+1}: t<T\right\}
$$

Lemma 2.0.5 (Parabolic Bourgain-type Estimate). Let $\left(x_{0}, t_{0}\right) \in \Sigma$, and let $0<r<$ $\sqrt{t_{0}-T_{\min }} /(4 \sqrt{n})$. Assume that $\Sigma$ is time-backwards $A D R$ on $\Delta_{r}:=Q_{r}\left(x_{0}, t_{0}\right) \cap \Sigma$. Then there exists $M_{1}, \eta>0$ such that for all $(X, t) \in Q_{\frac{a}{M_{1}}} r \cap \Omega$,

$$
\omega^{X, t}\left(\Delta_{r}\right)=\omega^{X, t}\left(\Delta_{r} \cap E(T)\right) \geq \eta
$$

where $Q_{\frac{a}{M_{1}} r}:=Q\left(\left(x_{0}, t_{0}\right), \frac{a}{M_{1}} r\right)$, and $T:=T_{\max }\left(Q_{\frac{a}{M_{1}} r}\right)=t_{0}+\left(a M_{1}^{-1} r\right)^{2}$

Remark 2.0.6. This estimate could probably also be derived using capacitary methods found in [EG]. We give a direct proof adapting Bourgain's argument to the parabolic setting. 
Proof of Lemma 2.0.5. Let $\left(x_{0}, t_{0}\right) \in \Sigma$, and let $0<M r<\sqrt{t_{0}-T_{\min }} /(4 \sqrt{n})$, where our goal is now, equivalently, to show that $\omega^{X, t}\left(\Delta_{M r} \cap E(T)\right) \geq \eta$, for all $(X, t) \in Q_{\text {ar/2 }}\left(x_{0}, t_{0}\right) \cap \Omega$, with $\Sigma$ time-backwards ADR on $\Delta_{M r}=Q_{M r}\left(x_{0}, t_{0}\right) \cap \Sigma$, and $M$ is a large enough constant depending only on $\lambda, n$ and ADR (including time-backwards ADR). Here, $E(T)$ is defined as in (2.0.4), with $T=T_{\max }\left(Q_{a r / 2}\left(x_{0}, t_{0}\right)\right)=t_{0}+(a r)^{2} / 4$. We then obtain the conclusion of Lemma 2.0.5 with $M_{1}=2 M$.

We continue to assume either that $L$ is the heat operator, or that the continuous Dirichlet problem is solvable in $\Omega$ for $L$.

Claim 1. There exist numbers $a \in(0,1 / 2)$ and $b \in(0,1)$, depending only on $n$ and ADR (including the time-backwards ADR constants), such that if $\Sigma$ is time-backwards ADR on $\Delta=\Delta_{r}=\Sigma \cap Q_{r}\left(x_{0}, t_{0}\right)$, then

$$
\sigma\left(Q_{r}^{-}\left(x_{0}, t_{0}-(a r)^{2}\right) \cap \Sigma\right) \geq \sigma\left(\Delta_{r}^{-} \cap\left\{t<t_{0}-(a r)^{2}\right\}\right) \geq b r^{n+1}
$$

Proof of Claim 1. Observe that

$$
Q_{r}^{-}\left(x_{0}, t_{0}-(a r)^{2}\right) \supset Q_{r}^{-} \cap\left\{t<t_{0}-(a r)^{2}\right\}
$$

Thus, the first inequality in (2.0.7) is trivial, so we need only prove the second. Set $\Phi_{a r}:=$ $Q_{r}^{-}\left(x_{0}, t_{0}\right) \cap\left\{t_{0}-(a r)^{2} \leq t \leq t_{0}\right\}$ and take $a=2^{-m}$, where $m>1$ will be chosen large enough. We then decompose $\Phi_{a r}$ into sub-cubes, with parabolic length $a r$, all of equal size, and denote these cubes by $Q_{a r}^{i}$. As the $n$-dimensional measure of the face of each $Q_{a r}^{i}$ with $t=t_{0}$ is $(a r)^{n}$, and the $n$-dimensional measure of the face of $Q_{r}^{-}\left(x_{0}, t_{0}\right)$, again with $t=t_{0}$, is $r^{n}$, there are $a^{-n}$ such sub-cubes $Q_{a r}^{i}$. Hence,

$$
\Phi_{a r}=\bigcup_{i=1}^{a^{-n}} Q_{a r}^{i} .
$$


By upper ADR of $Q_{a r}^{i} \cap \Sigma$, this yields in turn that

$$
\sigma\left(\Sigma \cap \Phi_{a r}\right) \leq M_{0} \sum_{i=1}^{a^{-n}}(a r)^{n+1} \leq M_{0} a r^{n+1} .
$$

Therefore, if we choose $a \leq\left(2 M_{0}\right)^{-1} b_{0}$, where $b_{0}$ is the constant in the definition of timebackwards ADR,

$$
\sigma\left(\Sigma \cap \Phi_{a r}\right) \leq \frac{1}{2} b_{0} r^{n+1} \leq \frac{1}{2} \sigma\left(\Sigma \cap Q_{r}^{-}\left(x_{0}, t_{0}\right)\right)
$$

Consequently, since $Q_{r}^{-}\left(x_{0}, t_{0}\right) \backslash \Phi_{a r}=Q_{r}^{-} \cap\left\{t<t_{0}-(a r)^{2}\right\}$,

$$
\begin{aligned}
\sigma\left(\Delta_{r}^{-} \cap\left\{t<t_{0}-(a r)^{2}\right\}\right) & =\sigma\left(\Sigma \cap\left(Q_{r}^{-}\left(x_{0}, t_{0}\right) \backslash \Phi_{a r}\right)\right) \\
& \geq \frac{1}{2} \sigma\left(\Sigma \cap Q_{r}^{-}\left(x_{0}, t_{0}\right)\right) \geq \frac{1}{2} b_{0} r^{n+1},
\end{aligned}
$$

where we have used time-backwards ADR in the last step. This proves Claim 1 with $b:=\frac{1}{2} b_{0}$

Set

$$
\hat{\Delta}:=Q_{r}^{-}\left(x_{0}, t_{0}-(a r)^{2}\right) \cap \Sigma
$$

and define

$$
u(X, t):=\int_{-\infty}^{t} \int_{\Sigma_{s}} \Gamma(X, t, y, s) \chi_{\hat{\Delta}}(y, s) d \sigma_{s}(y) d s,
$$

where $\Gamma(X, t, y, s)$ is the fundamental solution of $L$. In $[\mathrm{QX}]$, the authors prove the following inequality:

$$
\begin{aligned}
\frac{1}{(N(t-s))^{n / 2}} \exp \left(-\frac{N|X-y|^{2}}{(t-s)}\right) \chi_{\{t>s\}} & \leq \Gamma(X, t, y, s) \\
& \leq \frac{N}{((t-s))^{n / 2}} \exp \left(-\frac{|X-y|^{2}}{N(t-s)}\right) \chi_{\{t>s\}},
\end{aligned}
$$

where $N$ depends on dimension and $\lambda$.

Remark 2.0.12. In fact, in $[\mathrm{QX}]$, the authors obtain this inequality in the more general situation that the non-symmetric part of the coeffcient matrix $A$ belongs to $B M O$. 
Claim 2.0.13. The function $u$ defined in (2.0.10) satisfies the following properties: there exist constants $C_{1}$ and $c_{2}$ depending only on $\mathrm{ADR}, \lambda$, and $n$, such that

(1) $u$ is continuous in $\mathbb{R}^{n+1}, L u=0$ in $\Omega$, and $u \equiv 0$ for $\left\{t: t \leq t_{0}-\frac{5}{4} r^{2}\right\}$.

(2) $0 \leq u \leq C_{1} r$ in $\mathbb{R}^{n+1}$.

(3) $u(X, t) \geq c_{2} r$, for $(X, t) \in Q_{a r / 2} \cap \bar{\Omega}$, where $Q_{a r / 2}:=Q_{a r / 2}\left(x_{0}, t_{0}\right)$.

(4) $u(X, t) \leq C_{1} M^{-n} r$, for $(X, t) \in \bar{\Omega} \backslash Q_{M r / 2}$, where $Q_{M r / 2}:=Q\left(\left(x_{0}, t_{0}\right), \frac{M}{2} r\right)$.

Property (1) follows by definition of $u$, and the fact that $(a r)^{2} \leq r^{2} / 4$. Let us now verify the remaining properties.

Proof of property (2). Certainly, $u \geq 0$, by definition. To prove the upper bound for $u$, we first prove the following claim.

\section{Claim 2.}

$$
\Gamma(X, t, y, s) \lessgtr_{n, \lambda}\|X-y, t-s\|^{-n},
$$

Proof of Claim 2. We consider two cases.

Case 1. $|X-y|<(t-s)^{1 / 2}$

Then $\|X-y, t-s\| \approx(t-s)^{1 / 2}$, and hence

$$
\begin{aligned}
\Gamma(X, t, y, s) & \leq \frac{N}{((t-s))^{n / 2}} \exp \left(-\frac{|X-y|^{2}}{N(t-s)}\right) \chi_{\{t>s\}} \\
& \leq \frac{N}{((t-s))^{n / 2}} \\
& \approx \frac{1}{\left((t-s)^{1 / 2}\right)^{n}} \\
& \approx\|X-y, t-s\|^{-n} .
\end{aligned}
$$


Case 2. $(t-s)^{1 / 2} \leq|X-y|$

Then $\|X-y, t-s\| \approx|X-y|$, and hence

$$
\begin{aligned}
\Gamma(X, t, y, s) & \leq \frac{N}{((t-s))^{n / 2}} \exp \left(-\frac{|X-y|^{2}}{N(t-s)}\right) \chi_{\{t>s\}} \\
& =\frac{|X-y|^{n}}{((t-s))^{n / 2}} \exp \left(-\frac{|X-y|^{2}}{N(t-s)}\right) \frac{N}{|X-y|^{n}} \chi_{\{t>s\}} \\
& \lesssim \frac{N}{|X-y|^{n}} \\
& \approx\|X-y, t-s\|^{-n}
\end{aligned}
$$

using the fact that $\exp (-a) a^{n} \leq c_{n}$.

Next, we split $u$ as follows:

$$
\begin{aligned}
u(X, t) & =\iint_{\hat{\Delta}} \Gamma(X, t, y, s) d \sigma(y, s) \\
& =\iint_{\hat{\Delta} \cap\{\|X-y, t-s\|<r\}} \Gamma d \sigma+\iint_{\hat{\Delta} \cap\{\|X-y, t-s\| \geq r\}} \Gamma d \sigma=: I+I I .
\end{aligned}
$$

By (2.0.14), the integrand in term $I I$ at most $C r^{-n}$, hence,

$$
I I \lesssim r^{-n} \sigma(\hat{\Delta}) \lesssim r^{-n} r^{n+1} \leq C r
$$

by upper $\mathrm{ADR}$, where $C=C(\mathrm{ADR}, n, \lambda)$.

In term $I$, we will dyadically decompose $\widetilde{\Delta}:=\hat{\Delta} \cap\{\|X-y, t-s\|<r\}$. Define

$$
A_{k}:=\left\{(y, s) \in \widetilde{\Delta}: 2^{-k-1} r<\|X-y, t-s\| \leq 2^{-k} r\right\}
$$

As $A_{k} \subset \hat{\Delta}$, we can use upper ADR on $A_{k}$, along with (2.0.14), to obtain the following estimate:

$$
\begin{aligned}
I & \lesssim \sum_{k=0}^{\infty} \iint_{A_{k}}\|X-y, t-s\|^{-n} d \sigma(y, s) \\
& \leq \sum_{k=0}^{\infty}\left(2^{-k-1} r\right)^{-n} \iint_{A_{k}} d \sigma(y, s)
\end{aligned}
$$




$$
\lesssim \sum_{k=0}^{\infty}\left(2^{-k-1} r\right)^{-n}\left(2^{-k} r\right)^{n+1} \leq C r
$$

where $C=C(\mathrm{ADR}, n, \lambda)$.

Proof of property (3). As $(y, s) \in \hat{\Delta}$ and $(X, t) \in Q_{a r / 2} \cap \bar{\Omega}$, we have that

$$
\begin{gathered}
t_{0}-(a r)^{2}-r^{2}<s<t_{0}-(a r)^{2}, \\
t_{0}-\frac{1}{4}(a r)^{2}<t<t_{0}+\frac{1}{4}(a r)^{2} .
\end{gathered}
$$

Hence

$$
\begin{aligned}
& t-s<t_{0}+\frac{1}{4}(a r)^{2}-\left(t_{0}-(a r)^{2}-r^{2}\right)=\frac{5}{4}(a r)^{2}+r^{2}, \quad \text { and } \\
& t-s>t_{0}-\frac{1}{4}(a r)^{2}-\left(t_{0}-(a r)^{2}\right)=\frac{3}{4}(a r)^{2} .
\end{aligned}
$$

Overall, this gives us that $t-s \approx r^{2}$, with implicit constants depending on $a$. Since $\left|X-x_{0}\right| \lesssim$ $r$ and $\left|y-x_{0}\right| \lesssim r$, by the triangle inequality we have $|X-y| \lesssim r$, hence,

$$
-|X-y|^{2} \gtrsim-r^{2}
$$

Combining the above estimates, as well as using ADR, we obtain that

$$
\begin{aligned}
u(X, t) & \geq \iint_{\hat{\Delta}} \frac{1}{(N(t-s))^{n / 2}} \exp \left(-\frac{N|X-y|^{2}}{(t-s)}\right) \chi_{\{t>s\}} d \sigma(y, s) \\
& \gtrsim \iint_{\hat{\Delta}} \frac{1}{r^{n}} \exp \left(-\frac{r^{2}}{C r^{2}}\right) d \sigma(y, s) \\
& \gtrsim r^{-n} \sigma(\hat{\Delta}) \gtrsim r^{-n} r^{n+1} \geq c_{2} r,
\end{aligned}
$$

by (2.0.7) and the definition of $\hat{\Delta}(2.0 .9)$, where $c_{2}=c_{2}(n, A D R, \lambda)>0$ (recall that by Claim 1, $a$ depends only on $n$, ADR and time-backwards ADR).

Proof of property (4). As $(y, s) \in \hat{\Delta}$ and $(X, t) \in \bar{\Omega} \backslash Q_{M r / 2}$, we have that

$$
\|(X, t)-(y, s)\| \geq\left|\left\|(X, t)-\left(x_{0}, t_{0}\right)\right\|-\left\|\left(x_{0}, t_{0}\right)-(y, s)\right\|\right| \geq|c M r-C r| \gtrsim M r,
$$


provided that $M$ is chosen sufficiently large. Combining the latter estimate with $(2.0 .14)$ and (2.0.7), we have

$$
u(X, t) \lesssim_{n, \lambda}(M r)^{-n} \sigma(\hat{\Delta}) \leq C_{1} M^{-n} r
$$

where $C_{1}=C_{1}(n, A D R, \lambda)$.

Claim 2.0.15. Set $\tilde{u}(X, t):=\frac{1}{r}\left(u(X, t)-\sup _{\bar{\Omega} \backslash Q_{M r / 2}} u\right)$. Then $\tilde{u}$ satisfies the following:

(i) $\tilde{u}$ is continuous in $\mathbb{R}^{n+1}, L \tilde{u}=0$ in $\Omega$, and $\tilde{u} \leq 0$ in $\bar{\Omega} \backslash Q_{M r / 2}$, and in $\left\{t: t \leq t_{0}-\frac{5}{4} r^{2}\right\}$.

(ii) $|\tilde{u}(X, t)| \leq C_{1}$ in $\bar{\Omega}$.

(iii) $\tilde{u}(X, t) \geq \frac{1}{2} c_{2}$ for $(X, t) \in Q_{a r / 2} \cap \bar{\Omega}$, provided $M$ is large enough.

Property (i) follows immediately from Claim 2.0.13, property (1), and the definition of $\tilde{u}$.

Proof of property (ii). Since $0 \leq u \leq C_{1} r$ in $\mathbb{R}^{n+1}$, for $(X, t) \in \bar{\Omega}$,

$$
\begin{aligned}
|\tilde{u}(X, t)| & =\left|\frac{1}{r}\left(u(X, t)-\sup _{\bar{\Omega} \backslash Q_{M r / 2}} u\right)\right| \\
& \leq \frac{1}{r} u(X, t) \leq \frac{1}{r} C_{1} r=C_{1} .
\end{aligned}
$$

Proof of property (iii). Applying properties (3) and then (4) from Claim 2.0.13, we obtain that for $(X, t) \in Q_{a r / 2} \cap \bar{\Omega}$

$$
\begin{aligned}
\tilde{u}(X, t) & =\frac{1}{r}\left(u(X, t)-\sup _{\bar{\Omega} \backslash Q_{M r / 2}} u\right) \\
& \geq c_{2}-\frac{1}{r} \sup _{\bar{\Omega} \backslash Q_{M r / 2}} u \\
& \geq c_{2}-C_{1} M^{-n} \geq \frac{1}{2} c_{2},
\end{aligned}
$$

choosing $M$ large enough so that $C_{1} M^{-n} \leq \frac{1}{2} c_{2}$. 
Recall that $E(T)$ is defined as in (2.0.4), with $T:=T_{\max }\left(Q_{a r / 2}\right)=t_{0}+(a r)^{2} / 4$.

Claim 3. $\omega^{X, t}\left(\Delta_{M r} \cap E(T)\right) \gtrsim \tilde{u}(X, t)$, for

$$
(X, t) \in \widetilde{\Omega}:=\Omega \cap Q_{M r / 2} \cap\left\{t: t>t_{0}-5 r^{2} / 4\right\} \cap E(T) .
$$

Proof of Claim 3. Let $0<\varepsilon \ll r$. Set $\widetilde{\Omega}_{\varepsilon}:=\widetilde{\Omega} \cap E(T-\varepsilon)$. Observe that by property (i) and the definition of $\widetilde{\Omega}_{\varepsilon}$,

$$
\mathcal{P} \widetilde{\Omega}_{\varepsilon} \subset\{\tilde{u} \leq 0\} \cup\left(\overline{Q_{M r / 2}} \cap \Sigma \cap E(T)\right) \subset\{\tilde{u} \leq 0\} \cup\left(\Delta_{M r} \cap E(T)\right)
$$

The claim then follows with $\widetilde{\Omega}_{\varepsilon}$ in place of $\widetilde{\Omega}$, by property (ii) and the weak maximum principle. The full claim follows by letting $\varepsilon \rightarrow 0$.

Note that $Q_{a r / 2} \cap \Omega \subset \widetilde{\Omega}$, since $t_{0}-(a r)^{2} / 4<t<T$ in $Q_{a r / 2}$. By Claim 2.0.15, property (iii), we have that $\tilde{u}(X, t) \geq \frac{1}{2} c_{2}$ for $(X, t) \in Q_{a r / 2} \cap \Omega$. Thus, for such $(X, t)$, by Claim 3 we obtain

$$
\frac{1}{2} c_{2} \leq \tilde{u}(X, t) \lesssim \omega^{X, t}\left(\Delta_{M r}\right)
$$

This finishes the proof with $\eta \approx c_{2}$.

Remark 2.0.16. One may readily deduce the following consequence of Lemma 2.0.5. Let $\Sigma$ be globally TBADR. Then there is a constant $M_{2} \approx_{n} M_{1} / a$, such that, given $(X, t) \in \Omega$, with $\left.2 M_{2} \delta_{\infty}(X, t)<\min \left(R_{0}, \sqrt{t-T_{\min }}\right)\right)$, if $(\hat{x}, \hat{t}) \in \Sigma$ is a touching point for $(X, t)$, so that $\|(X, t)-(\hat{x}, \hat{t})\|_{\ell^{\infty}}=\delta_{\infty}(X, t)=: r$, and if

$$
\Delta_{X, t}:=\Delta\left((\hat{x}, \hat{t}), M_{2} r\right)=\Sigma \cap Q\left((\hat{x}, \hat{t}), M_{2} r\right)
$$

then

$$
\omega^{X, t}\left(\Delta_{X, t}\right) \geq \kappa
$$


Given a fixed time $T$, and a cube $Q_{r}$ centered on $\Sigma$, we set $\Omega_{r}:=Q_{r} \cap \Omega$, and $\Omega_{r}(T):=$ $\Omega_{r} \cap E(T)$, with $E(T)$ defined as in (2.0.4).

Lemma 2.0.19 (Parabolic Bourgain-type Estimate for supersolutions). Let $\left(x_{0}, t_{0}\right) \in \Sigma$, and let $0<r<\sqrt{t_{0}-T_{\min }} /(4 \sqrt{n})$. Set $Q_{r}:=Q_{r}\left(x_{0}, t_{0}\right), Q_{a r / M_{1}}=Q\left(\left(x_{0}, t_{0}\right), \frac{a}{M_{1}} r\right)$, and define

$$
T=T_{\max }\left(Q_{a r / M_{1}}\right)=t_{0}+\left(a M_{1}^{-1} r\right)^{2} .
$$

Assume that $\Sigma$ is time-backwards ADR on $\Delta_{r}:=Q_{r} \cap \Sigma$. Then there exists $M_{1}, \eta>0$ such that if $w$ is a non-negative supersolution in $\Omega_{r}(T)$, with $w \geq 1$ on $\Delta_{r} \cap E(T)$ in the sense that

$$
\liminf _{(X, t) \rightarrow(y, s)} w(X, t) \geq 1, \quad(y, s) \in \Delta_{r} \cap E(T),
$$

then

$$
w(X, t) \geq \eta, \quad \forall(X, t) \in \Omega_{a r / M_{1}}
$$

Proof. Let $\left(x_{0}, t_{0}\right) \in \Sigma$, and let $0<M r<\sqrt{t_{0}-T_{\min }} /(4 \sqrt{n})$, where our goal is now, equivalently, to show that $w(X, t) \geq \eta$, for all $(X, t) \in Q_{a r}\left(x_{0}, t_{0}\right) \cap \Omega$, assuming $w \geq 1$ on $\Delta_{M r} \cap E\left(T^{\prime}\right), T^{\prime}=t_{0}+(a r)^{2}$, with $\Sigma$ time-backwards ADR on $\Delta_{M r}$, and $M=M_{1}$ is a large enough constant depending only on $\lambda, n$ and ADR (including time-backwards ADR).

We then follow the construction as in Lemma 2.0.5, first building the same auxiliary solutions $u$ and $\tilde{u}$.

By construction we have that

$$
L w \leq L \tilde{u} \quad \text { in } \Omega .
$$

Since $w \geq 1$ on $\Delta_{M r} \cap E\left(T^{\prime}\right)$ and $\tilde{u} \lesssim 1$ we obtain that $\tilde{u} \lesssim w$ in $\Delta_{M r} \cap E\left(T^{\prime}\right)$.

By the weak minimum principle for supersolutions we then obtain that

$$
w \geq c \tilde{u} \quad \text { in } \Omega \cap Q_{M r} \cap E\left(T^{\prime}\right) .
$$


Combining the above estimate, as well as Claim 2.0.15 (iii), we obtain that

$$
w(X, t) \geq c \tilde{u}(X, t) \gtrsim \frac{1}{2} c c_{2}:=\eta
$$

for $(X, t) \in Q_{a r} \cap \Omega$.

\subsubsection{Hölder Continuity at the Boundary}

The following lemmas are consequences of Lemma 2.0.19. We first state the lemma for subsolutions.

Lemma 2.0.20. Suppose that $\Sigma$ is time-backwards $A D R$ on $Q_{2 r}:=Q_{2 r}\left(x_{0}, t_{0}\right)$, with $\left(x_{0}, t_{0}\right) \in$ $\Sigma$ and $0<r<\sqrt{t_{0}-T_{\min }} /(8 \sqrt{n})$. Then there exists $C=C(n, A D R), \alpha=\alpha(n, A D R)>0$, such that if $v$ is a non-negative subsolution in $\Omega_{2 r}\left(T_{1}\right)$, which vanishes continuously on $\Delta_{2 r} \cap E\left(T_{1}\right):=Q_{2 r} \cap \Sigma \cap E\left(T_{1}\right)$, then

$$
v(Y, t) \leq C\left(\frac{\delta(Y, t)}{r}\right)^{\alpha} \mathcal{M}(v), \quad \forall(Y, t) \in \Omega_{r},
$$

where $T_{1}:=T_{\max }\left(Q_{r}\right)=t_{0}+r^{2}$, and $\mathcal{M}(v):=\sup _{\Omega_{3 r / 2}\left(T_{1}\right)} v$.

Proof of Lemma 2.0.20. If $\mathcal{M}(v)=\infty$, then there is nothing to prove, so we may assume that $\mathcal{M}(v)<\infty$. Normalize $v$ so that $\mathcal{M}(v) \leq 1$, and set $w:=1-v$. Then $0 \leq w \leq 1$ in $\Omega_{3 r / 2}\left(T_{1}\right)$, and $w \equiv 1$ on $\Delta_{2 r} \cap E\left(T_{1}\right)$. Then by Lemma 2.0 .19 , there exists $M_{1}, \eta>0$ such that $w(Y, t) \geq \eta$, and therefore

$$
v(Y, t) \leq 1-\eta, \quad \forall(Y, t) \in Q_{\frac{a}{M_{1}}} r\left(x_{0}, t_{0}\right) \cap \Omega
$$

Iterating, we obtain

$$
v(Y, t) \leq(1-\eta)^{k}:=\theta_{0}^{k}, \quad \forall(Y, t) \in Q_{\frac{a^{k}}{M_{1}^{k}} r}\left(x_{0}, t_{0}\right) \cap \Omega
$$


Choose $\alpha$ such that $\alpha=-\log \theta_{0}\left(\log M_{1}\right)^{-1}$. Hence $\theta_{0}=M_{1}^{-\alpha}$, and

$$
v(Y, t) \leq M_{1}^{-\alpha k}, \quad \forall(Y, t) \in Q_{\frac{a^{k}}{M_{1}^{k}} r}\left(x_{0}, t_{0}\right) \cap \Omega
$$

As $\delta(Y, t) \approx r M_{1}^{-k}$, we have that $M_{1}^{-k} \approx \delta(Y, t) r^{-1}$, and therefore,

$$
v(Y, t) \leq C\left(\frac{\delta(Y, t)}{r}\right)^{\alpha}, \quad \forall(Y, t) \in Q_{\frac{a^{k}}{M_{1}^{k}}}\left(x_{0}, t_{0}\right) \cap \Omega .
$$

Lemma 2.0.21. Let $\left(x_{0}, t_{0}\right) \in \Sigma$, and fix $r$ with $0<r<\sqrt{t_{0}-T_{\min }} /(8 \sqrt{n})$. Set $Q_{r}:=$ $Q_{r}\left(x_{0}, t_{0}\right), Q_{2 r}:=Q_{2 r}\left(x_{0}, t_{0}\right)$, and suppose that $\Sigma$ is time-backwards ADR on $\Delta_{2 r}:=Q_{2 r} \cap \Sigma$. Let $u$ be the parabolic measure solution corresponding to non-negative data $f \in C_{c}\left(\partial_{e} \Omega\right)$, with $f \equiv 0$ on $\Delta_{2 r}$. Then for some $\alpha>0$,

$$
u(Y, t) \leq C\left(\frac{\delta(Y, t)}{r}\right)^{\alpha} \frac{1}{\left|Q_{2 r} \cap E\left(T_{1}\right)\right|} \iint_{\Omega_{2 r}\left(T_{1}\right)} u, \quad \forall(Y, t) \in \Omega_{r}
$$

where $T_{1}:=T_{\max }\left(Q_{r}\right)=t_{0}+r^{2}$, and where the constants $C$ and $\alpha$ depend only on $n, \lambda$, and the ADR and time-backwards ADR constants.

We observe that in this paper it will suffice to have a slightly less sharp version of Lemma 2.0.21, in which $\Omega_{2 r}\left(T_{1}\right)$ is replaced by the larger set $\Omega_{2 r}$; see the proof of Theorem 3.2.1 below.

Proof. Set

$$
T_{1}:=T_{\max }\left(Q_{r}\left(x_{0}, t_{0}\right)\right)=t_{0}+r^{2}
$$

Set $\mathcal{M}(u):=\sup _{\Omega_{3 r / 2}\left(T_{1}\right)} u$. The first step is to establish the estimate

$$
u(Y, t) \leq C\left(\frac{\delta(Y, t)}{r}\right)^{\alpha} \mathcal{M}(u), \quad \forall(Y, t) \in \Omega_{r}:=Q_{r} \cap \Omega
$$


where $u$ is the parabolic measure solution with non-negative data $f \in C_{c}\left(\partial_{e} \Omega\right)$, with $f \equiv 0$ on $\Delta_{2 r}$. If $L$ is a divergence form parabolic operator for which the continuous Dirichlet problem is solvable, then estimate (2.0.22) is a special case of Lemma 2.0.20, since a nonnegative solution is in particular a non-negative subsolution.

On the other hand, suppose now that $L$ is the heat operator. In this case, we need not assume a priori solvability of the continuous Dirichlet problem; rather we shall use Lemma 2.0.20, and the Perron construction (see [W2, Chapter 8]). By resolutivity of $C\left(\partial_{e} \Omega\right.$ ), the caloric measure solution is the Perron (or PWB) solution, and is given by

$$
u=\sup \left\{v: v \in \mathcal{L}_{f}\right\}
$$

where, since $f \geq 0$, without loss of generality the lower class $\mathcal{L}_{f}$ consists of all nonnegative subcaloric $v$ satisfying

$$
\limsup _{(X, t) \rightarrow(y, s)} v(X, t) \leq f(y, s), \quad(y, s) \in \partial_{n} \Omega,
$$

and

$$
\limsup _{(X, t) \rightarrow\left(y, s^{+}\right)} v(X, t) \leq f(y, s), \quad(y, s) \in \partial_{s s} \Omega .
$$

In particular, since $\Delta_{2 r} \subset \Sigma \subset \partial_{n} \Omega$ (recall that $\partial_{s s} \Omega \cap \Delta_{2 r}=\emptyset$, by the time-backwards ADR assumption; see Remark 1.2.23), we have

$$
0 \leq \limsup _{(X, t) \rightarrow(y, s)} v(X, t) \leq f(y, s)=0, \quad(y, s) \in \Delta_{2 r},
$$

for all $v \in \mathcal{L}_{f}$. Thus, each such $v$ vanishes continuously on $\Delta_{2 r}$, and therefore Lemma 2.0.20 may be applied to any $v \in \mathcal{L}_{f}$.

If $\mathcal{M}(u)=\infty$, then (2.0.22) is trivial, so after normalizing, we may suppose that $\mathcal{M}(u) \leq$ 1. By the PWB construction, we then have $\mathcal{M}(v):=\sup _{\Omega_{3 r / 2}\left(T_{1}\right)} v \leq 1$, for all $v \in \mathcal{L}_{f}$. Given 
$\varepsilon>0$, and a point $(Y, t) \in \Omega_{r}$, we may choose $v \in \mathcal{L}_{f}$ such that

$$
u(Y, t) \leq v(Y, t)+\varepsilon
$$

Applying Lemma 2.0.20 to $v$, with $\mathcal{M}(v) \leq 1$, and letting $\varepsilon \rightarrow 0$, we obtain (2.0.22).

With (2.0.22) in hand, it remains to replace $\mathcal{M}(u)$ by an integral average. To this end, we first observe that $u$ vanishes continuously on $\Delta_{2 r}$. Indeed, in the case that the continuous Dirichlet problem is solvable for $L$, this fact holds by assumption. On the other hand, in the case that $L$ is the heat operator, we may use the time-backwards ADR assumption (and the Wiener-type criterion of [EG]) to deduce that every point in $\Delta_{2 r}$ is regular (see Lemma 2.0.2). Thus, in either case, $u$ vanishes continuously on $\Delta_{2 r}$. We may then extend $u \equiv 0$ in $Q_{2 r} \backslash \bar{\Omega}$, and we call this extension $\hat{u}$. Observe that $\hat{u} \geq 0$ and $\hat{u}$ is a subsolution in $Q_{2 r}$. Therefore, by local boundedness [M, Theorem 3], recalling that $T=T_{\max }\left(Q_{r}\right)=t_{0}+r^{2}$, we obtain

$$
\mathcal{M}(u)=\sup _{Q_{3 r / 2} \cap E(T)} \hat{u} \lesssim \mathcal{f}_{Q_{2 r} \cap E(T)} \hat{u}=C r^{-n-2} \iint_{\Omega_{2 r}(T)} u .
$$

We note that Theorem 3 in $[\mathrm{M}]$ is stated with an $L^{p}$ average, $p \geq 2$, on the right hand side of the inequality, but in hindsight, this may be sharpened to an $L^{1}$ average, using a well-known self-improving property of weak reverse Hölder estimates.

\subsubsection{Boundary Harnack}

Lemma 2.0.24 (Boundary Harnack Estimate). Let $\Omega \subset \mathbb{R}^{n+1} \cap\{t>0\}$ be a bounded domain with lateral boundary $\Sigma$, and let $L$ be defined as above. Assume $\Omega$ satisfies the Harnack Chain condition, interior corkscrews, and $\Sigma$ is time-symmetric ADR. Let $\left(x_{0}, t_{0}\right) \in \Sigma$ and fix $r$ with $0<r \leq \frac{\sqrt{T_{\max }-t_{0}}}{2}$. Let $u \in W_{2}^{1,0}\left(Q_{2 r}\left(x_{0}, t_{0}\right) \cap \Omega\right)$ be a non-negative solution of $L u=0$ 
in $Q_{2 r}\left(x_{0}, t_{0}\right) \cap \Omega$, which vanishes continuously on $\Delta_{2 r}:=Q_{2 r}\left(x_{0}, t_{0}\right) \cap \Sigma$. Then

$$
\sup _{Q_{r}\left(x_{0}, t_{0}\right) \cap \Omega} u(Y, s) \leq C u\left(X_{Q}, t_{Q}\right)
$$

where $\delta\left(X_{Q}, t_{Q}\right) \gtrsim r, t_{Q}:=t_{0}+\frac{3}{2} r^{2}$, and $\operatorname{dist}\left(\left(X_{Q}, t_{Q}\right), Q_{r}\left(x_{0}, t_{0}\right)\right) \lesssim r$. We call $\left(X_{Q}, t_{Q}\right) a$ corkscrew point relative to $Q:=Q_{r}\left(x_{0}, t_{0}\right)$.

To prove the Boundary Harnack estimate we need the following lemma, which guarantees the existence of time-forward interior corkscrews in the setting of Lemma 2.0.24.

Lemma 2.0.26. Let $\Omega \subset \mathbb{R}^{n+1} \cap\{t>0\}$ be a domain with lateral boundary $\Sigma$. Assume that $\Omega$ satisfies the Harnack Chain condition, has interior corkscrews, and $\Sigma$ is time-symmetric ADR. Then there exists time-forward interior corkscrews for all time values.

Proof. Let $\left(x_{0}, t_{0}\right) \in \Sigma$ and consider $Q_{r}\left(x_{0}, t_{0}\right), 0<r<\sqrt{t_{0}} / 2$. Since $\Omega$ has interior corkscrews, there must exist some point $(Y, s) \in Q_{r}\left(x_{0}, t_{0}\right) \cap \Omega$ such that $\delta(Y, s) \geq \zeta^{-1} r$. As $\Sigma$ is time-symmetric ADR and $\left(x_{0}, t_{0}\right) \in \Sigma$, there exists $(\tilde{x}, \tilde{t}) \in \Sigma$ such that $\left\|\left(x_{0}, t_{0}\right)-(\tilde{x}, \tilde{t})\right\| \leq$ $\mathrm{Cr}$ and

$$
t_{0}+25 r^{2}<\tilde{t}
$$

Then we can consider an interior corkscrew point relative to $Q_{r}(\tilde{x}, \tilde{t})$. In other words, there exists some $(\tilde{Y}, \tilde{s}) \in Q_{r}(\tilde{x}, \tilde{t}) \cap \Omega$ such that $\delta(\tilde{Y}, \tilde{s}) \geq \zeta^{-1} r$ and $\tilde{s}-\tilde{t} \approx \zeta r^{2}$. As $\delta(Y, s), \delta(\tilde{Y}, \tilde{s}) \geq$ $\zeta^{-1} r,\|(Y, s)-(\tilde{Y}, \tilde{s})\| \leq C r$, and $s+16 r^{2}<\tilde{s}$, we can construct a Harnack path, moving forward in time, connecting $(Y, s)$ to $(\tilde{Y}, \tilde{s})$ as $\Omega$ satifies the Harnack Chain condition. Any intermediate point in the Harnack path would still be an interior corkscrew point.

Remark 2.0.27. Lemma 2.0.26 guarantees such a point $\left(X_{Q}, t_{Q}\right)$ in Lemma 2.0.24 to exist.

Proof of Lemma 2.0.24. Define 


$$
\tilde{u}= \begin{cases}u & \text { in } \Omega \cap Q_{2 r}\left(x_{0}, t_{0}\right) \\ 0 & \text { in } Q_{2 r}\left(x_{0}, t_{0}\right) \backslash \Omega\end{cases}
$$

Then $\tilde{u}$ is a non-negative subsolution of $L$ in $Q_{2 r}\left(x_{0}, t_{0}\right)$ and therefore by Moser [M] (Theorem 3), we obtain that

$$
\begin{aligned}
\sup _{Q_{r}\left(x_{0}, t_{0}\right) \cap \Omega} u(Y, s) & =\sup _{Q_{r}\left(x_{0}, t_{0}\right) \cap \Omega} \tilde{u}(Y, s) \lesssim\left(\frac{1}{\left|Q_{2 r}\left(x_{0}, t_{0}\right)\right|} \iint_{Q_{2 r}\left(x_{0}, t_{0}\right) \cap \Omega}|\tilde{u}(Y, s)|^{2} d Y d s\right)^{1 / 2} \\
& =\left(\frac{1}{\left|Q_{2 r}\left(x_{0}, t_{0}\right)\right|} \iint_{Q_{2 r}\left(x_{0}, t_{0}\right) \cap \Omega}|u(Y, s)|^{2} d Y d s\right)^{1 / 2} \\
& <\infty
\end{aligned}
$$

since $u \in W_{2}^{1,0}\left(Q_{2 r}\left(x_{0}, t_{0}\right) \cap \Omega\right)$.

Claim 1. It is enough to assume that $u$ vanishes continuously on $\Delta_{2 C_{n} r}$.

Proof of Claim 1. For $\epsilon>0$ define $\widetilde{\Delta}:=\Delta_{(1+\epsilon) r}\left(x_{0}, t_{0}\right)$, and write

$$
\widetilde{\Delta} \subset \bigcup_{(x, t) \in \widetilde{\Delta}} \Delta_{r^{\prime}}(x, t)
$$

where $10 C_{n} r^{\prime}=r$ for some $C_{n} \geq 1$. Then for every $\Delta_{r^{\prime}}(x, t), \Delta_{2 C_{n} r^{\prime}}(x, t) \subset \Delta_{2 r}$ and therefore if $u$ vanishes continuously on $\Delta_{2 r}$, we must have that $u$ vanishes continuously on $\Delta_{2 C_{n} r^{\prime}}(x, t)$.

Suppose that (2.0.25) holds for $u$ in $Q_{r^{\prime}}(x, t)$, where $u$ vanishes continuously on $\Delta_{2 C_{n} r^{\prime}}(x, t)$. Then

$$
\sup _{Q_{r^{\prime}}(x, t) \cap \Omega} u(Y, s) \leq C u\left(X_{Q^{\prime}}, t_{Q^{\prime}}\right)
$$

where $Q^{\prime}:=Q_{r^{\prime}}(x, t)$ and $\left(X_{Q^{\prime}}, t_{Q^{\prime}}\right)$ is such that $t_{Q^{\prime}}=t+\frac{3}{2} r^{\prime 2}, \delta\left(X_{Q^{\prime}}, t_{Q^{\prime}}\right) \gtrsim r^{\prime}$, and $\operatorname{dist}\left(\left(X_{Q^{\prime}}, t_{Q^{\prime}}\right), Q^{\prime}\right) \lesssim r^{\prime}$. Then

$$
t_{Q^{\prime}}:=t+\frac{3}{2} r^{\prime 2}<t_{0}+\frac{5}{4} r^{2}=t_{Q}-\frac{1}{4} r^{2}
$$

or equivalently,

$$
t_{Q^{\prime}}+\frac{1}{4} r^{2}<t_{Q}
$$


Therefore by Harnack, we have that

$$
u\left(X_{Q^{\prime}}, t_{Q^{\prime}}\right) \lesssim u\left(X_{Q}, t_{Q}\right)
$$

For $(Y, s) \in Q_{r}\left(x_{0}, t_{0}\right) \backslash\left(\bigcup_{(x, t) \in \widetilde{\Delta}} Q_{r^{\prime}}(x, t)\right)$ we have that

$$
\delta(Y, s) \gtrsim_{\epsilon} r
$$

Then as $\Omega$ satisfies the Harnack Chain condition and $\delta(Y, s), \delta\left(X_{Q}, t_{Q}\right) \gtrsim r, s+\frac{1}{2} r^{2} \leq t_{Q}$, we obtain that

$$
u(Y, s) \leq C u\left(X_{Q}, t_{Q}\right)
$$

We will now assume that $u$ vanishes continuously on $\Delta_{2 C_{n} r}$.

Without loss of generality we will assume that $u\left(X_{Q}, t_{Q}\right)=1$. We want to show for all $(Y, s) \in Q_{r}\left(x_{0}, t_{0}\right)$

$$
u(Y, s) \leq C u\left(X_{Q}, t_{Q}\right)=C
$$

We will prove this by contradiction. Suppose $\exists\left(Y_{0}, s_{0}\right) \in Q_{r}\left(x_{0}, t_{0}\right)$ such that

$$
u\left(Y_{0}, s_{0}\right) \geq C_{0}^{N}
$$

for $C_{0}$ sufficiently large and some $N$ to be chosen.

Claim 2. $u\left(Y_{0}, s_{0}\right) \geq C_{0}^{N}$ implies $\delta\left(Y_{0}, s_{0}\right) \leq 2^{-N} r$.

Proof of Claim 2. We will prove this by contradiction. Suppose $u\left(Y_{0}, s_{0}\right) \geq C_{0}^{N}$ and $\delta\left(Y_{0}, s_{0}\right)>$ $2^{-N} r$. Then as $\delta\left(X_{Q}, t_{Q}\right), \delta\left(Y_{0}, s_{0}\right)>2^{-N} r$, for $N$ large enough, and $s_{0}+\frac{1}{2} r^{2} \leq t_{Q}$ we have by the Harnack Chain condition that $\left(Y_{0}, s_{0}\right)$ can be connected by a chain of Whitney cubes to $\left(X_{Q}, t_{Q}\right)$, of cardinality at most

$$
C(\gamma) \log \left(2+\frac{\left\|\left(X_{Q}, t_{Q}\right)-\left(Y_{0}, s_{0}\right)\right\|}{\min \left\{\delta\left(X_{Q}, t_{Q}\right), \delta\left(Y_{0}, s_{0}\right)\right\}}\right) \lesssim C(\gamma) \log \left(2+\frac{r}{2^{-N} r}\right)
$$




$$
\begin{aligned}
& =C(\gamma) \log \left(2+2^{N}\right) \\
& \leq C N .
\end{aligned}
$$

Therefore, using Harnack, we have that

$$
C_{0}^{N} \leq u\left(Y_{0}, s_{0}\right) \leq C(\gamma)^{C N} u\left(X_{Q}, t_{Q}\right)=C^{N}
$$

However, this is a contradiction, for $N$ chosen large enough.

Hence as $u\left(Y_{0}, s_{0}\right) \geq C_{0}^{N}$, by Claim 2 we must have that $\delta\left(Y_{0}, s_{0}\right) \leq 2^{-N} r$. Therefore there exists a point $\left(z_{0}, \tau_{0}\right) \in \Sigma$ such that $\left\|\left(Y_{0}, s_{0}\right)-\left(z_{0}, \tau_{0}\right)\right\| \leq 2^{-N} r$.

Define $Q_{1}:=Q\left(\left(z_{0}, \tau_{0}\right), \frac{r}{2}\right)$. Then $\left(Y_{0}, s_{0}\right) \in Q_{1}$ and by Hölder continuity at the boundary we have that

$$
\begin{aligned}
C_{0}^{N} \leq u\left(Y_{0}, s_{0}\right) & \leq C\left(\frac{\delta\left(Y_{0}, s_{0}\right)}{r}\right)^{\alpha} \mathscr{f}_{Q_{1}} u \\
& \leq C 2^{-N \alpha} \sup _{Q_{1}} u .
\end{aligned}
$$

Or equivalently,

$$
\sup _{Q_{1}} u \geq C_{0}^{N} \frac{1}{C} 2^{N \alpha} \geq C_{0}^{N+1}
$$

for $N$ large enough. Hence there exists $\left(Y_{1}, s_{1}\right) \in \overline{Q_{1}}$ such that $u\left(Y_{1}, s_{1}\right) \geq C_{0}^{N+1}$. Following Claim 2, this implies that $\delta\left(Y_{1}, s_{1}\right) \leq 2^{-N-1} r$ and therefore there exists $\left(z_{1}, \tau_{1}\right) \in \Sigma$ such that $\left\|\left(Y_{1}, s_{1}\right)-\left(z_{1}, \tau_{1}\right)\right\| \leq 2^{-N-1} r$.

Define $Q_{2}:=Q\left(\left(z_{1}, \tau_{1}\right), \frac{r}{4}\right)$. Then $\left(Y_{1}, s_{1}\right) \in Q_{2}$ and by Hölder continuity at the boundary we have that

$$
\begin{aligned}
C_{0}^{N+1} \leq u\left(Y_{1}, s_{1}\right) & \leq C\left(\frac{\delta\left(Y_{1}, s_{1}\right)}{r}\right)^{\alpha} f f_{Q_{2}} u \\
& \leq C 2^{-N \alpha} \sup _{Q_{2}} u .
\end{aligned}
$$


Or equivalently,

$$
\sup _{Q_{2}} u \geq C_{0}^{N+1} \frac{1}{C} 2^{N \alpha} \geq C_{0}^{N+2}
$$

for $N$ large enough. Hence there exists $\left(Y_{2}, s_{2}\right) \in \overline{Q_{2}}$ such that $u\left(Y_{2}, s_{2}\right) \geq C_{0}^{N+1}$.

Iterating we obtain $\left\{Q_{k}\right\}$ and $\left\{\left(Y_{k}, s_{k}\right)\right\}$ such that $\delta\left(Y_{k}, s_{k}\right) \leq 2^{-N-k} r \rightarrow 0$ as $k \rightarrow \infty$ and such that

$$
\left\|\left(Y_{k}, s_{k}\right)-\left(Y_{k-1}, s_{k-1}\right)\right\| \leq \operatorname{diam}\left(Q_{k}\right)=c_{n} 2^{-k} r
$$

as $\left(Y_{k}, s_{k}\right),\left(Y_{k-1}-s_{k-1}\right) \in Q_{k}$. Therefore, $\left\{\left(Y_{k}, s_{k}\right)\right\}$ is a Cauchy sequence and hence there exists $\left(y_{*}, s_{*}\right) \in \Sigma$ such that $\left(Y_{k}, s_{k}\right) \rightarrow\left(y_{*}, s_{*}\right)$ as $k \rightarrow \infty$.

We note that for all $k,\left(Y_{k}, s_{k}\right) \in Q_{C_{n} r}\left(x_{0}, t_{0}\right)$ as

$$
\begin{aligned}
\left\|\left(Y_{0}, s_{0}\right)-\left(Y_{1}, s_{1}\right)\right\| & \leq \frac{r}{2} \\
\left\|\left(Y_{1}, s_{1}\right)-\left(Y_{2}, s_{2}\right)\right\| & \leq \frac{r}{4} \\
\vdots & \\
\left\|\left(Y_{k-1}, s_{k-1}\right)-\left(Y_{k}, s_{k}\right)\right\| & \leq \frac{r}{2^{k}}
\end{aligned}
$$

and by the triangle inequality, this implies

$$
\left\|\left(Y_{0}, s_{0}\right)-\left(Y_{k}, s_{k}\right)\right\| \leq C_{n} r
$$

where $\left(Y_{0}, s_{0}\right) \in Q_{r}\left(x_{0}, t_{0}\right)$. We also see that $\left(y_{*}, s_{*}\right) \in \Delta_{2 C_{n} r}$.

As $u\left(Y_{k}, s_{k}\right) \geq C_{0}^{N+k} \rightarrow \infty$ as $k \rightarrow \infty$, we obtain a contradiction to local boundedness.

\subsubsection{Caccioppoli's Inequality}

We follow here the argument of [LSU].

Lemma 2.0.29. Let $(Y, s) \in \Omega$ and fix $r$ with $0<r<\rho \leq \min \{\sqrt{s} / 2, \delta(Y, s)\}$. Let $Q_{r}(Y, s) \subset$ $\mathbb{R}^{n+1} \cap\{t>0\}$, and let $L$ be a divergence form parabolic operator as above. Let $u$ be a weak 
solution of $L u=0$ in $Q_{2 r}(Y, s)$, where $u \in W_{2}^{1,0}\left(Q_{2 r}(Y, s)\right)$. Then there exists $C=C(n, \lambda)$ such that

$$
\iint_{Q_{r}(Y, s)}|\nabla u|^{2} \leq \frac{C}{\ell(Q)^{2}} \iint_{Q_{2 r}(Y, s)}|u|^{2}
$$

Proof. We interpret $L u=0$ in the weak sense, i.e. for all $\varphi \in \stackrel{\circ}{W}_{2}^{1,1}\left(Q_{2 r}(Y, s)\right)$,

$$
\iint_{Q_{2 r}(Y, s)}\left(A(X, t) \nabla u(X, t) \cdot \nabla \varphi(X, t)-u(X, t) \partial_{t} \varphi(X, t)\right) d X d t=0
$$

Let $\eta \in C_{0}^{\infty}\left(Q_{3 r / 2}(Y, s)\right)$, with $\eta \equiv 1$ on $Q_{r}(Y, s), 0 \leq \eta \leq 1,\|\nabla \eta\|_{\infty} \lesssim \frac{1}{\ell(Q)}$, and $\left\|\partial_{t} \eta\right\|_{\infty} \lesssim$ $\frac{1}{\ell(Q)^{2}}$.

Consider the Steklov average of $u$, defined as follows:

$$
u_{h}(X, t):=\frac{1}{h} \int_{t}^{t+h} u(X, s) d s .
$$

By $[\mathrm{M}]$ we have that $u$ is a continuous function and applying the Fundamental Theorem of Calculus and the continuity of $u$, we obtain

$$
\partial_{t} u_{h}(X, t)=\frac{u(X, t+h)-u(X, t)}{h} \quad \text { and } \quad \lim _{h \rightarrow 0} u_{h}(X, t)=u(X, t),(X, t) \in Q_{2 r}(Y, s) .
$$

As

$$
\nabla u_{h}(X, t)=\frac{1}{h} \int_{t}^{t+h} \nabla u(X, s) d s
$$

we obtain that $u_{h} \in W_{2}^{1,1}\left(Q_{\rho}(Y, s)\right)$, and that $u_{h} \rightarrow u$ in $W_{2}^{1,0}\left(Q_{\rho}(Y, s)\right)$, for all $\rho<2 r$, provided that $h<4 r^{2}-\rho^{2}$. Using Lebesgue's Differentiation Theorem we have that

$$
\lim _{h \rightarrow 0}(A \nabla u)_{h}=A \nabla u, \quad \text { a.e. in } Q_{2 r}(Y, s),
$$

and in $L^{2}\left(Q_{\rho}(Y, s)\right)$ for all $\rho<2 r$.

Define

$$
u_{\bar{h}}(X, t):=\frac{1}{h} \int_{t-h}^{t} u(X, s) d s
$$


We make the following observations:

$$
\partial_{t} v_{\bar{h}}(X, t)=\frac{v(X, t)-v(X, t-h)}{h}=\frac{1}{h} \int_{t-h}^{t} \partial_{s} v(X, s) d s=\left(\partial_{t} v\right)_{\bar{h}}(X, t),
$$

and

$$
\nabla v_{h}(X, t)=\nabla\left(\frac{1}{h} \int_{t}^{t+h} v(X, s) d s\right)=\frac{1}{h} \int_{t}^{t+h} \nabla v(X, s) d s=(\nabla v)_{h}(X, t)
$$

Claim 1: $\iint u v_{h}=\iint u_{\bar{h}} v$ for all $u, v \in L^{2}$.

Proof of Claim 1.

$$
\begin{aligned}
\iint u(X, t) v_{h}(X, t) d X d t & =\iint u(X, t) \frac{1}{h} \int_{t}^{t+h} v(X, s) d s d X d t \\
& =\iint \frac{1}{h} \int_{s-h}^{s} u(X, t) d t v(X, s) d X d s \\
& =\iint u_{\bar{h}}(X, s) v(X, s) d X d s,
\end{aligned}
$$

where we have used Fubini's Theorem in the second equality.

Claim 2: $\iint \partial_{t} u_{h} v=-\iint u \partial_{t} v_{\bar{h}}$, for $v \in \stackrel{\circ}{W}_{2}^{1,1}\left(Q_{\rho}(Y, s)\right)$ with $\rho<2 r$ and $h$ small.

Proof of Claim 2. Applying Claim 1, (2.0.33), and using integration by parts we obtain that

$$
-\iint u \partial_{t} v_{\bar{h}}=-\iint u\left(\partial_{t} v\right)_{\bar{h}}=-\iint u_{h} \partial_{t} v=\iint \partial_{t} u_{h} v
$$

For sake of notational convenience, let $v:=u_{h} \eta^{2} \in \stackrel{\circ}{W}_{2}^{1,1}\left(Q_{3 r / 2}(Y, s)\right)$. We input $v$ into (2.0.31) to see that

$$
\iint_{Q_{2 r}(Y, s)} A \nabla u \nabla v d X d t-\iint_{Q_{2 r}(Y, s)} u \partial_{t} v d X d t=0
$$


By ellipticity we obtain that

$$
\begin{array}{r}
\iint_{Q_{r}(Y, s)}|\nabla u|^{2} d X d t \leq \iint_{Q_{2 r}(Y, s)}|\nabla u|^{2} \eta^{2} d X d t \leq \lambda^{-1} \iint_{Q_{2 r}(Y, s)} A \nabla u \cdot \nabla u \eta^{2} d X d t \\
=\lambda^{-1} \iint_{Q_{2 r}(Y, s)} A \nabla u \cdot \nabla\left(u \eta^{2}\right) d X d t-\lambda^{-1} \iint_{Q_{2 r}(Y, s)} A \nabla u \cdot \nabla \eta 2 \eta u d X d t .
\end{array}
$$

Combining (2.0.35), (2.0.32), and the fact that $u_{h} \rightarrow u$ in $W_{2}^{1,0}\left(Q_{3 r / 2}(Y, s)\right)$ we obtain that

$$
\begin{aligned}
\lambda \iint_{Q_{r}(Y, s)}|\nabla u|^{2} d X d t & \leq \lim _{h \rightarrow 0}\left(\iint_{Q_{2 r}(Y, s)}(A \nabla u)_{h} \cdot \nabla\left(u_{h} \eta^{2}\right) d X d t\right) \\
& -\iint_{Q_{2 r}(Y, s)} A \nabla u \cdot \nabla \eta 2 \eta u d X d t:=\lim _{h \rightarrow 0} I-I I .
\end{aligned}
$$

Using (2.0.33), (2.0.34), and Claim 2 we obtain that

$$
\begin{aligned}
I & =\iint_{Q_{2 r}(Y, s)}(A \nabla u)_{h} \cdot \nabla v d X d t+\iint_{Q_{2 r}(Y, s)}\left(\partial_{t} u\right)_{h} v d X d t-\iint_{Q_{2 r}(Y, s)}\left(\partial_{t} u\right)_{h} v d X d t \\
& =\iint_{Q_{2 r}(Y, s)} A \nabla u \cdot \nabla v_{\bar{h}} d X d t-\iint_{Q_{2 r}(Y, s)} u \partial_{t} v_{\bar{h}} d X d t-\iint_{Q_{2 r}(Y, s)} \partial_{t} u_{h} v d X d t \\
& =-\iint_{Q_{2 r}(Y, s)} \partial_{t} u_{h} v d X d t,
\end{aligned}
$$

where in the last equality we are applying (2.0.31) since $v_{\bar{h}} \in \stackrel{\circ}{W}_{2}^{1,1}\left(Q_{2 r}(Y, s)\right)$ for $h$ suitably small. Replacing $v$ by $u_{h} \eta^{2}$ and using integration by parts we have

$$
\begin{aligned}
\lim _{h \rightarrow 0} I & =-\lim _{h \rightarrow 0} \iint_{Q_{2 r}(Y, s)} \partial_{t} u_{h} u_{h} \eta^{2} d X d t \\
& =-\lim _{h \rightarrow 0} \frac{1}{2} \iint_{Q_{2 r}(Y, s)} \partial_{t}\left(u_{h}^{2}\right) \eta^{2} d X d t=\lim _{h \rightarrow 0} \iint_{Q_{2 r}(Y, s)} u_{h}^{2} \partial_{t} \eta \eta d X d t \\
& \leq \frac{1}{\ell(Q)^{2}} \lim _{h \rightarrow 0} \iint_{Q_{2 r}(Y, s)}\left|u_{h}\right|^{2} \eta d X d t \\
& =\frac{1}{\ell(Q)^{2}} \iint_{Q_{2 r}(Y, s)}|u|^{2} \eta d X d t
\end{aligned}
$$

Let $\epsilon>0$. Applying Cauchy's inequality with epsilon we obtain

$$
\begin{aligned}
-I I & \leq 2 \lambda^{-1} \iint_{Q_{2 r}(Y, s)}|\nabla u||\nabla \eta| \eta|u| d X d t \\
& \leq \lambda^{-1} \frac{1}{2 \epsilon} \iint_{Q_{2 r}(Y, s)}|\nabla \eta|^{2}|u|^{2} d X d t+2 \lambda^{-1} \epsilon \iint_{Q_{2 r}(Y, s)}|\nabla u|^{2} \eta^{2} d X d t
\end{aligned}
$$




$$
\leq \lambda^{-1} \frac{1}{2 \epsilon} \frac{1}{\ell(Q)^{2}} \iint_{Q_{2 r}(Y, s)}|u|^{2} d X d t+2 \lambda^{-1} \epsilon \iint_{Q_{2 r}(Y, s)}|\nabla u|^{2} \eta^{2} d X d t
$$

Therefore,

$$
\begin{aligned}
\lambda \iint_{Q_{r}(Y, s)}|\nabla u|^{2} d X d t & \leq \frac{1}{\ell(Q)^{2}} \iint_{Q_{2 r}(Y, s)}|u|^{2} \eta d X d t \\
& +\lambda^{-1} \frac{1}{2 \epsilon} \frac{1}{\ell(Q)^{2}} \iint_{Q_{2 r}(Y, s)}|u|^{2} d X d t+2 \lambda^{-1} \epsilon \iint_{Q_{2 r}(Y, s)}|\nabla u|^{2} \eta^{2} d X d t
\end{aligned}
$$

Equivalently,

$$
\left(\lambda-2 \lambda^{-1} \epsilon\right) \iint_{Q_{r}(Y, s)}|\nabla u|^{2} d X d t \leq\left(1+\lambda^{-1} \frac{1}{2 \epsilon}\right) \frac{1}{\ell(Q)^{2}} \iint_{Q_{2 r}(Y, s)}|u|^{2} d X d t
$$

Choose $\epsilon$ so that $\lambda-2 \lambda^{-1} \epsilon>0$. Then

$$
\iint_{Q_{r}(Y, s)}|\nabla u(X, t)|^{2} d X d t \lesssim \frac{1}{\ell(Q)^{2}} \iint_{Q_{2 r}(Y, s)}|u(X, t)|^{2} d X d t .
$$




\section{Chapter 3}

\section{Main Results}

\section{Recall our setting:}

We assume that $\Omega \subset \mathbb{R}^{n+1}$ is an open set whose boundary satisfies an appropriate version of a parabolic Ahlfors-David regularity condition.

We consider the heat operator

$$
L_{0}:=\partial_{t}-\mathcal{L}
$$

where $\mathcal{L}:=\nabla \cdot \nabla$ is the usual Laplacian in $\mathbb{R}^{n}$, acting in the space variables. With a caveat, to be discussed momentarily, our results may apply more generally to divergence form parabolic operators

$$
L:=\partial_{t}-\operatorname{div} A(X, t) \nabla,
$$

defined in an open set $\Omega \subset \mathbb{R}^{n+1}$ as described above, where $A$ is $n \times n$, real, $L^{\infty}$, and satisfies the uniform ellipticity condition.

Some comments are in order. As mentioned above, there is a caveat when applying our results to variable coefficient operators, namely that at present it appears to be an open problem to construct parabolic measure for such operators, in the very general class of domains that we consider here. To do so first requires that one can solve, in an appropriate sense, the Dirichlet problem with continuous data, so that parabolic measure can be constructed via Riesz representation. One can construct Perron solutions (as a supremum of 
subsolutions) but then, to apply the Riesz representation theorem, one needs linearity of the solution map (i.e., the map that sends data $f$ to the value of the solution at a given point $(X, t)$ in the domain). For the heat equation this works, since it is known that continuous functions on the parabolic boundary (or, to be more precise, on the "essential boundary"; see Definition 1.2.4) are resolutive for the heat equation (see [W1] or [W2]), and therefore the solution map is linear. On the other hand, for more general parabolic operators, linearity of the solution map would follow if one could solve the continuous Dirichlet problem, in the sense of Definition 1.2.13 above. A rather general result in this direction was obtained in $[\mathrm{CDK}]$, where the authors assume an exterior measure condition, backwards in time (see [CDK, Definition 1.3]). Otherwise, it would suffice to have a Wiener criterion to ensure continuity up to the parabolic boundary, along with enough solutions in a class to which the Wiener criterion can be applied; to our knowledge, there are versions of the parabolic Wiener test that apply to Perron solutions either for the heat equation [La], [EG], or to divergence form parabolic equations with $C^{\infty}$ or $C^{1}$-Dini coefficients [GL], [FGL], respectively; or, in the case of general divergence form parabolic operators, to some class of weak solutions (either the class $V_{2}[\mathrm{BiM}]$, or $W^{1,2}$ [GZ]). It appears to be an open problem to construct solutions of the latter sort, say for data that is Lipschitz with compact support, except in cylindrical domains [LSU], in Lip $(1,1 / 2)$ domains [BHL], and in parabolic Reifenberg flat domains [BW]. Thus, our results will apply without further qualification to the heat equation, or to operators with $C^{1}$-Dini coefficients, but at present, they will apply to general divergence form parabolic operators only if one is given a priori that the classical Dirichlet problem, with continuous data, is solvable. We observe that the capacitary conditions in [BiM, GZ] hold in our setting: they follow from the time-backwards version of 
ADR (Definition 1.2.19) that we assume; the obstacle to our applying these Wiener criteria, is the lack of solutions.

We note that for an arbitrary open set $\Omega \subset \mathbb{R}^{n+1}$, caloric measure may be constructed via the PWB method, since continuous functions on the essential boundary are resolutive; see [W1] or [W2, Chapter 8].

\subsection{A Weak Reverse Hölder Inequality for Parabolic Measure}

\subsubsection{Introduction}

It is well known that for a Lipschitz domain $\Omega$, the Dirichlet problem for a divergence form uniformly elliptic equation $L u=-\operatorname{div} A \nabla u=0$, with data in $L^{p}(\partial \Omega)$, is solvable for some $1<p<\infty$ if and only if elliptic-harmonic measure for $L$ is absolutely continuous with respect to surface measure and the Poisson kernel satisfies a reverse Hölder condition with exponent $p^{\prime}$; see $[\mathrm{Ke}]$ and the references cited there. In particular, in the case that $L$ is the Laplacian, the Poisson kernel satisfies an $L^{2}$ reverse Hölder inequality, and therefore the Dirichlet problem is solvable with data in $L^{2}(\partial \Omega)$ (see [Da]).

In this section we prove a parabolic version of a result of Bennewitz-Lewis [BL], who gave a criterion for nondoubling harmonic measure to satisfy a weak- $A_{\infty}$ condition, or equivalently, for the Poisson kernel to satisfy a weak reverse Hölder condition; see Definition 1.2.37 above. To put this work in context, we recall that David-Jerison [DJ] and Semmes $[\mathrm{S}]$ proved that harmonic measure $\omega$ on the boundary of an NTA domain with Ahlfors-David regular boundary is $A_{\infty}$ with respect to surface measure. The idea of the approach in [DJ] is first to prove a geometric result, whereby domains satisfying a certain two sided interior and exterior thickness condition (that is, the two sided "Corkscrew" condition), and having ADR boundaries, could be approximated in a "Big Pieces" sense by 
Lipschitz sub-domains. As a consequence, by the maximum principle combined with the fundamental result of [Da], one obtains a certain local ampleness property of the harmonic measure (see (3.1.2) below for the parabolic version), which may then, in the presence of the Harnack chain condition, be self-improved to give the $A_{\infty}$ property.

In $[\mathrm{BL}]$, the authors show that this self-improvement procedure, i.e., the passage from local ampleness of harmonic measure to quantitative absolute continuity, can still be executed, even in the absence of the Harnack chain condition, and as a consequence are able to extend the result of [DJ] and [S], in an appropriate way, to much more general domains. They are able to conclude only that harmonic measure is weak- $A_{\infty}$ with respect to surface measure, but on the other hand, this conclusion is best possible: their results apply to domains in which harmonic measure need not be doubling (in particular, to the case that the domain satisfies a uniform interior big pieces of Lipschitz graph condition and an interior corkscrew condition, but no connectivity property, such as the Harnack chain condition).

The goal of the present section is to extend the results of $[\mathrm{BL}]$ to the parabolic setting. As regards geometric hypotheses, we assume only that $\Omega \subset \mathbb{R}^{n+1}$ is an open set whose boundary satisfies an appropriate version of a parabolic Ahlfors-David regularity condition. In particular, we impose no connectivity hypothesis, such as a parabolic Harnack chain condition. We may then consider the initial-Dirichlet problem in subdomains of the form $\Omega^{T}=\Omega \cap\{t>T\}$, for appropriate fixed times $T$. We shall return to the latter point below.

\subsubsection{Main Result}

For a sufficiently large (and eventually fixed) constant $K_{1}$, given $(X, t) \in \Omega$, set

$$
Q_{X, t}:=Q\left((X, t), K_{1} \delta(X, t)\right), \quad \Delta_{X, t}:=Q_{X, t} \cap \Sigma,
$$


where in general the parabolic cube $Q((X, t), r)$ is defined as in (1.2.3) above. Our results will hold for $K_{1}$ in (3.1.1) large enough, depending only on the constants in Lemma 2.0.5.

For $(X, t) \in \Omega$, and $\Delta_{X, t}$ defined as in (3.1.1), we shall say that caloric (or parabolic) measure $\omega^{X, t}$ is locally ample on $\Delta_{X, t}$, or more precisely, $(\theta, \beta)$-locally ample, if there exists constants $\theta, \beta \in(0,1)$ such that

$$
\sigma(E) \geq(1-\theta) \sigma\left(\Delta_{X, t}\right) \quad \Longrightarrow \quad \omega^{X, t}(E)=\omega_{L}^{X, t}(E) \geq \beta
$$

where $E \subset \Delta_{X, t}$ is a Borel set. We observe that if (3.1.2) holds for some $K_{1}=K \geq 2$, then it also holds with $K_{1}=K^{\prime}>K$, for some $\theta^{\prime}=\theta^{\prime}\left(\theta, K, K^{\prime}, n, A D R\right)$. Thus, we may always fix a larger value of $K_{1}$, at our convenience.

Set $T_{\text {min }}:=\inf \{T: \Omega \cap\{t \equiv T\} \neq \emptyset\}$ (note that we may have $T_{\text {min }}=-\infty$ ).

Theorem 3.1.3. Let $\Omega \subset \mathbb{R}^{n+1}$ be an open set whose quasi-lateral boundary $\Sigma$ is globally ADR. Let $\left(x_{0}, t_{0}\right) \in \Sigma$, and let $0<r<\sqrt{t_{0}-T_{\min }} /(8 \sqrt{n})$. Assume that $\Sigma$ is time-backwards $A D R$ on $\Delta_{2 r}=\Sigma \cap Q_{2 r}\left(x_{0}, t_{0}\right)$, and suppose that there are constants $\theta, \beta \in(0,1)$, and $a$ value of $K_{1} \geq 2$ in (3.1.1), such that caloric measure $\omega^{X, t}$ satisfies the $(\theta, \beta)$-local ampleness condition (3.1.2) on $\Delta_{X, t}$ for each $(X, t) \in \Omega \cap Q_{2 r}\left(x_{0}, t_{0}\right)$.

Then there exist constants $C \geq 1, \gamma>0$, such that if $\left(Y_{0}, s_{0}\right) \in \Omega \backslash Q_{4 r}\left(x_{0}, t_{0}\right)$, then $\omega^{Y_{0}, s_{0}} \ll \sigma$ on $\Sigma \cap Q_{r}\left(x_{0}, t_{0}\right)$, with $d \omega^{Y_{0}, s_{0}} / d \sigma=h$ satisfying

$$
\begin{aligned}
&\left(\rho^{-n-1} \iint_{\Delta_{\rho}(y, s)} h^{1+\gamma} d \sigma\right)^{1 /(1+\gamma)} \leq C \rho^{-n-1} \iint_{\Delta_{2 \rho}(y, s)} h d \sigma \\
&=C \rho^{-n-1} \omega^{Y_{0}, s_{0}}\left(\Delta_{2 \rho}(y, s)\right),
\end{aligned}
$$

whenever $(y, s) \in \Sigma$ and $Q_{2 \rho}(y, s) \subset Q_{r}\left(x_{0}, t_{0}\right)$, where $\Delta_{\rho}(y, s)=Q_{\rho}(y, s) \cap \Sigma$, and $\Delta_{2 \rho}(y, s)=$ $Q_{2 \rho}(y, s) \cap \Sigma$. 
Moreover, the same result applies to the parabolic measure associated to a uniformly parabolic divergence form operator $L$, provided that the continuous Dirichlet problem is solvable for L in $\Omega$ (see Definition 1.2.13).

To clarify matters, we remark that by the ADR hypothesis on $\Sigma$ (see Definition 1.2.17), we have that $\rho^{n+1} \approx \sigma\left(\Delta_{\rho}(y, s)\right) \approx \sigma\left(\Delta_{2 \rho}(y, s)\right)$. The time-backwards ADR condition is an enhanced version of ADR, which entails some thickness of $\Sigma$ backwards in time; see Definition 1.2.19, and Remarks 1.2.21, 1.2.23, and 1.2.25.

Similar results in the parabolic setting have previously been established under the more restrictive assumptions that 1) $L$ is the heat operator and the lateral boundary of the domain is given locally as the graph of a function $\psi(x, t)$ which is Lipschitz in the space variable, and has a 1/2-order time derivative in parabolic BMO $[\mathrm{LM}]^{1}$, 2) $\Omega=\left\{\left(x_{0}, x, t\right) \in\right.$ $\left.(0, \infty) \times \mathbb{R}^{n-1} \times \mathbb{R}\right\}$ is a half-space and the coefficients of $L$ satisfy a certain Carleson measure regularity property [HL], and 3) $L$ is the heat operator and either $\Omega$ is a parabolic Reifenberg flat domain [HLN], or $\Omega$ is a parabolic chord-arc domain [NS]; in each of these settings, $\Omega$ enjoys a parabolic version of the Harnack Chain condition, which entails a rather strong quantitative version of connectivity. As mentioned above, the elliptic analogue of our result was proved in $[\mathrm{BL}]$, without any connectivity hypothesis. The new contribution of the present result is to dispense with all connectivity assumptions, both qualitative and quantitative, in the parabolic setting. The elliptic version obtained in [BL] has proved to be useful in various applications, see, e.g., [HLe] and [HM]. We shall discuss two applications of our work in the sequel.

\footnotetext{
${ }^{1}$ For domains whose lateral boundary is given locally as a graph, the $1 / 2$ order derivative in BMO condition of $[\mathrm{LM}]$ is in the nature of best possible: there is a counterexample of Kaufmann and Wu [KW], with $\psi \in$ $\mathrm{Lip}_{1 / 2}$ in the time variable.
} 


\subsubsection{Lemmas \& Proof}

In this section, we state and prove two lemmas, which we then use to prove Theorem 3.1.3.

Lemma 3.1.5. Let $\left(x_{0}, t_{0}\right) \in \Sigma$, and let $0<r<\sqrt{t_{0}-T_{\min }} /(8 \sqrt{n})$. Suppose that $\Sigma$ is time-backwards $A D R$ on $\Delta_{2 r}:=Q_{2 r}\left(x_{0}, t_{0}\right) \cap \Sigma$. Suppose further that there exist constants $\theta, \beta \in(0,1)$ such that $\omega^{X, t}$ satisfies the $(\theta, \beta)$-local ampleness condition (3.1.2) on $\Delta_{X, t}$ for $\operatorname{each}(X, t) \in \Omega \cap Q_{2 r}\left(x_{0}, t_{0}\right)$.

Then given $\epsilon>0$, there exists $\eta=\eta(\epsilon, n), 0<\eta<1$ and $C_{\epsilon}=C(\epsilon, n) \geq 1$ such that for any Borel set $F \subset \Delta_{2 r}$, with $\sigma(F) \geq(1-\eta) \sigma\left(\Delta_{2 r}\right)$, we have

$$
\omega^{Y, s}\left(\Delta_{r}\left(x_{0}, t_{0}\right)\right) \leq \epsilon \omega^{Y, s}\left(\Delta_{2 r}\left(x_{0}, t_{0}\right)\right)+C_{\epsilon} \omega^{Y, s}(F),
$$

whenever $(Y, s) \in \Omega \backslash Q_{4 r}\left(x_{0}, t_{0}\right)$.

Remark 3.1.7. $M_{1}, a$ are the constants from Lemma 2.0.5.

Proof of Lemma 3.1.5. The proof is an adaption of the argument in [BL] to the parabolic setting. The principal new difficulty is the time lag inherent in parabolic problems.

Fix $\epsilon>0, r>0$, and $\left(x_{0}, t_{0}\right) \in \Sigma$, with $0<r<\sqrt{t_{0}-T_{\min }} /(8 \sqrt{n})$, and suppose that $\Sigma$ is TBADR on $\Delta_{2 r}=\Delta_{2 r}\left(x_{0}, t_{0}\right)=Q_{2 r}\left(x_{0}, t_{0}\right) \cap \Sigma$. Observe that if (3.1.6) is true for some $\epsilon>0$, then it is true for all $\tilde{\epsilon}>\epsilon$. Thus, we may suppose that $\epsilon \leq \epsilon_{0}$ for some sufficiently small but fixed $\epsilon_{0}>0$.

Recall that $\delta(\mathbf{Y}):=\operatorname{dist}\left(\mathbf{Y}, \partial_{e} \Omega\right)$, where the distance is of course the parabolic distance. Replacing $Q_{r}$ by $Q_{2 r}$ in Remark 1.2.24, we have

$$
r<\sqrt{t_{0}-T_{\min }} /(8 \sqrt{n}) \Longrightarrow \delta(\mathbf{Y})=\operatorname{dist}(\mathbf{Y}, \Sigma), \quad \forall \mathbf{Y} \in \Omega \cap Q_{2 r}\left(x_{0}, t_{0}\right)
$$

Thus, by hypothesis, we shall be working in a regime where $\delta(\mathbf{Y})=\operatorname{dist}(\mathbf{Y}, \Sigma)$. Moreover, 
we note that in this regime, i.e., for $(Y, s) \in \Omega \cap Q_{2 r}\left(x_{0}, t_{0}\right)$, we have

$$
\operatorname{dist}((Y, s), \Sigma)=\delta(Y, s) \approx_{a} \operatorname{dist}((Y, s), \partial \Omega), \quad \text { if } s<t_{0}-(a r)^{2}
$$

since $\left(x_{0}, t_{0}\right) \in \Sigma$ implies that $t_{0} \leq T_{\max }$. Let us further note that $s<T_{\max }$, for $(Y, s) \in$ $\Omega \cap Q_{2 r}\left(x_{0}, t_{0}\right)$, hence by Remark 1.2.23,

$$
Q_{\rho}^{-}(Y, s) \cap \partial_{s} \Omega=\emptyset, \quad 0<\rho<r / 2, \quad(Y, s) \in \Omega \cap Q_{3 r / 2}\left(x_{0}, t_{0}\right) .
$$

We shall use these facts repeatedly in the sequel, often implicitly.

Let $M$ be a large positive constant to be chosen later. Since it suffices to work with suitably small $\epsilon$, we may suppose that $\epsilon \leq M^{-2}$. Let $j$ be the greatest integer $\leq M / \epsilon$. Let

$$
\begin{aligned}
r_{k}^{*} & =\left(\frac{5}{4}+\frac{k}{4 j}\right) r, \\
r_{k}^{\prime} & =\left(\frac{5}{4}+\frac{k+1}{4 j}\right) r \\
\widehat{r}_{k} & =\left(\frac{5}{4}+\frac{k+1 / 2}{4 j}\right) r
\end{aligned}
$$

Then define

$$
U_{k}:=Q_{r_{k}^{\prime}}\left(x_{0}, t_{0}\right) \backslash Q_{r_{k}^{*}}\left(x_{0}, t_{0}\right), \quad \text { and } \quad S_{k}:=\partial Q_{\widehat{r}_{k}}\left(x_{0}, t_{0}\right) \cap \Omega
$$

for $1 \leq k \leq j-1$. Note that $S_{k} \subset U_{k}$ and $U_{k} \subset Q_{\frac{3}{2} r}\left(x_{0}, t_{0}\right)$ for each $k$.

Let $\epsilon^{\prime}:=\frac{a}{M M_{1}} \epsilon$, and let $F \subset \Delta_{2 r}$, with

$$
\sigma(F) \geq(1-\eta) \sigma\left(\Delta_{2 r}\right)
$$

where $\eta=\eta(\epsilon)>0$ will be chosen momentarily. We begin with two preliminary observations.

Remark 3.1.12. First, assume that $\left(Y_{1}, s_{1}\right) \in Q_{\frac{7}{4} r}\left(x_{0}, t_{0}\right)$ with

$$
\frac{\epsilon^{\prime}}{400} r \leq \delta\left(Y_{1}, s_{1}\right) \leq \frac{\epsilon^{\prime}}{100} r
$$


Recalling (3.1.1) and fixing $K_{1}:=20 a^{-1} M_{1}$, we set $Q_{Y_{1}, s_{1}}:=Q\left(\left(Y_{1}, s_{1}\right), 20 \frac{M_{1}}{a} \delta\left(Y_{1}, s_{1}\right)\right)$, so that $\Delta_{Y_{1}, s_{1}}=\Sigma \cap Q_{Y_{1}, s_{1}}$, and note that $Q_{Y_{1}, s_{1}} \subset Q_{\frac{15}{8} r}\left(x_{0}, t_{0}\right)$. Then for $\eta(\epsilon)$ small enough,

$$
\sigma\left(F \cap Q_{Y_{1}, s_{1}}\right) \geq(1-\theta) \sigma\left(\Delta_{Y_{1}, s_{1}}\right)
$$

Hence, by the local-ampleness assumption, setting $C=1 / \beta$, we have

$$
C \omega^{Y_{1}, s_{1}}(F) \geq 1, \quad\left(Y_{1}, s_{1}\right) \in Q_{\frac{7}{4} r}\left(x_{0}, t_{0}\right) \cap\left\{(Y, s): \frac{\epsilon^{\prime}}{400} r \leq \delta(Y, s) \leq \frac{\epsilon^{\prime}}{100} r\right\}
$$

Note that our assumption in Theorem 3.1.3 is that $(\theta, \beta)$-local ampleness holds for some value of $K_{1} \geq 2$. If this $K_{1}$ exceeds the value defined above, then we simply take $M_{1}$ larger. On the other hand, as noted previously, if $(\theta, \beta)$-local ampleness holds for a smaller value of $K_{1} \geq 2$ than that defined above, then it also holds for larger $K_{1}$ (for a possibly different value of $\theta$ ). In any case, we are at liberty to fix $K_{1}$ as above.

Remark 3.1.14. Next, suppose that $(Y, s) \in S_{k}$, with $\delta(Y, s)<\epsilon^{\prime} r / 200$. Then

$$
Q_{Y, s}:=Q\left((Y, s), 20 \frac{M_{1}}{a} \delta(Y, s)\right) \subset U_{k}
$$

By Lemma 2.0.5,

$$
\omega^{Y, s}\left(Q_{Y, s} \cap \Sigma\right) \gtrsim 1
$$

and therefore,

$$
\omega^{Y, s}\left(U_{k}\right) \gtrsim 1
$$

for $(Y, s) \in S_{k} \cap\left\{(Y, s): \delta(Y, s)<\epsilon^{\prime} r / 200\right\}$.

We now consider several cases. Recall that we have oriented our coordinate axes so that time runs from left to right. Given a cube $Q=Q_{\rho}\left(x_{0}, t_{0}\right)$, we shall use the terminology "back face of the boundary of $Q$ " to denote the left-most face of $\partial Q$, i.e., the face with $t=t_{0}-\rho^{2}$ 
Case 1. There is a point $\left(Y_{0}, s_{0}\right)$ on the back face of $S_{k}$ such that $\delta\left(Y_{0}, s_{0}\right)=\frac{\epsilon^{\prime}}{200} r$. Then

$$
\frac{\epsilon^{\prime}}{400} r \leq \delta\left(Y_{0}, s_{0}-c_{1} r^{2}\right) \leq \frac{\epsilon^{\prime}}{100} r
$$

where $c_{1}:=\left(\frac{\epsilon^{\prime}}{200}\right)^{4}$, and $\left(Y_{0}, s_{0}-c_{1} r^{2}\right) \in Q_{\frac{7}{4}} r\left(x_{0}, t_{0}\right)$. Hence, by (3.1.13),

$$
C \omega^{Y_{0}, s_{0}-c_{1} r^{2}}(F) \geq 1
$$

Claim 1. In the scenario of Case 1 , given $\epsilon>0$, there exists a uniform constant $C_{\epsilon}$ such that

$$
C_{\epsilon} \omega^{Y, s}(F) \geq 1, \quad \forall(Y, s) \in S_{k} \cap\left\{(Y, s): \delta(Y, s) \geq \frac{\epsilon^{\prime}}{200} r\right\}
$$

Proof of Claim 1. Let $(Y, s) \in S_{k}$ with $\delta(Y, s) \geq \frac{\epsilon^{\prime}}{200} r$.

Case 1a. $\delta(Y, s) \leq \frac{\epsilon^{\prime}}{100} r$. Then we are done by (3.1.13) (in this case with no dependence on $\epsilon)$.

Case 1b. $\delta(Y, s)>\frac{\epsilon^{\prime}}{100} r$. Note that $s \geq s_{0}$, since $\left(Y_{0}, s_{0}\right)$ lies on the back face of $S_{k}$. Consider the parabola, call it $C$, with vertex $\left(Y_{0}, s_{0}-c_{1} r^{2}\right)$, which opens to the right, contains the point $(Y, s)$, and has aperture $1 / \alpha$, with

$$
\alpha=\frac{s-\left(s_{0}-c_{1} r^{2}\right)}{\left|Y_{0}-Y\right|^{2}} \geq \frac{c_{1} r^{2}}{\left|Y-Y_{0}\right|^{2}} \gtrsim c_{1} .
$$

Of course, if $Y=Y_{0}$, then the parabola degenerates, and $C$ is just a horizontal line, parallel to the $t$-axis. In any case, we start at the point $(Y, s)$ and move backward on $C$, stopping the first time that we reach a point $\left(Z_{0}, \tau_{0}\right)$ with $\delta\left(Z_{0}, \tau_{0}\right)=\epsilon^{\prime} r / 100$. We eventually find such a point, since $\delta(Y, s)>\epsilon^{\prime} r / 100 \geq \delta\left(Y_{0}, s_{0}-c_{1} r^{2}\right)$, by the scenario of Case $1 \mathrm{~b}$ and (3.1.16). Note that by (3.1.10), all the points on $C$ between (and including) $(Y, s)$ and $\left(Z_{0}, \tau_{0}\right)$ lie in $\Omega$, and thus in particular (3.1.13) holds with $\left(Y_{1}, s_{1}\right)=\left(Z_{0}, \tau_{0}\right)$. Moreover, since $\left\|(Y, s)-\left(Z_{0}, \tau_{0}\right)\right\| \lesssim r$, and $\delta(Z, \tau) \gtrsim_{\epsilon} r$ for every $(Z, \tau) \in C$ between $(Y, s)$ and $\left(Z_{0}, \tau_{0}\right)$, 
again using (3.1.10), we can construct a Harnack path joining $\left(Z_{0}, \tau_{0}\right)$ to $(Y, s)$ along $C$, to obtain (3.1.17) by Harnack's inequality [M, Theorem 1]. This proves Claim 1.

Case 2. For every point $(Y, s)$ on the back face of $S_{k}$, we have $\delta(Y, s)>\epsilon^{\prime} r / 200$.

In this case, we slide the back face of $S_{k}$ forward in time until we reach, for the first time, a point $\left(Y_{0}, s_{0}\right)$ with $\delta\left(Y_{0}, s_{0}\right)=\epsilon^{\prime} r / 200$. Note that necessarily,

$$
s_{0} \leq \inf \left\{t:(x, t) \in \Delta_{r}\left(x_{0}, t_{0}\right) \text { for some } x\right\}-\left(\epsilon^{\prime} r / 200\right)^{2}
$$

(otherwise, we would have stopped sooner when sliding the back face forward). In particular, by Remark 1.2.25,

$$
s_{0}<t_{0}-(a r)^{2} .
$$

If we denote the boundary of the resulting rectangle by $S_{k}^{\prime}$, then by construction $\left(Y_{0}, s_{0}\right)$ is on the back face of $S_{k}^{\prime}$. Since (3.1.9) holds for the point $\left(Y_{0}, s_{0}\right)$, we may then repeat the argument in Case 1, but with $S_{k}^{\prime}$ in place of $S_{k}$. Consequently, we have the following.

Remark 3.1.19. Estimate (3.1.17) holds, provided either that

$$
(Y, s) \in S_{k} \cap\left\{(Y, s): \delta(Y, s) \geq \frac{\epsilon^{\prime}}{200} r\right\}, \quad \text { or } \quad(Y, s) \in S_{k}^{\prime} \cap\left\{(Y, s): \delta(Y, s) \geq \frac{\epsilon^{\prime}}{200} r\right\},
$$

in the scenarios of Case 1 and Case 2, respectively. Moreover, in Case 2, $t=s_{0}$ on the back face of $S_{k}^{\prime}$, and by (3.1.18), $s_{0}<t$ for any $(x, t) \in \Delta_{r}\left(x_{0}, t_{0}\right)=Q_{r}\left(x_{0}, t_{0}\right) \cap \Sigma$.

Case 3. For every $(Y, s)$ on the back face of $S_{k}$, we have $\delta(Y, s)<\epsilon^{\prime} r / 200$.

In turn, there are two sub-cases.

Case 3a: For every point $(Y, s)$ on $S_{k}$, we have $\delta(Y, s)<\epsilon^{\prime} r / 200$. In this case, the scenario of Remark 3.1.14 applies to every point $(Y, s) \in S_{k}$, and therefore (3.1.15) holds for all points on $S_{k}$. 
Case 3b. There exists a point $\left(Y^{*}, s^{*}\right)$ on $S_{k}$, with $\delta\left(Y^{*}, s^{*}\right)=\frac{\epsilon^{\prime}}{200} r$.

Recall that $S_{k}=\partial Q_{\widehat{r}_{k}}\left(x_{0}, t_{0}\right)$, where $\widehat{r}_{k}=(5 / 4+(k+1 / 2) /(4 j)) r$. Consider the part of $Q_{\widehat{r}_{k}}\left(x_{0}, t_{0}\right)$ where $t_{0}-\widehat{r}_{k}^{2}<s<t_{0}-r^{2}$ and call this region $\widehat{I}_{k}$. Note that $\left|\widehat{I}_{k}\right| \approx r^{n+2}$. Cover $\widehat{I}_{k}$ by a union of pairwise non-overlapping half-closed sub-cubes $\left\{Q^{i}\right\}_{i}$, such that $\epsilon^{\prime} r \leq l\left(Q^{i}\right) \leq 2 \epsilon^{\prime} r$

Claim 2: For $\epsilon$ small enough, at least one of the sub-cubes $Q^{i}$ misses $\Sigma$ (and thus also $\partial \Omega$ by (3.1.8) and the fact that $\left.t_{0} \leq T_{\max }\right)$.

Assume the claim momentarily. Then there exists a point $(Z, \tau) \in Q^{i}$ such that $\delta(Z, \tau)>>$ $\epsilon^{\prime} r / 200$. In the present scenario, $\delta(Y, s)<\epsilon^{\prime} r / 200$ for every $(Y, s)$ on the back face of $S_{k}$, thus, there exist $\tilde{s}$ with $s=t_{0}-\widehat{r}_{k}^{2}<\tilde{s}<\tau$, and $\delta(Z, \tilde{s})=\epsilon^{\prime} r / 200$. If we shrink $S_{k}$ by sliding the back face forward, increasing the time coordinate of the back face to $\tilde{s}$, and denote the boundary of the resulting rectangle by $S_{k}^{\prime}$, then by construction, $(Z, \tilde{s})$ lies on the back face of $S_{k}^{\prime}$. We can therefore follow the argument given in Cases 1 and 2, with $(Z, \tilde{s})$ playing the role of $\left(Y_{0}, s_{0}\right)$, to find that Remark 3.1 .19 continues to apply in Case $3 \mathrm{~b}$ as well. We note in particular that by construction $t=\tilde{s}$ on the back face of $S_{k}^{\prime}$, and $\tilde{s}<t_{0}-r^{2}<t$, for all $(x, t) \in Q_{r}\left(x_{0}, t_{0}\right) \cap \Sigma$.

Proof of Claim 2. Suppose not. Then every $Q^{i}$ meets $\Sigma$. Hence there exists another parabolic cube $\widetilde{Q}^{i}$ such that $\widetilde{Q}^{i} \supset Q^{i}, l\left(\widetilde{Q}^{i}\right)=4 l\left(Q^{i}\right)$ and the center of $\widetilde{Q}^{i}$ is on $\Sigma$. By a rudimentary covering lemma argument, there exists a pairwise disjoint subcollection $\left\{\widetilde{Q}^{i_{j}}\right\}_{j}$, with cardinality $\#\left\{Q^{i_{j}}\right\}_{j} \approx \#\left\{Q^{i}\right\}_{i}$, such that

$$
\bigcup_{j} 5 \widetilde{Q}^{i_{j}} \supset \bigcup_{i} Q^{i}=\widehat{I}_{k}
$$

Let $\widetilde{I}_{k}$ be a fattened version of $\widehat{I}_{k}$, of comparable dimensions, such that $\bigcup_{j} \widetilde{Q}^{i_{j}} \subset \widetilde{I}_{k}$. Then 
using disjointness and upper ADR, we obtain

$$
\sum_{j} \sigma\left(\widetilde{Q}^{i_{j}} \cap \Sigma\right) \leq \sigma\left(\widetilde{I}_{k} \cap \Sigma\right) \lesssim M_{0} r^{n+1} .
$$

Using lower ADR, we obtain

$$
\sum_{j} \sigma\left(\widetilde{Q}^{i_{j}} \cap \Sigma\right) \gtrsim \#\left\{\widetilde{Q}^{i_{j}}\right\}\left(\epsilon^{\prime} r\right)^{n+1}
$$

where as above, $\#\left\{\widetilde{Q}^{i_{j}}\right\}=$ cardinality of $\left\{Q^{i_{j}}\right\}$. However,

$$
\#\left\{\widetilde{Q}^{i_{j}}\right\} \approx \#\left\{Q^{i}\right\} \approx \frac{r^{n+2}}{\left(\epsilon^{\prime} r\right)^{n+2}} \approx\left(\epsilon^{\prime}\right)^{-n-2}
$$

Therefore (3.1.21) becomes

$$
\sum_{j} \sigma\left(\widetilde{Q}^{i_{j}} \cap \Sigma\right) \gtrsim\left(\epsilon^{\prime}\right)^{-n-2}\left(\epsilon^{\prime} r\right)^{n+1} \approx \frac{r^{n+1}}{\epsilon^{\prime}}>>M_{0} r^{n+1},
$$

for $\epsilon^{\prime} \approx \epsilon$ small enough, contradicting (3.1.20).

Combining Remarks 3.1.14 and 3.1.19, and our observation that the latter remark continues to hold in Case $3 \mathrm{~b}$, we see that for $(Y, s) \in S_{k}$ (in Cases 1 and $3 \mathrm{a}$ ), or $(Y, s) \in S_{k}^{\prime}$ (in Cases 2 and $3 b)$,

$$
1 \leq C \omega^{Y, s}\left(U_{k}\right)+C_{\epsilon} \omega^{Y, s}(F)
$$

Moreover, letting $t_{k}$ denote the value of $t$ on the back face of $S_{k}$ or $S_{k}^{\prime}$, as appropriate, we see that in every case, $t_{k}<t$ for every $(x, t) \in \Delta_{r}\left(x_{0}, t_{0}\right):=Q_{r}\left(x_{0}, t_{0}\right) \cap \Sigma$. Consequently, by the weak maximum principle,

$$
\omega^{Y, s}\left(\Delta_{r}\left(x_{0}, t_{0}\right)\right) \leq C \omega^{Y, s}\left(U_{k}\right)+C_{\epsilon} \omega^{Y, s}(F),
$$

for every $(Y, s) \in \Omega_{k}:=\left(\Omega \backslash R_{k}\right) \cap\left\{s>t_{k}\right\}$, where $R_{k}$ is the closed cube, or rectangle, whose boundary is given by $S_{k}$ or $S_{k}^{\prime}$. In addition, in every case, $t_{k}<t_{0}-(a r)^{2}$, and $R_{k} \subset Q_{4 r}\left(x_{0}, t_{0}\right)$, so in particular, in (3.1.23), we may take

$$
(Y, s) \in\left(\Omega \backslash Q_{4 r}\left(x_{0}, t_{0}\right)\right) \cap\left\{s>t_{0}-(a r)^{2}\right\} .
$$


For $(Y, s)$ in the latter set, we sum (3.1.23) in $k$ to obtain

$$
\epsilon^{-1} \omega^{Y, s}\left(\Delta_{r}\left(x_{0}, t_{0}\right)\right) \leq C \omega^{Y, s}\left(\Delta_{2 r}\left(x_{0}, t_{0}\right)\right)+C_{\epsilon} \omega^{Y, s}(F)
$$

since the sets $U_{k}$ are disjoint and are all contained in $Q_{2 r}\left(x_{0}, t_{0}\right)$, and the cardinality of the index set $\{k\}$ is of the order $1 / \epsilon$. We now multiply by $\epsilon$ to reach the conclusion of the lemma in the special case that $s>t_{0}-(a r)^{2}$.

Let us now remove the latter restriction. Recall that the set $E(T)$ is defined in (2.0.4). Observe that $t>t_{0}-r^{2}$ for every $(x, t) \in \Delta_{r}\left(x_{0}, t_{0}\right)$, so that if $s \leq t_{0}-r^{2}$, then $\omega^{Y, s}\left(\Delta_{r}\left(x_{0}, t_{0}\right)\right)=$ 0 , and there is nothing to prove. It therefore remains to treat the case $t_{0}-r^{2}<s \leq t_{0}-(a r)^{2}$. In this case, by an elementary covering argument, we may cover the set $\Delta_{r}\left(x_{0}, t_{0}\right) \cap E(s)$ by a collection of surface cubes $\left\{\Delta_{i}\right\}_{i=1}^{N}, \Delta_{i}=Q_{i} \cap \Sigma$, where $\Delta_{i}=\Delta_{c r}\left(x_{i}, t_{i}\right)$, with $\left(x_{i}, t_{i}\right) \in \Sigma$, $t_{i} \leq s$, and where $c$ is a universal constant chosen small enough that $2 Q_{i} \subset Q_{2 r}\left(x_{0}, t_{0}\right)$; moreover, this can be done in such a way that the cardinality $N$ of the collection is bounded by a universal constant (depending on dimension). Thus, choosing $\eta^{\prime}>0$ small enough, depending on $c$ and our previous choice of $\eta$, we have that for $F \subset \Delta_{2 r}\left(x_{0}, t_{0}\right)$,

$$
\sigma(F) \geq\left(1-\eta^{\prime}\right) \sigma\left(\Delta_{2 r}\left(x_{0}, t_{0}\right)\right) \Longrightarrow \sigma\left(F \cap 2 \Delta_{i}\right) \geq(1-\eta) \sigma\left(2 \Delta_{i}\right)
$$

Since $s \geq t_{i}$ (hence in particular $s>t_{i}-(a c r)^{2}$ ), we may therefore apply the previously treated special case to each $\Delta_{i}$, to deduce that

$$
\begin{aligned}
\omega^{Y, s}\left(\Delta_{r}\left(x_{0}, t_{0}\right)\right)= & \omega^{Y, s}\left(\Delta_{r}\left(x_{0}, t_{0}\right) \cap E(s)\right) \\
\leq \sum_{i=1}^{N} \omega^{Y, s}\left(\Delta_{i}\right) \leq \epsilon \sum_{i=1}^{N} \omega^{Y, s}\left(2 \Delta_{i}\right)+C_{\epsilon} \sum_{i=1}^{N} \omega^{Y, s}\left(F \cap 2 \Delta_{i}\right) & \leq N \epsilon \omega^{Y, s}\left(\Delta_{2 r}\left(x_{0}, t_{0}\right)\right)+N C_{\epsilon} \omega^{Y, s}(F) .
\end{aligned}
$$


The following lemma and its proof are an adaption of the argument in [BL] to the parabolic setting.

Lemma 3.1.24. Let $\Sigma$ be a closed $A D R$ set with constant $M_{0}$. Let $\mu$ be a positive Borel measure on $\mathbb{R}^{n+1}$ with support contained in $\Sigma$, and $\mu(\Sigma) \leq 1$. Suppose for some $(x, t) \in \Sigma$, $r>0$, there exists $\epsilon_{1}, \zeta>0, C_{1} \geq 1$ such that

$$
\mu\left(Q_{\rho}(y, s)\right) \leq \epsilon_{1} \mu\left(Q_{2 \rho}(y, s)\right)+C_{1} \mu(P)
$$

whenever $P \subset \Sigma \cap Q_{2 \rho}(y, s)$ is a Borel set with

$$
\frac{\sigma(P)}{\sigma\left(Q_{2 \rho}(y, s) \cap \Sigma\right)} \geq 1-\zeta
$$

and $(y, s) \in \Sigma$ with $Q_{2 \rho}(y, s) \subset Q_{r}(x, t)$.

$$
\text { If } \epsilon_{1}=\epsilon_{1}\left(n, M_{0}\right)>0 \text { is small enough, then there exists } C=C\left(n, M_{0}, c_{1}, \zeta\right), 1 \leq C<
$$
$\infty, \gamma=\gamma\left(n, M_{0}, c_{1}, \zeta\right)>0$ and a Borel measurable function $g$ such that $d \mu / d \sigma=g$ on $\Sigma \cap Q_{r}(x, t)$ while

$$
\begin{array}{rl}
\left(\rho^{-n-1} \iint_{Q_{\rho}(y, s) \cap \Sigma} g^{1+\gamma} d \sigma\right)^{1 /(1+\gamma)} \leq C \rho^{-n-1} \iint_{Q_{2 \rho}(y, s) \cap \Sigma} g & d \sigma \\
=C \rho^{-n-1} \mu\left(Q_{2 \rho}(y, s)\right) .
\end{array}
$$

Proof of Lemma 3.1.24. We first make the following claim.

Claim. $\mu<<\sigma$ on $\Delta_{r}(x, t)$.

Proof of Claim. We will first show that for any fixed $K>2^{n+1}$ and $\mu$ a.e. $(y, s) \in \operatorname{supp} \mu \subset \Sigma$

$$
\liminf _{\rho \rightarrow 0} \frac{\mu\left(Q_{2 \rho}(y, s)\right)}{\mu\left(Q_{\rho}(y, s)\right)}<K
$$

If (3.1.28) were false, then there exists $E$, a Borel measurable set, with $\mu(E)>0$ on which

$$
\liminf _{\rho \rightarrow 0} \frac{\mu\left(Q_{2 \rho}(y, s)\right)}{\mu\left(Q_{\rho}(y, s)\right)} \geq K, \quad \forall(y, s) \in E
$$


I.e., for $\varepsilon<K-2^{n+1}$, and for every $(y, s) \in E$, there exists $\rho_{0}=\rho_{0}(y, s)$ such that for all $\rho \leq \rho_{0}$,

$$
\frac{\mu\left(Q_{2 \rho}(y, s)\right)}{\mu\left(Q_{\rho}(y, s)\right)}>K-\varepsilon:=\widetilde{K}>2^{n+1} .
$$

Then by iteration we see that

$$
\frac{\mu\left(Q_{\rho}(y, s)\right)}{\mu\left(Q_{\rho / 2}(y, s)\right)} \frac{\mu\left(Q_{\rho / 2}(y, s)\right)}{\mu\left(Q_{\rho / 4}(y, s)\right)} \cdots \frac{\mu\left(Q_{\rho / 2^{N}}(y, s)\right)}{\mu\left(Q_{\rho / 2^{N+1}}(y, s)\right)}>\widetilde{K}^{N}
$$

or equivalently,

$$
\frac{\mu\left(Q_{\rho}(y, s)\right)}{\mu\left(Q_{\rho / 2^{N+1}}(y, s)\right)}>\widetilde{K}^{N}
$$

Doing a change of variables and manipulating the inequality we obtain

$$
\frac{\mu\left(Q_{2^{N+1} \rho}(y, s)\right)}{\rho^{n+1}}>\frac{\widetilde{K}^{N} \mu\left(Q_{\rho}(y, s)\right)}{\rho^{n+1}},
$$

for $2^{N+1} \rho=\rho_{0}$. Set $\rho_{N}=2^{-N-1} \rho_{0}$, so that $\rho_{N} \rightarrow 0$ is equivalent to $N \rightarrow \infty$. Hence

$$
\frac{\left(2^{n+1}\right)^{N+1}}{\widetilde{K}^{N}} \frac{\mu\left(Q_{\rho_{0}}(y, s)\right)}{\rho_{0}^{n+1}}>\frac{\mu\left(Q_{\rho_{N}}(y, s)\right)}{\rho_{N}^{n+1}},
$$

and therefore, since $\widetilde{K}>2^{n+1}$, we have that

$$
\lim _{\rho_{N} \rightarrow 0} \frac{\mu\left(Q_{\rho_{N}}(y, s)\right)}{\rho_{N}^{n+1}} \leq\left(\lim _{N \rightarrow \infty} \frac{\left(2^{n+1}\right)^{N+1}}{\widetilde{K}^{N}}\right)\left(\frac{\mu\left(Q_{\rho_{0}}(y, s)\right)}{\rho_{0}^{n+1}}\right) \rightarrow 0 .
$$

Since this is also true with $\rho_{0}$ replaced by any fixed $\rho_{1} \in\left[\frac{\rho_{0}}{2}, \rho_{0}\right]$, and $\rho_{N}=2^{-N-1} \rho_{1}$, we conclude that

$$
\lim _{\rho \rightarrow 0} \frac{\mu\left(Q_{\rho}(y, s)\right)}{\rho^{n+1}}=0
$$

for $(y, s) \in E$, where $E$ is a Borel measurable set with $\mu(E)>0$. We want to show that this cannot happen, and therefore we have (3.1.28).

Without loss of generality, we can assume that $E \subset Q_{0}$, where $Q_{0}$ is compact. Let $\epsilon>0$. By (3.1.29) and a covering lemma we obtain $\left\{Q_{5 \rho_{i}}\left(y_{i}, s_{i}\right)\right\}$ such that 
1. $E \subset \bigcup_{i} Q_{5 \rho_{i}}\left(y_{i}, s_{i}\right)$,

2. $Q_{\rho_{i}}\left(y_{i}, s_{i}\right) \cap Q_{\rho_{j}}\left(y_{j}, s_{j}\right)=\emptyset, i \neq j$,

3. $\mu\left(Q_{5 \rho_{i}}\left(y_{i}, s_{i}\right)\right) \leq \epsilon \rho_{i}^{n+1} \forall i$.

Therefore, by ADR and the above properties we have that

$$
\begin{aligned}
\mu(E) & \leq \sum_{i} \mu\left(Q_{5 \rho_{i}}\left(y_{i}, s_{i}\right)\right) \leq \epsilon \sum_{i} \rho_{i}^{n+1} \\
& \leq M_{0} \epsilon \sum \sigma\left(Q_{\rho_{i}}\left(y_{i}, s_{i}\right) \cap \Sigma\right) \leq M_{0} \epsilon \sigma\left(2 Q_{0} \cap \Sigma\right) .
\end{aligned}
$$

Letting $\epsilon \rightarrow 0$ we obtain a contradiction to our assumption that $\mu(E)>0$. This proves that we must have (3.1.28).

By the Lebesgue Besicovitch Differentiation Theorem, for $\mu$ a.e. $(y, s) \in F \subset \Delta_{r}(x, t)$, for $F$ Borel, we have

$$
\lim _{\rho \rightarrow 0} \frac{\mu\left(Q_{\rho}(y, s) \backslash F\right)}{\mu\left(Q_{\rho}(y, s)\right)}=0 .
$$

If $\mu$ were not absolutely continuous with respect to $\sigma$ on $\Delta_{r}(x, t)$, then for some $F$ Borel $\subset \Delta_{r}(x, t)$, we would have $\sigma(F)=0$ and $\mu(F)>0$. We will show that this cannot happen. Choose $(y, s) \in F$ such that (3.1.28) and (3.1.30) hold when $K=2^{n+2}$. Combining (3.1.25) with $P=Q_{2 \rho}(y, s) \backslash F$ and (3.1.28) we have that for some small $\rho>0$

$$
\begin{aligned}
\mu\left(Q_{\rho}(y, s)\right) & \leq \epsilon_{1} \mu\left(Q_{2 \rho}(y, s)\right)+c_{1} \mu\left(Q_{2 \rho}(y, s) \backslash F\right) \\
& \leq \epsilon_{1} 2^{n+2} \mu\left(Q_{\rho}(y, s)\right)+c_{1} \mu\left(Q_{2 \rho}(y, s) \backslash F\right) .
\end{aligned}
$$

Dividing the above inequality by $\mu\left(Q_{\rho}(y, s)\right)$, we see that

$$
1 \leq \epsilon_{1} 2^{n+2}+c_{1} \frac{\mu\left(Q_{2 \rho}(y, s) \backslash F\right)}{\mu\left(Q_{\rho}(y, s)\right)} \leq \epsilon_{1} 2^{n+2}+c_{1} 2^{n+2} \frac{\mu\left(Q_{2 \rho}(y, s) \backslash F\right)}{\mu\left(Q_{2 \rho}(y, s)\right)},
$$

by (3.1.28). Letting $\rho \rightarrow 0$ and using (3.1.30) we get a contradiction, provided $\epsilon_{1}<2^{-n-2}$. Hence $\mu<<\sigma$ on $\Delta_{r}(x, t)$ and by the Radon-Nikodym theorem $d \mu=g d \sigma$ on $\Delta_{r}(x, t)$. 
Continuing the proof of Lemma 3.1.24, we assume without loss of generality that $\mu\left(Q_{2 \rho}(y, s)\right) \neq 0$, since if $\mu\left(Q_{2 \rho}(y, s)\right)=0$, then trivially we have (3.1.27).

Let $d(Z, \tau)$ denote the parabolic distance from $(Z, \tau) \in Q_{2 \rho}(y, s)$ to $\partial Q_{2 \rho}(y, s)$, where $(y, s) \in \Sigma$. Define

$$
f(z, \tau)=\left(\frac{d(z, \tau)}{2 \rho}\right)^{n+1} g(z, \tau), \text { for }(z, \tau) \in \Delta_{2 \rho}(y, s)
$$

Then there exists a constant $C=C(n)$ and $c=c(n)$ such that

1. $C f(z, \tau) \geq g(z, \tau)$ whenever $(z, \tau) \in \Delta_{\rho}(y, s)$,

2. $f(z) \leq c g(z)$ whenever $(z, \tau) \in \Delta_{2 \rho}(y, s)$.

Let $(z, \tau) \in \Sigma$ and $\eta>0$ be such that $Q_{\eta}(z, \tau) \subset Q_{2 \rho}(y, s)$. Set

$$
f_{\Delta_{\eta}(z, \tau)} f d \sigma:=\frac{1}{\sigma\left(\Delta_{\eta}(z, \tau)\right)} \iint_{\Delta_{\eta}(z, \tau)} f d \sigma
$$

and let

$$
\lambda \geq C_{0} M_{0} \rho^{-n-1} \iint_{\Delta_{2 \rho}(y, s)} g d \sigma:=\lambda_{0},
$$

where $C_{0}=C_{0}(n)$ will be introduced momentarily, and $M_{0}$ is the ADR constant.

For $(z, \tau) \in \Delta_{2 \rho}(y, s)$, define $\widetilde{Q}:=Q\left((z, \tau), \frac{d(z, \tau)}{2}\right)$ and $\widetilde{\Delta}:=\widetilde{Q} \cap \Sigma$. For any $\left(z^{\prime}, \tau^{\prime}\right) \in \widetilde{Q}$, we see that $d\left(z^{\prime}, \tau^{\prime}\right) \approx d(z, \tau)$. Then $\widetilde{Q} \subset Q_{2 \rho}(y, s)$, and using ADR we obtain that

$$
\begin{aligned}
\int_{\widetilde{\Delta}} f d \sigma & =\frac{1}{\sigma(\widetilde{\Delta})} \iint_{\widetilde{\Delta}} f d \sigma \\
& \leq M_{0}\left(\frac{d(z, \tau)}{2}\right)^{-n-1} \iint_{\widetilde{\Delta}} f d \sigma \\
& =M_{0} d(z, \tau)^{-n-1} 2^{n+1} \iint_{\widetilde{\Delta}}\left(\frac{d\left(z^{\prime}, \tau^{\prime}\right)}{2 \rho}\right)^{n+1} g d \sigma \\
& \leq C_{0} M_{0} d(z, \tau)^{-n-1} \iint_{\widetilde{\Delta}}\left(\frac{d(z, \tau)}{\rho}\right)^{n+1} g d \sigma
\end{aligned}
$$




$$
\leq C_{0} M_{0} \rho^{-n-1} \iint_{\Delta_{2 \rho}(y, s)} g d \sigma=\lambda_{0}
$$

In other words, we have

$$
f f_{\widetilde{\Delta}} f d \sigma \leq \lambda_{0}
$$

From differentiation theory, we see that

$$
\lim _{\eta \rightarrow 0} f_{Q_{\eta}(z, \tau)} f d \sigma=f(z, \tau)
$$

for $\sigma$ a.e. $(z, \tau) \in \Delta_{2 \rho}(y, s)$.

Define

$$
F(\lambda):=\left\{(z, \tau) \in \Delta_{2 \rho}(y, s): f(z, \tau)>\lambda\right\}
$$

for $\lambda>0$ and let $(z, \tau) \in F(\lambda), \lambda \geq \lambda_{0}$, be a point where (3.1.33) holds. Then there exists $\eta_{0}=\eta_{0}(z, \tau) \leq \frac{d(z, \tau)}{2}$, small enough, such that if $\tilde{\eta}<\eta_{0}$,

$$
f_{\Delta_{\tilde{\eta}}(z, \tau)} f d \sigma>\lambda \quad \text { and } \quad f_{\Delta_{\eta_{0}}(z, \tau)} f d \sigma=\lambda .
$$

Choose $\eta=\eta(z, \tau):=\frac{1}{5} \eta_{0}$. Then for $0<5 \eta \leq \frac{d(z, \tau)}{2}$ the previous fact may be reformulated as follows:

$$
f_{\Delta_{5_{\eta}}(z, \tau)} f d \sigma=\lambda, \quad \text { and } \quad \oiiint_{\Delta_{\eta^{\prime}}(z, \tau)} f d \sigma>\lambda \text {, }
$$

for $0<\eta^{\prime} \leq 5 \eta$. Combining the above inequalities we obtain that

$$
\oiiint_{\Delta_{5 \eta}(z, \tau)} f d \sigma \leq f f_{\Delta_{\eta^{\prime}}(z, \tau)} f d \sigma,
$$

for $0<\eta^{\prime} \leq 5 \eta$. Equivalently, by definition of $f$, canceling out $(2 \rho)^{-n-1}$ on both sides of the inequality, and using the fact that $d(z, \tau) \approx d\left(z^{\prime}, \tau^{\prime}\right)$ for $\left(z^{\prime}, \tau^{\prime}\right)$ in $\widetilde{Q}$, and that $Q_{\eta^{\prime}}(z, \tau) \subset$ $Q_{5 \eta}(z, \tau) \subset \widetilde{Q}$, we obtain 


$$
\frac{d(z, \tau)^{n+1}}{\sigma\left(Q_{5 \eta}(z, \tau) \cap \Sigma\right)} \iint_{\Delta_{5 \eta}(z, \tau)} g d \sigma \lesssim \frac{d(z, \tau)^{n+1}}{\sigma\left(\Delta_{\eta^{\prime}}(z, \tau)\right)} \iint_{\Delta_{\eta^{\prime}}(z, \tau)} g d \sigma .
$$

By ADR, (3.1.36) becomes

$$
M_{0}^{-1}(5 \eta)^{-n-1} \iint_{\Delta_{5 \eta}(z, \tau)} g d \sigma \lesssim M_{0}\left(\eta^{\prime}\right)^{-n-1} \iint_{\Delta_{\eta^{\prime}}(z, \tau)} g d \sigma .
$$

Let $\frac{\eta}{2} \leq \eta^{\prime} \leq 5 \eta<\frac{d(z, \tau)}{2}$. Then (3.1.37) becomes

$$
M_{0}^{-1} \iint_{\Delta_{5 \eta}(z, \tau)} d \mu \lesssim M_{0} \iint_{\Delta_{\eta^{\prime}}(z, \tau)} d \mu
$$

since $g d \sigma=d \mu$ on $\Delta_{r}(x, t)$. Equivalently, for $\frac{\eta}{2} \leq \eta^{\prime} \leq 5 \eta$,

$$
\mu\left(Q_{5 \eta}(z, \tau)\right) \lesssim M_{0}^{2} \mu\left(Q_{\eta^{\prime}}(z, \tau)\right) .
$$

Setting $\eta^{\prime}=\frac{\eta}{2}$ and using monotonicity, we find that (3.1.38) implies

$$
\mu\left(Q_{\eta}(z, \tau)\right) \leq \mu\left(Q_{5 \eta}(z, \tau)\right) \lesssim M_{0}^{2} \mu\left(Q_{\eta / 2}(z, \tau)\right)
$$

Set

$$
E(\lambda):=\left\{(w, \xi) \in \Delta_{2 \rho}(y, s): f(w, \xi) \leq \lambda\right\}
$$

for $\lambda>0$. Suppose for a given $\delta>0$, small enough, that

$$
\sigma\left(E(\delta \lambda) \cap Q_{\eta}(z, \tau)\right) \geq(1-\zeta) \sigma\left(Q_{\eta}(z, \tau)\right)
$$

where $\zeta$ is as in (3.1.26). Then from (3.1.39) and (3.1.25) with $(y, s), 2 \rho$, and $P$ replaced by $(z, \tau), \eta$, and $E(\delta \lambda) \cap Q_{\eta}(z, \tau)$, respectively, we see that if $\lambda \geq \lambda_{0}$ and $0<\epsilon_{1} \leq M^{-1} M_{0}^{-2}$, with $M$ a sufficiently large constant to be chosen momentarily, then

$$
\begin{aligned}
\mu\left(Q_{\eta / 2}(z, \tau)\right) & \leq \epsilon_{1} \mu\left(Q_{\eta}(z, \tau)\right)+C_{1} \mu\left(E(\delta \lambda) \cap Q_{\eta}(z, \tau)\right) \\
& \leq C \epsilon_{1} M_{0}^{2} \mu\left(Q_{\eta / 2}(z, \tau)\right)+C_{1} \mu\left(E(\delta \lambda) \cap Q_{\eta}(z, \tau)\right)
\end{aligned}
$$




$$
\leq \frac{1}{2} \mu\left(Q_{\eta / 2}(z, \tau)\right)+C_{1} \mu\left(E(\delta \lambda) \cap Q_{\eta}(z, \tau)\right)
$$

by the choice $M:=1 /(2 C)$. Hiding the small term on the left hand side of the inequality, we obtain

$$
\mu\left(Q_{\eta / 2}(z, \tau)\right) \leq 2 C_{1} \mu\left(E(\delta \lambda) \cap Q_{\eta}(z, \tau)\right)
$$

Recall that $d(z, \tau) \approx d\left(z^{\prime}, \tau^{\prime}\right)$ for $\left(z^{\prime}, \tau^{\prime}\right)$ in $\widetilde{Q}$, and that $Q_{\eta}(z, \tau) \subset Q_{5 \eta}(z, \tau) \subset \widetilde{Q}$. Multiplying (3.1.42) by $(d(z, \tau) / 2 \rho)^{n+1}\left(\sigma\left(\Delta_{\eta}(z, \tau)\right)\right)^{-1}$, we find that

$$
\frac{1}{\sigma\left(\Delta_{\eta}(z, \tau)\right)} \iint_{\Delta_{\eta / 2}(z, \tau)} f d \sigma \lesssim \frac{1}{\sigma\left(\Delta_{\eta}(z, \tau)\right)} \iint_{\Delta_{\eta}(z, \tau) \cap E(\delta \lambda)} f d \sigma .
$$

Thus, by ADR, the definition of $E(\delta \lambda)$, and (3.1.35), with $\eta^{\prime}=\eta / 2$, we have

$$
\lambda \leq \oiiint_{\Delta_{\eta / 2}(z, \tau)} f d \sigma \lesssim_{M_{0}} \lambda \delta
$$

We have therefore shown that if (3.1.41) holds for a given $\delta>0$, then

$$
\delta \geq C\left(M_{0}\right)^{-1}
$$

Consequently, (3.1.41) fails for $\delta=\delta_{0}=\frac{1}{2 C\left(M_{0}\right)}$. Therefore by the definitions (3.1.34) and (3.1.40),

$$
\sigma\left(F\left(\delta_{0} \lambda\right) \cap Q_{\eta}(z, \tau)\right) \geq \zeta \sigma\left(\Delta_{\eta}(z, \tau)\right)
$$

Using a standard covering argument, we find a sequence $\left\{\left(\eta_{i}, z_{i}, \tau_{i}\right)\right\}$ for which (3.1.35) and (3.1.43) hold with $\eta=\eta_{i},(z, \tau)=\left(z_{i}, \tau_{i}\right)$, and also such that

1. $F(\lambda) \subset \bigcup_{i} Q_{5 \eta_{i}}\left(z_{i}, \tau_{i}\right)$, up to a set of $\sigma$-measure zero,

2. $Q_{\eta_{i}}\left(z_{i}, \tau_{i}\right) \cap Q_{\eta_{j}}\left(z_{j}, \tau_{j}\right)=\emptyset, i \neq j$.

Then

$$
\iint_{F(\lambda)} f d \sigma \leq \sum_{i} \iint_{\Delta_{5 \eta_{i}}\left(z_{i}, \tau_{i}\right)} f d \sigma
$$




$$
\begin{aligned}
& =\sum_{i} \sigma\left(\Delta_{5 \eta_{i}}\left(z_{i}, \tau_{i}\right)\right) f f_{\Delta_{5 \eta_{i}}\left(z_{i}, \tau_{i}\right)} f d \sigma \\
& \stackrel{(3.1 .35)}{=} \sum_{i} \lambda \sigma\left(\Delta_{5 \eta_{i}}\left(z_{i}, \tau_{i}\right)\right) \\
& \stackrel{(\mathrm{ADR})}{\lesssim} \sum_{i} \lambda \sigma\left(\Delta_{\eta_{i}}\left(z_{i}, \tau_{i}\right)\right) \\
& \stackrel{(3.1 .43)}{\lesssim} \zeta^{-1} \lambda \sum_{i} \sigma\left(F\left(\delta_{0} \lambda\right) \cap Q_{\eta_{i}}\left(z_{i}, \tau_{i}\right)\right) \\
& \lesssim \zeta^{-1} \lambda \sigma\left(F\left(\delta_{0} \lambda\right)\right) .
\end{aligned}
$$

Let $A:=M \zeta^{-1}$, where $M$ is the implicit constant in the last inequality, so that, uniformly in $\lambda$ for $\lambda \geq \lambda_{0}$, we have

$$
\iint_{F(\lambda)} f d \sigma \leq A \lambda \sigma\left(F\left(\delta_{0} \lambda\right)\right)
$$

For a large positive integer $m$ set

$$
\begin{aligned}
f_{m} & =\min \{f, m\} \\
F_{m}(\lambda) & =\left\{(z, \tau) \in \Delta_{2 \rho}(y, s): f_{m}(z, \tau)>\lambda\right\} .
\end{aligned}
$$

Then $F_{m}(\lambda)=F(\lambda)$, and $F_{m}\left(\delta_{0} \lambda\right)=F\left(\delta_{0} \lambda\right)$, provided that $m>\lambda \geq \lambda_{0}$, and of course, $F_{m}(\lambda)=\emptyset$ if $m \leq \lambda$. Thus, (3.1.44) holds with $f_{m}, F_{m}$ in place of $f, F$, i.e.,

$$
\iint_{F_{m}(\lambda)} f_{m} d \sigma \leq A \lambda \sigma\left(F_{m}\left(\delta_{0} \lambda\right)\right) \leq 2^{n+1} M_{0} A \lambda \rho^{n+1},
$$

where the last inequality follows trivially from $\mathrm{ADR}$, since $F_{m}(\lambda) \subset \Delta_{2 \rho}(y, s)$ for each $\lambda>0$.

Using a Lebesgue-Stieltjes integral with measure $f_{m} d \sigma$, for $m>\lambda_{0}$, we then obtain that

$$
\begin{gathered}
\iint_{F_{m}\left(\lambda_{0}\right)} f_{m}^{1+\gamma} d \sigma=\gamma \int_{\lambda_{0}}^{\infty} \lambda^{\gamma-1}\left(\iint_{F_{m}(\lambda)} f_{m} d \sigma\right) d \lambda+\lambda_{0}^{\gamma} \iint_{F_{m}\left(\lambda_{0}\right)} f_{m} d \sigma \\
\stackrel{(3.1 .45)}{\leq} A \gamma \int_{\lambda_{0}}^{\infty} \lambda^{\gamma} \sigma\left(F_{m}\left(\delta_{0} \lambda\right)\right) d \lambda+C A \lambda_{0}^{\gamma+1} \rho^{n+1}
\end{gathered}
$$




$$
\leq A \gamma(1+\gamma)^{-1} \delta_{0}^{-1-\gamma} \iint_{\Delta_{2 \rho}(y, s)} f_{m}^{1+\gamma} d \sigma+C A \lambda_{0}^{\gamma+1} \rho^{n+1},
$$

where in the last step we have extended the integral in $\lambda$ to the interval $(0, \infty)$, and used the definition of $F_{m}$ and the change of variable $\lambda \rightarrow \delta_{0}^{-1} \lambda$.

We now choose $\gamma$ so that

$$
\frac{A \gamma}{(1+\gamma) \delta_{0}^{1+\gamma}} \leq \frac{1}{2} .
$$

Then from the above inequality, we see that

$$
\iint_{\Delta_{2 \rho}(y, s)} f_{m}^{1+\gamma} d \sigma \leq \frac{1}{2} \iint_{\Delta_{2 \rho}(y, s)} f_{m}^{1+\gamma} d \sigma+C A \lambda_{0}^{\gamma+1} \rho^{n+1} .
$$

Therefore, for some large $C^{\prime}=C^{\prime}\left(M_{0}, \zeta, c_{1}, n\right)$, by definition of $\lambda_{0}$ (3.1.31), we have

$$
\iint_{\Delta_{2 \rho}(y, s)} f_{m}^{1+\gamma} d \sigma \leq C^{\prime} \rho^{(-n-1) \gamma}\left(\iint_{\Delta_{2 \rho}(y, s)} g d \sigma\right)^{1+\gamma},
$$

uniformly in $m$. Let $m \rightarrow \infty$ and use the monotone convergence theorem to conclude that

$$
\iint_{\Delta_{2 \rho}(y, s)} f^{1+\gamma} d \sigma \leq C^{\prime} \rho^{(-n-1) \gamma}\left(\iint_{\Delta_{2 \rho}(y, s)} g d \sigma\right)^{1+\gamma} .
$$

Then since $g \lesssim f$ on $\Delta_{p}(y, s),(3.1 .46)$ becomes

$$
\iint_{\Delta_{\rho}(y, s)} g^{1+\gamma} d \sigma \leq C \rho^{(-n-1) \gamma}\left(\iint_{\Delta_{2 \rho}(y, s)} g d \sigma\right)^{1+\gamma},
$$

proving Lemma 3.1.24.

Proof of Theorem 3.1.3. Fix $Q_{r}\left(x_{0}, t_{0}\right)$ with $\left(x_{0}, t_{0}\right) \in \Sigma, r>0$, and let $(Y, s) \in \Omega \backslash Q_{4 r}\left(x_{0}, t_{0}\right)$. Then for all $Q_{\rho}(z, \tau)$ such that $Q_{2 \rho}(z, \tau) \subset Q_{r}\left(x_{0}, t_{0}\right)$ we see that $(Y, s) \in \Omega \backslash Q_{4 \rho}(z, \tau)$. Therefore Lemma 3.1.5 applies in each such $Q_{\rho}(z, \tau)$ and if we set $\mu:=\omega^{Y, s}$, then $\mu$ satisfies Lemma 3.1.24 with $C_{1}=C\left(\epsilon_{1}\right)$ and $\zeta=\eta\left(\epsilon_{1}\right)$ (here we are using Lemma 2.0.1). Applying Lemma 3.1.24 we obtain Theorem 3.1.3. 


\subsubsection{Applications}

We discuss two applications of Theorem 3.1.3. The first is an extension of a result proved in $[\mathrm{NS}]$. We refer to [NS] for definitions of the terms not previously defined. The second application will be stated in this section, and the details of the proof can be found in Section 3.3 of this paper.

Theorem 3.1.47. Let $\Omega \subset \mathbb{R}^{n+1}$ satisfy a time-synchronized two cubes condition, and suppose that $\Sigma$ is parabolic uniformly rectifiable (in particular $\Sigma$ is globally ADR). Then caloric measure $\omega$ satisfies a local weak- $A_{\infty}$ condition with respect to $\sigma$, equivalently, $\omega \ll \sigma$ and the Radon-Nikodym derivative d $\omega / d \sigma$ verifies the weak Reverse Hölder condition (1.2.39).

A few remarks are in order. In [NS], the authors obtain a similar result, but assuming in addition that $\Omega$ satisfies a parabolic Harnack Chain condition. Our Theorem 3.1.3 allows us to dispense with the latter connectivity assumption in Theorem 3.1.47. The conclusion in [NS] is that $\omega$ satisfies an $A_{\infty}$ condition (which entails doubling) with respect to $\sigma$, but in the absence of connectivity the non-doubling weak- $A_{\infty} /$ weak Reverse Hölder conclusion is best possible. We remark that the aforementioned result of [NS], and our Theorem 3.1.47, are the parabolic analogues of results proved in $[\mathrm{DJ}]$ and in $[\mathrm{BL}]$, respectively.

The hypotheses of the theorem correspond to the case $T_{\min }=-\infty, T_{\max }=\infty$. A sketch of the proof is as follows. One first invokes the deep fact proved in [NS, Theorem 1.2] that under the hypotheses of Theorem 3.1.47, one obtains an interior "big pieces" approximation (see [NS] for the precise definition), analogous to that proved in the elliptic setting in [DJ], by domains of the sort considered in [LM]. By the result of [LM], plus a standard maximum principle argument, one obtains the $(\theta, \beta)$-local ampleness condition 3.1.2. In addition, it is not difficult to show that in the presence of ADR, the time-synchronized 
two cubes condition of [NS] implies global time-backwards ADR (in fact, it implies timesymmetric ADR). At this point, the conclusion follows by Theorem 3.1.3.

Theorem 3.1.48. Let $\Omega \subset \mathbb{R}^{n+1}$, whose quasi-lateral boundary $\Sigma$ is globally ADR and timebackwards ADR. Suppose that the Dirichlet problem is "BMO-solvable” in $\Omega$. Then caloric measure $\omega$ satisfies a local weak- $A_{\infty}$ condition with respect to $\sigma$, equivalently, $\omega \ll \sigma$ and the Radon-Nikodym derivative $d \omega / d \sigma$ verifies the weak Reverse Hölder condition (1.2.39).

The result is a parabolic version of the main theorem of [HLe], which in turn entailed removing all connectivity hypotheses (in particular, the Harnack Chain condition), from earlier elliptic results of $[\mathrm{DKP}]$ and $[\mathrm{Z}]$. See Section 3.3 for the proof, and for precise definitions of the relevant terminology.

\section{2 $L^{p}$ solvability}

\subsubsection{Introduction}

In less austere settings, say in Lipschitz cylinders or even $\operatorname{Lip}(1,1 / 2)$ domains, the equivalence between $L^{p}$ solvability of the initial-Dirichlet problem and the $A_{\infty}$ property of $\omega$, is well-known (see, e.g., [N, Theorem 4.3]: for such a domain $\Omega$, with $T_{\min }(\Omega)>-\infty$, one may consider the initial-Dirichlet problem in $\Omega=\Omega^{T}, T=T_{\min }(\Omega)$, and then prove the main implication (1) implies (2) (or something essentially equivalent, namely that $N_{*} u \approx$ $\mathcal{M}_{\omega} f$, where $\mathcal{M}_{\omega}$ is the Hardy-Littlewood maximal operator with respect to parabolic measure at some fixed pole) either by using Harnack's inequality and the Harnack chain property to move from an arbitrary point in a non-tangential "cone" to a time forward point, or by extending backwards in time, either by even reflection of the domain across the hyperplane $\left\{t \equiv T_{m i n}\right\}$, or simply by extending the time-slice $\Omega_{T_{\min }}$ backwards in time as a cylinder. In the more general setting considered here, neither of these technical devices 
is available, and that is why we work in an ambient domain $\Omega$, and then solve the initialDirichlet problem in subdomains $\Omega^{T}$ with $T-T_{\min } \gtrsim R_{0}^{2}$, where $R_{0}:=\operatorname{diam}(\Sigma)$.)

\subsubsection{Main Result}

Let us make the following geometric observation. Set $R_{0}:=\operatorname{diam}(\Sigma)$. Then there is a constant $c$, depending only on dimension and ADR, such that $T_{\max }-T_{\min } \geq c R_{0}^{2}$. Indeed, suppose first that $R_{0}<\infty$, and set $r^{2}:=T_{\max }-T_{\min }$. Then $\Sigma$ is contained in a closed rectangle in $\mathbb{R}^{n+1}$ with dimensions $R_{0} \times \ldots \times R_{0} \times r^{2}$, of volume $\left(R_{0}\right)^{n} r^{2}$. We may then cover $\Sigma$ by a collection of parabolic cubes $\left\{Q_{r}^{i}\right\}_{i}$, centered on $\Sigma$, of cardinality at most $C\left(R_{0} / r\right)^{n}$. By the ADR property, since $\Sigma$ has diameter $R_{0}$, we have

$$
\left(R_{0}\right)^{n+1} \lesssim \sigma(\Sigma) \leq \sum_{i} \sigma\left(\Sigma \cap Q_{r}^{i}\right) \lesssim\left(\frac{R_{0}}{r}\right)^{n} r^{n+1}
$$

Thus $r \gtrsim R_{0}$, as claimed.

Next, suppose that $R_{0}=\infty$, but that $T_{\max }-T_{\min }=: r^{2}<\infty$. Then for any fixed $\left(x_{0}, t_{0}\right) \in \Sigma$, and any $R \in(r, \infty)$, the surface cube $\Delta_{R}\left(x_{0}, t_{0}\right):=Q_{R}\left(x_{0}, t_{0}\right) \cap \Sigma$ is contained in a rectangle with dimensions $R \times \ldots \times R \times r^{2}$, and volume $R^{n} r^{2}$. We may then repeat the previous argument to see that $r \gtrsim R$, and then let $R \rightarrow \infty$.

In the sequel, we shall continue to use the notation $R_{0}:=\operatorname{diam}(\Sigma)$.

We now formulate the equivalence between the weak- $A_{\infty}$ property of parabolic measure, and $L^{p}$ solvability of the initial-Dirichlet problem.

Theorem 3.2.1. Let $L$ be a divergence form parabolic operator defined on $\Omega$. Suppose that $\Sigma$ is time-backwards $A D R$, and assume further that if $R_{0}=\infty$, then $T_{\min }=-\infty$. Then TFAE:

(1) For every $\kappa_{0} \in(0,1)$, there is an exponent $q>1$, possibly depending on $\kappa_{0}$, such that 
$\omega^{Y, s} \ll \sigma$, and $k^{Y, s}:=d \omega^{Y, s} / d \sigma$ satisfies the reverse Hölder estimate

$$
\left(f_{\Delta}\left(k^{Y, s}\right)^{q} d \sigma\right)^{1 / q} \lesssim_{\kappa_{0}} f_{2 \Delta} k^{Y, s} d \sigma \approx \frac{\omega^{Y, s}(2 \Delta)}{\sigma(\Delta)},
$$

on every $\Delta=\Sigma \cap Q_{r}\left(x_{0}, t_{0}\right)$, with $t_{0}-T_{\text {min }} \geq \kappa_{0} R_{0}^{2}$ and $r<\sqrt{\kappa_{0}} R_{0} / 2$, and for all $(Y, s) \in \Omega \backslash Q_{4 r}\left(x_{0}, t_{0}\right)$, uniformly for all such $\Delta$ and $(Y, s)$.

(2) For every $\kappa_{1} \in(0,1)$, there is an exponent $p<\infty$, possibly depending on $\kappa_{1}$, such that if $T_{0}-T_{\min } \geq \kappa_{1} R_{0}^{2}$, and $f \in C_{c}(\Sigma)$ with compact support in $\Sigma^{T_{0}}$, then the parabolic measure solution (i.e., Perron solution) $u$ of the initial-Dirichlet (resp., Dirichlet) problem for L in $\Omega=\Omega^{T}$ with $T=T_{\min }>-\infty$ (resp., in $\Omega$ if $\left.T=-\infty\right)$, with lateral data $f$, satisfies for all $(x, t) \in \Sigma^{T_{0}}$,

$$
N_{*} u(x, t) \lesssim_{\kappa_{1}}\left(\mathcal{M}\left(|f|^{p}\right)(x, t)\right)^{1 / p}
$$

where $\mathcal{M}$ denotes the parabolic Hardy-Littlewood maximal operator on $\Sigma$.

(3) For every $\kappa_{1} \in(0,1)$, there is an exponent $p<\infty$, possibly depending on $\kappa_{1}$, such that if $T_{0}-T_{\min } \geq \kappa_{1} R_{0}^{2}$, then the initial-Dirichlet problem for $L$ is $L^{p}$ solvable in $\Omega^{T_{0}}$, with the estimate $\left\|N_{*} u\right\|_{L^{p}(\Sigma)} \lesssim_{\kappa_{1}}\|f\|_{L^{p}(\Sigma)}$.

Furthermore, for appropriately related $\kappa_{0}, \kappa_{1}$, the exponents $q$ in (1), and $p$ in (2) and (3), satisfy the duality relationship $p=q /(q-1)$.

Here $N_{*} u$ denotes the non-tangential maximal function, of course taken with respect to parabolic cones.

We note that we are implicitly assuming here, as above, that the continuous Dirichlet problem is solvable for $L$; we know that this is true if $L$ is the heat operator: see Remarks 1.2.21 and 1.2.23. In either case, the associated caloric/parabolic measure $\omega=\omega_{L}$ exists. 
We mention that in Theorem 3.2.1, we address only the issue of $L^{p}$ solvability, i.e, existence of solutions with $L^{p}$ estimates; we do not discuss the question of uniqueness.

Proof of Theorem 3.2.1. The main step in the proof is to show that (1) implies (2). We turn our attention to this matter first.

For convenience, we shall treat the case $T_{\min }>-\infty$; the proof in the case $T_{\min }=-\infty$ is similar, but slightly simpler, and we leave the details to the interested reader.

(1) implies (2). Recall that $R_{0}:=\operatorname{diam}(\Sigma) \in(0, \infty]$. We assume that for every $\kappa_{0} \in(0,1)$, and for each $\Delta=Q_{r}\left(x_{0}, t_{0}\right) \cap \Sigma$, with $\left(x_{0}, t_{0}\right) \in \Sigma, t_{0}-T_{\min } \geq \kappa_{0} R_{0}^{2}$ and $r<\sqrt{\kappa_{0}} R_{0} / 2$, and for all $(Y, s) \in \Omega \backslash Q_{4 r}\left(x_{0}, t_{0}\right)$, we have $\omega^{Y, s} \ll \sigma$ in $\Delta$, and there exists some $q>1$ such that $k^{Y, s} \in$ weak- $R H_{q}(\Delta)$, with uniform constants, i.e. (3.2.2) holds for the Radon-Nikodym derivative $k^{Y, s}:=d \omega^{Y, s} / d \sigma$, with $q$ and the implicit constants independent of $\Delta$ and $(Y, s)$; equivalently, $\omega^{Y, s} \in$ weak- $A_{\infty}(\Delta)$, with uniform control of the constants. Let $\kappa_{1} \in(0,1)$, and set $\kappa_{0}:=\kappa_{1} / 100$. Suppose that $T_{0}-T_{\min } \geq \kappa_{1} R_{0}^{2}=100 \kappa_{0} R_{0}^{2}$. Let $f$ be continuous on $\partial_{e} \Omega$, with compact support in $\Sigma^{T_{0}}$, and let $u$ be the parabolic measure solution of the continuous Dirichlet problem (see Definition 1.2.13) for $L$ with data $f$, in $\Omega$.

Our goal is to show that for $p=q /(q-1)$, and for all $(x, t) \in \Sigma^{T_{0}}$,

$$
N_{*} u(x, t) \lesssim\left(\mathcal{M}\left(|f|^{p}\right)(x, t)\right)^{1 / p},
$$

where $\mathcal{M}$ denotes the parabolic Hardy-Littlewood maximal operator on $\Sigma$, and hence that (1) implies (2).

To this end, we begin by defining non-tangential "cones" and maximal functions, as follows. First, we fix a collection of parabolic closed Whitney cubes covering $\Omega$, and we denote this collection by $\mathcal{W}$. We also fix a constant $v>0$ small enough so that, for each Whitney cube $I$, its concentric parabolic dilate $I^{*}:=(1+v) I$ also satisfies the Whitney 
properties. We will denote the collection of analogously fattened Whitney cubes $\mathcal{W}^{*}$.

Given $(x, t) \in \Sigma$, set

$$
\mathcal{W}(x, t):=\left\{I \in \mathcal{W}: \operatorname{diam}(I)<10 R_{0} \text { and } \operatorname{dist}((x, t), I) \leq 100 \operatorname{diam}(I)\right\}
$$

and define the (possibly disconnected) non-tangential "cone" with vertex $(x, t)$ by

$$
\Upsilon(x, t):=\operatorname{int}\left(\bigcup_{I \in \mathcal{W}(x, t)} I^{*}\right),
$$

where $\operatorname{int}(A)$ denotes the interior of the set $A$. For a continuous $u$ defined on $\Omega$, the nontangential maximal function of $u$ is defined by

$$
N_{*} u(x, t):=\sup _{(Y, s) \in \Upsilon(x, t)}|u(Y, s)|
$$

We now turn to the proof of (3.2.4).

Splitting $f$ into its positive and negative parts, we may suppose without loss of generality that $f \geq 0$, hence also $u \geq 0$. Let $(x, t) \in \Sigma$ and fix $\left(Y_{0}, s_{0}\right) \in \Upsilon(x, t)$. Then $\left(Y_{0}, s_{0}\right) \in I_{0}^{*}$, for some $I_{0} \in \mathcal{W}(x, t)$ such that

$$
r:=\delta\left(Y_{0}, s_{0}\right) \approx \operatorname{diam}\left(I_{0}\right) \approx\left\|(x, t)-\left(Y_{0}, s_{0}\right)\right\| .
$$

Of course, by definition of $\mathcal{W}(x, t)$ we have $r<K R_{0}$, for some sufficiently large universal constant $K$.

Let

$$
Q_{0}:=Q((x, t), r), \quad Q_{k}:=2^{k} Q_{0}=Q\left((x, t), 2^{k} r\right), \quad k=1,2,3 \ldots,
$$

and define corresponding surface cubes and subdomains:

$$
\Delta_{k}:=Q_{k} \cap \Sigma, \quad \Omega_{k}:=Q_{k} \cap \Omega, \quad k \geq 0 .
$$


Define a continuous partition of unity $\sum_{k \geq 0} \varphi_{k} \equiv 1$ on $\Sigma$, such that $0 \leq \varphi_{k} \leq 1$ for all $k \geq 0$, with

$$
\operatorname{supp}\left(\varphi_{0}\right) \subset \Delta_{2}=: \mathcal{R}_{0}, \quad \operatorname{supp}\left(\varphi_{k}\right) \subset \mathcal{R}_{k}:=\Delta_{k+2} \backslash \Delta_{k}, k \geq 1
$$

Set $f_{k}:=f \varphi_{k}$, and let $u_{k}$ be the solution of the initial-Dirichlet problem in $\Omega^{T}=\Omega, T=$ $T_{\min }$, with initial data (at time $t=T_{\min }$ ) equal to zero, and data $f_{k}$ on $\Sigma$. Observe that since we are treating the case $T_{\min }>-\infty$, and hence by assumption $\operatorname{diam}(\Sigma)=R_{0}<\infty$, the boundary annulus $\mathcal{R}_{k}, k \geq 1$, is empty if $2^{k} r>R_{0}$; for such $k$, we have that $f_{k}$, and hence $u_{k}$, are identically zero. Thus, we may restrict our attention to those $k$ for which $2^{k} r \leq R_{0}$, so that $u=\sum_{0 \leq k \leq \log _{2}\left(R_{0} / r\right)} u_{k}$ in $\Omega$ (it may happen that $r>R_{0}$, but in that case only the term $k=0$ appears in the sum, and the following proof may be simplified considerably; we omit the very routine details).

Let us first observe that for each $k \geq 0$, and $(Y, s) \in \Omega$,

$$
u_{k}(Y, s)=\iint_{\mathcal{R}_{k} \cap\left\{T_{0}<\tau\right\}} f_{k}(z, \tau) d \omega^{Y, s}(z, \tau) .
$$

Indeed, since $f$ is compactly supported in $\Sigma^{T_{0}}$, it follows that $u_{k}$ is zero for $s \leq T_{0}$.

We now fix a sufficiently large integer $N$ to be chosen momentarily, and we claim that

$$
\sum_{k=0}^{N} u_{k}\left(Y_{0}, s_{0}\right) \lesssim_{N}\left(\mathcal{M}\left(f^{p}\right)(x, t)\right)^{1 / p} .
$$

To see this, we begin by recalling that by assumption, $T_{0}-T_{\min } \geq 100 \kappa_{0} R_{0}^{2}$, and by construction, $r<K R_{0}$, for a suitably large universal constant $K$. We may therefore cover $Q_{N+2} \cap\left\{(X, \tau): T_{0}<\tau\right\}$ by a collection $\mathcal{F}_{0}$ of pairwise disjoint half-open parabolic cubes of parabolic sidelength $b r$, where $b$ is a sufficiently small number to be chosen momentarily, in particular with $0<b \leq \sqrt{\kappa_{0}} /(100 K)$.

Let $\mathcal{F}:=\left\{Q \in \mathcal{F}_{0}: Q\right.$ meets $\left.\Sigma\right\}$, and for each $Q \in \mathcal{F}$, let $Q_{*}$ be a cube centered at $\left(x_{*}, t_{*}\right) \in \Sigma$, containing $Q$, of parabolic sidelength $\ell\left(Q_{*}\right)=5 \ell(Q)=5 b r$, so that $2 Q_{*} \subset$ 
$100 Q$. Then for $b$ suitably small, $\left(Y_{0}, s_{0}\right) \in \Omega \backslash 4 Q_{*}$ by (3.2.8). Thus, setting $\Delta_{*}:=Q_{*} \cap \Sigma$, we note that by hypothesis, we may apply the reverse Hölder estimate (3.2.2) with $\Delta=\Delta_{*}$, and with $(Y, s)=\left(Y_{0}, s_{0}\right)$. Let $\mathcal{F}_{*}$ denote the collection of all such $Q_{*}$.

Since $\sum_{k=0}^{N} f_{k}$ is supported in $\Delta_{N+2}=Q_{N+2} \cap \Sigma$, we then have that

$$
\begin{aligned}
\sum_{k=0}^{N} u_{k}\left(Y_{0}, s_{0}\right) \leq & \sum_{Q_{*} \in \mathcal{F}_{*}} \iint_{\Delta_{*}} f(z, \tau) d \omega^{Y_{0}, s_{0}}(z, \tau) \\
& \leq \sum_{Q_{*} \in \mathcal{F}_{*}} \sigma\left(\Delta_{*}\right)\left(\oiiint_{\Delta_{*}} f^{p} d \sigma\right)^{1 / p}\left(\oiiint_{\Delta_{*}}\left(k^{Y_{0}, s_{0}}\right)^{q} d \sigma\right)^{1 / q} \\
& \lesssim \sum_{Q_{*} \in \mathcal{F}_{*}}\left(\oiiint_{\Delta_{*}} f^{p} d \sigma\right)^{1 / p},
\end{aligned}
$$

where in the last step we have used the fact noted above: that the weak reverse Hölder estimate (3.2.2) may be applied to each $\Delta_{*}$ uniformly. Now by the ADR property, $\sigma\left(\Delta_{N+3}\right) \approx$ $\sigma\left(\Delta_{N+2}\right) \approx_{N, n, K, k_{0}} \sigma\left(\Delta_{*}\right)$, and by construction, we may suppose that each $\Delta_{*}$ is contained in $\Delta_{N+3}$. Consequently, for each $\Delta_{*}$ we have

$$
\oiiint_{\Delta_{*}} f^{p} d \sigma \lesssim_{n, K, \kappa_{0}} \oiiint_{\Delta_{N+3}} f^{p} d \sigma \lesssim_{n, K, \kappa_{0}} \mathcal{M}\left(f^{p}\right)(x, t) .
$$

Since $\operatorname{card}\left(\mathcal{F}_{*}\right) \leq C(N, n, b)$, with $b$ in turn depending only on $\kappa_{0}$ and $K$, the claimed bound (3.2.12) now follows.

Next, we claim that for $k \geq N+1$, with $N$ chosen large enough,

$$
u_{k}\left(Y_{0}, s_{0}\right) \lesssim 2^{-k \alpha}\left(\mathcal{M}\left(f^{p}\right)(x, t)\right)^{1 / p},
$$

from which the desired bound (3.2.4) follows immediately, since $\left(Y_{0}, s_{0}\right)$ is an arbitrary point in $\Upsilon(x, t)$.

Recall that $\Omega_{k}$ is defined in (3.2.9). We now fix $N$, depending only on the implicit constants in (3.2.8), such that $\left(Y_{0}, s_{0}\right) \in \Omega_{N-1}$. Having fixed $N$, we will allow implicit and generic constants to depend on $N$ without noting such dependence explicitly. 
For $k \geq N+1$, set

$$
\mathcal{W}_{k}:=\left\{I \in \mathcal{W}: I \text { meets } Q_{k-N}\right\}
$$

Note that for $N$ chosen large enough, depending only on the Whitney construction, we have that

$$
\bigcup_{I \in \mathcal{W}_{k}} I^{*} \subset Q_{k-3} \cap \Omega .
$$

Since $f_{k}$ vanishes in $\Delta_{k}$, by Lemma 2.0.21, we have

$$
u_{k}\left(Y_{0}, s_{0}\right) \lesssim 2^{-k \alpha}\left(2^{k} r\right)^{-n-2} \iint_{\Omega_{k-N}} u_{k} \lesssim 2^{-k \alpha}\left(2^{k} r\right)^{-n-2} \sum_{I \in \mathcal{W}_{k}} \iint_{I} u_{k}
$$

Note that for each $I \in \mathcal{W}_{k}$, by the definition of $I^{*}$, we may fix a point $\left(Y_{I}, s_{I}\right) \in \partial I^{*}$ such that $s_{I}>T_{I}+v \ell(I)^{2}$. Note also that in particular, $\left(Y_{I}, s_{I}\right) \in \Omega_{k-3}$, by (3.2.14).

For every $(Y, s) \in I$, by (3.2.11) we then have that by Harnack's inequality,

$$
u_{k}(Y, s)=\iint_{\mathcal{R}_{k} \cap\left\{T_{0}<\tau\right\}} f_{k}(z, \tau) d \omega^{Y, s}(z, \tau) \lesssim \iint_{\mathcal{R}_{k} \cap\left\{T_{0}<\tau\right\}} f_{k}(z, \tau) d \omega^{Y_{I}, S_{I}}(z, \tau)
$$

Recall that since we are treating the case $T_{\min }>-\infty, R_{0}<\infty$, we need only consider $k$ such that

$$
2^{k} r \leq R_{0} \leq \sqrt{\left(T_{0}-T_{\min }\right) /\left(100 \kappa_{0}\right)}
$$

We now choose a collection of surface cubes $\mathcal{F}_{k}=\left\{\Delta_{i}^{k}=Q_{i}^{k} \cap \Sigma\right\}_{i}$, of parabolic sidelength $\ell\left(Q_{i}^{k}\right)=\sqrt{\kappa_{0}} 2^{k} r / 100$, whose union covers $\mathcal{R}_{k} \cap\left\{T_{0}<\tau\right\}$. Note that we may do this in such a way that each $\Delta_{i}^{k} \subset \Delta_{k+3} \backslash \Delta_{k-1}$, and the cardinality of $\mathcal{F}_{k}$ is at most $C\left(n, \kappa_{0}\right)$, uniformly in $k$. Note further that by construction, the reverse Hölder estimate (3.2.2) may be applied uniformly in each $\Delta_{i}^{k} \in \mathcal{F}_{k}$, with pole at $\left(Y_{I}, s_{I}\right)$. Consequently, for each $I \in \mathcal{W}_{k}$,

$$
\iint_{I} u_{k} \lesssim|I| \sum_{\mathcal{F}_{k}} \iint_{\Delta_{i}^{k}} f_{k}(z, \tau) d \omega^{Y_{I}, s_{I}}(z, \tau)
$$




$$
\begin{aligned}
\leq|I| \sum_{\mathcal{F}_{k}} \sigma\left(\Delta_{i}^{k}\right)\left(f f_{\Delta_{i}^{k}} f^{p} d \sigma\right)^{1 / p}\left(f f_{\Delta_{i}^{k}}\left(k^{Y_{I}, s_{I}}\right)^{q} d \sigma\right)^{1 / q} \\
\quad \lesssim|I| \sum_{\mathcal{F}_{k}}\left(f_{\Delta_{i}^{k}} f^{p} d \sigma\right)^{1 / p} \lesssim_{\kappa_{0}}|I|\left(\mathcal{M}\left(f^{p}\right)(x, t)\right)^{1 / p},
\end{aligned}
$$

where in the last step we have used the bound on $\operatorname{card}\left(\mathcal{F}_{k}\right)$, along with ADR and the fact that $\operatorname{diam}\left(\Delta_{i}^{k}\right) \approx_{\kappa_{0}} \operatorname{diam}\left(\Delta_{k+3}\right)$. Note that (3.2.14) implies in particular that $\sum_{I \in \mathcal{W}_{k}}|I| \lesssim\left(2^{k} r\right)^{n+2}$. Plugging estimate (3.2.16) into (3.2.15), we therefore obtain the claimed bound (3.2.13), and hence that (1) implies (2).

Remark 3.2.17. It is well known that if $k^{Y, s}$ satisfies the reverse Hölder estimate (3.2.2) for $q>1$, then $k^{Y, s}$ satisfies (3.2.2) for some $\tilde{q}>q$. Therefore, not only do we obtain (3.2.3) for $1<p<\infty$ where $1 / p+1 / q=1$, but for $\tilde{p}<p$ where $1 / \tilde{p}+1 / \tilde{q}=1$.

(2) implies (3). It is enough to show

$$
\left\|N_{*} u\right\|_{L^{p}(\Sigma)} \lesssim_{\kappa_{1}}\|f\|_{L^{p}(\Sigma)}
$$

for $f \in C_{c}(\Sigma)$ as $C_{c}(\Sigma)$ is a dense subset of $L^{p}(\Sigma)$. Let $\kappa_{1} \in(0,1), T_{0}-T_{\min } \geq \kappa_{1} R_{0}^{2}$, and $f \in C_{c}(\Sigma)$ with compact support in $\Sigma^{T_{0}}$. By assumption and Remark 3.2.17 we have for all $(x, t) \in \Sigma^{T_{0}}$

$$
N_{*} u(x, t) \lesssim_{K_{1}}\left(\mathcal{M}\left(|f|^{\tilde{p}}\right)(x, t)\right)^{1 / \tilde{p}}
$$

for some $\tilde{p}<p$ where $1 / \tilde{p}+1 / \tilde{q}=1$. As $p / \tilde{p}>1$, by the boundedness of $\mathcal{M}$ on $L^{p / \tilde{p}}(\Sigma)$ and (3.2.18) we then obtain

$$
\iint_{\Sigma}\left(N_{*} u\right)^{p} d \sigma \lesssim_{\kappa_{1}} \iint_{\Sigma}\left(\mathcal{M}\left(|f|^{\tilde{p}}\right)\right)^{p / \tilde{p}} d \sigma \lesssim_{\kappa_{1}} \iint_{\Sigma}|f|^{p} d \sigma
$$

proving (2) implies (3). 
Remark 3.2.19. In order for the non-tangential convergence to hold in a non-vacuous way, one should impose some extra assumption to guarantee that the "cones" defined in (3.2.6) above are non-empty at infinitely many scales less than one, $\sigma$ almost everywhere on $\Sigma$; an interior corkscrew condition is more than enough.

(3) implies (1). Fix $\kappa_{0} \in(0,1)$, and a point $\left(x_{0}, t_{0}\right) \in \Sigma$, with $t_{0}-T_{\min } \geq \kappa_{0} R_{0}^{2}$. Let $0<r<\sqrt{\kappa_{0}} R_{0} / 2$, and set $\Delta=Q_{r}\left(x_{0}, t_{0}\right) \cap \Sigma$. Our goal is to show that the reverse Hölder estimate (3.2.2) holds for this $\Delta$, and to this end, it is actually enough to verify (3.2.2) uniformly for each $\Delta^{\prime}=Q_{\varepsilon r}\left(x_{1}, t_{1}\right) \cap \Sigma \subset \Delta$, with $\left(x_{1}, t_{1}\right) \in \Delta$, where $\varepsilon$ is a fixed small positive number to be chosen. Indeed, the reverse Hölder estimate for all such $\Delta^{\prime}$, with pole $(Y, s) \in \Omega \backslash Q_{4 r}\left(x_{0}, t_{0}\right)$, implies that for $\Delta$, with constants depending on $\varepsilon$. In turn, by Lemma 3.1.5 and Lemma 3.1.24, it is enough to show that there are uniform constants $\theta, \beta \in(0,1)$ such that for every $(X, t) \in Q_{2 \varepsilon r}\left(x_{1}, t_{1}\right) \cap \Omega$, if $E \subset \Delta_{X, t}$ is a Borel set,

$$
\sigma(E) \geq(1-\theta) \sigma\left(\Delta_{X, t}\right) \Longrightarrow \omega^{X, t}(E) \geq \beta
$$

where as above, $\Delta_{X, t}:=\Sigma \cap Q_{X, t}:=\Sigma \cap Q\left((X, t), 20 \frac{M_{1}}{a} \delta(X, t)\right)$.

We fix $\Delta^{\prime}$ and $(X, t)$ as above. Let $(\hat{x}, \hat{t}) \in \Sigma$ be a touching point for $(X, t)$ with respect to the usual parabolic metric; i.e., $\delta(X, t)=\|(X, t)-(\hat{x}, \hat{t})\|$. We now choose $\varepsilon$ small enough, depending on $M_{1}, a$, and $\kappa_{0}$, such that for $(X, t) \in Q_{2 \varepsilon r}\left(x_{1}, t_{1}\right) \cap \Omega$, where $\left(x_{1}, t_{1}\right) \in \Delta$ is the center of $\Delta^{\prime}$, we have $200 M_{1} \delta(X, t) / a<\sqrt{\kappa_{0}} R_{0} / 1000$, and also

$$
\min \left(t-T_{\min }, \hat{t}-T_{\min }, T_{\min }\left(\Delta^{\prime}\right)-T_{\min }, T_{\min }\left(\Delta_{X, t}\right)-T_{\min }\right)>\kappa_{0} R_{0}^{2} / 2
$$

Set $\kappa_{1}=\kappa_{0} / 100$, and set $T_{0}:=T_{\min }+\kappa_{1} R_{0}^{2}$. Let $f \in C_{c}\left(\Delta_{X, t}\right)$ be non-negative, with $\|f\|_{L^{p}(\Sigma)} \leq 1$. By assumption, the solution $u$ to the initial-Dirichlet problem in $\Omega^{T_{0}}$, with 
data $f$, enjoys the estimate

$$
\left\|N_{*} u\right\|_{L^{p}(\Sigma)} \lesssim_{K_{0}}\|f\|_{L^{p}(\Sigma)} \approx_{\kappa_{0}} 1
$$

for some $p<\infty$.

Set $r^{\prime}:=\delta(X, t) / 10$. Let $I \in \mathcal{W}$ be a Whitney cube containing $(X, t)$, and note that $I \in \mathcal{W}(z, \tau)$, for every $(z, \tau) \in \Delta^{\prime \prime}:=Q_{r^{\prime}}(\hat{x}, \hat{t}) \cap \Sigma($ see $(3.2 .5))$ and therefore

$$
u(Y, s) \lesssim\left(f_{\Delta^{\prime \prime}}\left(N_{*} u\right)^{p} d \sigma\right)^{1 / p}, \quad \forall(Y, s) \in I^{*}
$$

(see (3.2.6)-(3.2.7)). Thus, by [M, Theorem 3], we have

$$
u(X, t) \lesssim\left(f_{I^{*}}(u(Y, s))^{p} d Y d s\right)^{1 / p} \lesssim\left(f_{\Delta^{\prime \prime}}\left(N_{*} u\right)^{p} d \sigma\right)^{1 / p} \lesssim \delta(X, t)^{-(n+1) / p}
$$

where in the last step we have applied the lower ADR estimate to $\Delta^{\prime \prime}$, and used (3.2.21). In turn, taking a supremum over all non-negative $f \in C_{c}\left(\Delta_{X, t}\right)$ such that $\|f\|_{p} \leq 1$, we obtain by Riesz representation that

$$
\left(\iint_{\Delta_{X, t}}\left(k^{X, t}\right)^{q} d \sigma\right)^{1 / q} \lesssim \delta(X, t)^{-(n+1) / p}
$$

with $q=p /(p-1)$.

We now claim that the latter estimate implies (3.2.20), for suitable $\theta, \beta \in(0,1)$, in which case we are done. To prove this claim, note first that by ADR,

$$
\sigma\left(\Delta_{X, t}\right) \approx \delta(X, t)^{n+1}
$$

Let $E \subset \Delta_{X, t}$ satisfy the left hand estimate in (3.2.20), and set $A:=\Delta_{X, t} \backslash E$, for $\theta>0$ to be chosen, so that

$$
\sigma(A) \leq \theta \sigma\left(\Delta_{X, t}\right)
$$

Then 


$$
\begin{aligned}
\omega^{X, t}(A) \leq \sigma(A)^{1 / p}\left(\iint_{\Delta_{X, t}}\left(k^{X, t}\right)^{q} d \sigma\right)^{1 / q} & \\
& \lesssim \sigma(A)^{1 / p} \delta(X, t)^{-(n+1) / p} \lesssim \theta^{1 / p} \approx \theta^{1 / p} \omega^{X, t}\left(\Delta_{X, t}\right),
\end{aligned}
$$

where in the last three steps we have used (3.2.22)-(3.2.24), and then Lemma 2.0.5. Taking complements, and using Lemma 2.0.5 once again, for $\theta$ small enough we obtain (3.2.20).

\subsection{BMO Solvability}

\subsubsection{Introduction}

In the setting of divergence form elliptic PDE, it is well known that solvability of the Dirichlet problem with $L^{p}$ data is equivalent to scale-invariant absolute continuity of ellipticharmonic measure (specifically that elliptic-harmonic measure belongs to the Muckenhoupt weight class $A_{\infty}$ with respect to surface measure on the boundary). To be more precise, in a Lipschitz or even chord-arc domain, one obtains that the Dirichlet problem is solvable with data in $L^{p}(\Omega)$ for some $1<p<\infty$, if and only if elliptic-harmonic measure $\omega$ with some fixed pole is absolutely continuous with respect to surface measure $\sigma$ on the boundary, and the Poisson kernel $d \omega / d \sigma$ satisfies a reverse Hölder condition with exponent $p^{\prime}=p /(p-1)$; see the monograph of Kenig [Ke], and the references cited there. In fact, the equivalence between $L^{p}$ solvability and quantitative absolute continuity holds much more generally, for any open set with an Ahlfors-David regular boundary (see [HLe] for a proof, although the result is somewhat folkloric); in this generality, the $A_{\infty} /$ reverse-Hölder property is (necessarily) replaced by its weak version, which does not entail doubling.

These results have endpoint versions, as well: in [DKP], Dindos, Kenig and Pipher showed that in a Lipschitz domain (or even a chord-arc domain) elliptic-harmonic measure satisfies an $A_{\infty}$ condition with respect to surface measure, if and only if a natural Carleson 
measure/BMO estimate holds for solutions of the Dirichlet problem with continuous data. The results of $[\mathrm{DKP}]$ have been extended to the setting of a 1-sided Chord-arc domain by Z. Zhao [Z].

In the above works, the proofs relied substantially on quantitative connectivity of the domain, in the form of the Harnack Chain condition. More recently, Hofmann and P. Le [HLe] proved an analogous result in the absence of any connectivity hypothesis, either quantitative or qualitative: one obtains that BMO solvability implies scale invariant quantitative absolute continuity (the weak- $A_{\infty}$ property) of elliptic-harmonic measure with respect to surface measure on $\partial \Omega$, assuming only that $\Omega$ is an open set with Ahlfors-David regular boundary.

The goal of the present section is to extend the results of [HLe] to the parabolic setting. As regards geometric hypotheses, we assume only that $\Omega \subset \mathbb{R}^{n+1}$ is an open set whose boundary satisfies an appropriate version of a parabolic Ahlfors-David regularity condition. In particular, we impose no connectivity hypothesis, such as a parabolic Harnack chain condition.

\subsubsection{Main Result}

Given an open set $\Omega \subset \mathbb{R}^{n+1}$ and a divergence form parabolic operator $L$ as above, for which the continuous Dirichlet problem is solvable, we shall say that the initial-Dirichlet problem (see Definition 1.2.13) is BMO-solvable for $L$ in $\Omega$ if for all continuous $f$ with compact support on $\Sigma$, the solution $u$ of the initial-Dirichlet problem with data $f$ satisfies the Carleson measure estimate

$$
\begin{aligned}
\sup _{(x, t) \in \Sigma, 0<r<R(t)} \frac{1}{\sigma\left(\Delta_{r}\right)} \iint_{\Omega \cap Q_{r}(x, t)}\left(|\nabla u(Y, s)|^{2}+\left|\delta(Y, s) \partial_{s} u(Y, s)\right|^{2}\right) \delta(Y, s) d Y d s & \leq C\|f\|_{B M O(\Sigma)}^{2}
\end{aligned}
$$


where $\Delta_{r}:=Q_{r}(x, t) \cap \Sigma$, and $R(t):=\min \left(R_{0}, \sqrt{t-T_{\min }} /(4 \sqrt{n})\right)$, with $R_{0}:=\operatorname{diam}(\Sigma)$. We recall that $T_{\max }-T_{\min } \gtrsim R_{0}^{2}$; see the discussion preceeding Theorem 3.2.1.

Theorem 3.3.2. Let $L$ be a divergence form parabolic operator defined on $\Omega$. Let $\Sigma$ be globally time-backwards $A D R$, and assume further that if $R_{0}:=\operatorname{diam} \Sigma=\infty$, then $T_{\min }=$ $-\infty$.

If the initial-Dirichlet problem for $L$ is BMO-solvable in $\Omega$, then the parabolic measure belongs to weak- $A_{\infty}$ in the following sense: for every parabolic cube $Q:=Q_{r}\left(x_{0}, t_{0}\right)$, with $\left(x_{0}, t_{0}\right) \in \Sigma$ and $0<r<\min \left(R_{0}, \sqrt{t_{0}-T_{\min }} /(4 \sqrt{n})\right)$, and for all $(Y, s) \in \Omega \backslash 4 Q$, parabolic measure $\omega_{L}^{Y, s} \in$ weak- $A_{\infty}(\Delta)$, where the parameters in the weak- $A_{\infty}$ condition are uniform in $\Delta$.

We note that we are implicitly assuming here, as above, that the continuous Dirichlet problem is solvable for $L$; we know that this is true if $L$ is the heat operator, or if the coefficients of $L$ are $C^{1}$-Dini: see Remarks 1.2.21 and 1.2.23. We note that our assumption of solvability of the continuous Dirichlet problem is used only qualitatively: the constants in our estimates will depend only on dimension, ellipticity, and the constant in the BMOsolvability estimate (3.3.1).

Remark 3.3.3. By Theorem 3.2.1, the weak- $A_{\infty}$ property of caloric (or parabolic) measure is equivalent to $L^{p}$ solvability of the initial-Dirichlet problem, for some $p<\infty$; hence the latter also follows from BMO solvability.

Remark 3.3.4. In the elliptic case, the analogue of Theorem 3.3.2 has a partial converse [HLe, Theorem 1.6], valid for the Laplacian: under the additional assumption that $\Omega$ satisfies an interior Corkscrew condition, if $\partial \Omega$ is $\mathrm{ADR}$, and harmonic measure belongs to weak- $A_{\infty}$ with respect to surface measure on $\partial \Omega$, then the Dirichlet problem for Laplace's 
equation is BMO-solvable. In the parabolic setting, this converse result remains open. The proof in the elliptic case relies on square-function/non-tangential-maximal-function estimates, which in turn are obtained by invoking results of [HM] (see also [HLMN], [MT]) to deduce uniform rectifiability of $\partial \Omega$; see [HM], [HMM1], [HMM2] (as well as [GMT], [AGMT] for related converse results). The machinery created in these references, and exploited in [HLe], has yet to be developed in the parabolic setting.

Proof of Theorem 3.3.2. Recall that for $(X, t) \in \Omega$, we let $\delta_{\infty}(X, t):=\operatorname{dist}_{\infty}\left((X, t), \partial_{e} \Omega\right)$ denote the parabolic $\ell^{\infty}$ distance to the essential boundary, and that if $\sqrt{t-T_{\min }}>\delta_{\infty}(X, t)$, then

$$
\delta_{\infty}(X, t)=\operatorname{dist}_{\infty}((X, t), \Sigma),
$$

by definition of $\Sigma$. We note that in the context of Theorem 3.3.2, by hypothesis we shall always work with points $(X, t)$ for which (3.3.5) holds.

Throughout the present section, "touching points" will always be taken with respect to the $\ell^{\infty}$ parabolic distance (see Definition 1.2.11).

Given $(X, t) \in \Omega$, let $(\hat{x}, \hat{t}) \in \Sigma$ be a touching point for $(X, t)$, so that

$$
r:=\delta_{\infty}(X, t)=\|(X, t)-(\hat{x}, \hat{t})\|_{\ell^{\infty}},
$$

and define $\Delta_{X, t}$ as in (2.0.17) where $M_{2}$ is the constant in Remark 2.0.16:

$$
\Delta_{X, t}:=\Sigma \cap Q\left((\hat{x}, \hat{t}), M_{2} r\right) .
$$

Recall that caloric (or parabolic) measure $\omega^{X, t}$ is locally ample on $\Delta_{X, t}$, or more precisely, $(\theta, \beta)$-locally ample, if there exists constants $\theta, \beta \in(0,1)$ such that

$$
\sigma(F) \geq(1-\theta) \sigma\left(\Delta_{X, t}\right) \quad \Longrightarrow \quad \omega^{X, t}(F)=\omega_{L}^{X, t}(F) \geq \beta,
$$


where $F \subset \Delta_{X, t}$ is a Borel set.

To prove Theorem 3.3.2, we suppose that $\Sigma$ is globally ADR and TBADR, and observe that it suffices to verify the hypotheses of Theorem 3.1.3, in the presence of BMOsolvability. More precisely, we suppose that estimate (3.3.1) holds for all $f \in C_{c}\left(\Sigma \cap\left\{T_{\min }<\right.\right.$ $\left.\left.t<T_{\max }\right\}\right)$, and our goal is to verify the $(\theta, \beta)$-locally ampleness condition (3.3.7), for all $(X, t) \in \Omega$ with $\left.2 M_{2} \delta_{\infty}(X, t)<\min \left(R_{0}, \sqrt{t-T_{\min }}\right)\right)$, where $M_{2}$ is the constant in Remark 2.0.16. In comparing this constraint on $\delta_{\infty}(X, t)$ with that on $r$ in Theorem 3.1.3, we observe that there is no loss of generality: indeed, for a fixed large constant $M$, we may cover a given surface cube $\Delta_{r}\left(x_{0}, t_{0}\right)$ by surface cubes of scale $r / M$; it then suffices to verify the Reverse Hölder inequality (3.1.4) on these smaller cubes.

Remark 3.3.8. In the proof of Theorem 3.1.3, $\Delta_{X, t}$ is defined in a slightly different way: there, $\Delta_{X, t}$ is centered at $(X, t) \in \Omega$; more precisely, it is of the form $\Sigma \cap Q((X, t), K \delta(X, t))$, for some $K \geq 2$. This is comparable to the present definition of $\Delta_{X, t}$ in Remark 2.0.16.

Fix $(X, t) \in \Omega$, and let $(\hat{x}, \hat{t}) \in \Sigma$ be a touching point for $(X, t)$, so that (3.3.6) holds. Fix a sufficiently small number $b \in(0, \pi / 10,000)$, to be chosen depending only on $n$ and ADR. We then set

$$
\begin{aligned}
Q_{X, t} & :=Q\left((\hat{x}, \hat{t}), M_{2} r\right), \quad \Delta_{X, t}:=\Delta\left((\hat{x}, \hat{t}), M_{2} r\right), \\
Q_{X, t}^{\prime} & :=Q((\hat{x}, \hat{t}), b r), \quad \Delta_{X, t}^{\prime}:=\Delta((\hat{x}, \hat{t}), b r) .
\end{aligned}
$$

Note that $\Delta_{X, t}$ is the same as in (2.0.17).

The proof will use the following pair of claims. We recall that $a$ is the constant in Remark 1.2.25.

Claim 1: For $b$ small enough, depending on $n, a$ and $\mathrm{ADR}$, there is a constant $\beta>0$ depending only on $n, a, b, \mathrm{ADR}$ and $\lambda$, and a cube $Q_{1}:=Q\left(\left(x_{1}, t_{1}\right), b r\right) \subset Q_{X, t}$, with $\left(x_{1}, t_{1}\right) \in$ 
$\Sigma$, such that

$$
\operatorname{dist}\left(Q_{X, t}^{\prime}, Q_{1}\right) \gtrsim_{a} r
$$

(note that the implicit constants in (3.3.9) depend on the constant $a$ in Remark 1.2.21, but not on $b$ ), and

$$
\omega^{X, t}\left(\Delta_{1}\right) \geq \beta \omega^{X, t}\left(\Delta_{X, t}\right)
$$

where $\Delta_{1}:=Q_{1} \cap \Sigma$.

Remark 3.3.11. Since the constant $a$ in Remark 1.2.25 depends only on $n$ and ADR, in turn $b$ ultimately depends only on $n$ and ADR.

Claim 2: Suppose that $u$ is a non-negative solution of $L u=0$ in $\Omega$, vanishing continuously on $2 \Delta_{X, t}^{\prime}$, with $\|u\|_{L^{\infty}(\Omega)} \leq 1$. Then for every $\epsilon>0$,

$$
u^{2}(X, t) \leq \frac{C_{\epsilon}}{\sigma\left(\Delta_{X, t}\right)} \iint_{Q_{X, t} \cap \Omega}\left(|\nabla u(Y, s)|^{2}+\left|\delta(Y, s) \partial_{s} u(Y, s)\right|^{2}\right) \delta(Y, s) d Y d s+C \epsilon^{2 \alpha}
$$

where $\alpha$ is the exponent from Lemma 2.0.21.

Momentarily taking these two claims for granted, we adapt to the parabolic setting the argument of [DKP], as modified in [HLe]. Let $Q_{1}$ and $\Delta_{1}$ be as in Claim 1. Let $F \subset \Delta_{X, t}$ be a Borel set satisfying

$$
\sigma(F) \geq(1-\eta) \sigma\left(\Delta_{X, t}\right)
$$

for some small $\eta>0$. If we choose $\eta$ small enough, depending only on $n$, ADR, and $a$, then by inner regularity of $\sigma$, there is a closed set $F_{1} \subset F \cap \Delta_{1}$ such that

$$
\sigma\left(F_{1}\right) \geq(1-\sqrt{\eta}) \sigma\left(\Delta_{1}\right)
$$

Set $A_{1}:=\Delta_{1} \backslash F_{1}$. Then $A_{1}$ is relatively open in $\Sigma$. Define

$$
f:=\max \left(0,1+\gamma \log \mathcal{M}\left(1_{A_{1}}\right)\right),
$$


where $\gamma>0$ is a small number, to be chosen, and $\mathcal{M}$ is the Hardy-Littlewood maximal operator on $\Sigma$. Note that we have the following:

$$
0 \leq f \leq 1, \quad\|f\|_{B M O(\Sigma)} \leq C \gamma, \quad 1_{A_{1}}(\mathbf{x}) \leq f(\mathbf{x}), \forall \mathbf{x} \in \Sigma
$$

Note also that if $\mathbf{z} \in \Sigma \backslash 2 Q_{1}$, then

$$
\mathcal{M}\left(1_{A_{1}}\right)(\mathbf{z}) \lesssim \frac{\sigma\left(A_{1}\right)}{\sigma\left(\Delta_{1}\right)} \lesssim \sqrt{\eta}
$$

where the implicit constants depend only on $n$ and ADR. Thus, if $\eta$ is chosen small enough depending on $\gamma$, then $1+\gamma \log \mathcal{M}\left(1_{A_{1}}\right)$ will be negative, hence $f \equiv 0$, on $\Sigma \backslash 2 Q_{1}$.

In order to work with continuous data, we shall require the following.

Lemma 3.3.14. There exists a collection of continuous functions $\left\{f_{v}\right\}_{0<v<a r / 1000}$, defined on $\Sigma$ with the following properties.

1. $0 \leq f_{v} \leq 1$, for each $v$.

2. $\operatorname{supp}\left(f_{v}\right) \subset 3 Q_{1} \cap \Sigma$.

3. $1_{A_{1}}(\mathbf{x}) \leq \liminf _{v \rightarrow 0} f_{v}(\mathbf{x})$, for every $\mathbf{x} \in \Sigma$.

4. $\sup \left\|f_{v}\right\|_{B M O(\Sigma)} \leq C\|f\|_{B M O(\Sigma)} \lesssim \gamma$, where $C=C(n, A D R)$.

We defer the proof of Lemma 3.3.14 to the end of this section.

Taking the two claims (and Lemma 3.3.14) for granted momentarily, we give the proof of Theorem 3.3.2. As noted above, by Theorem 3.1.3, it suffices to verify the $(\theta, \beta)$-locally ampleness condition (3.3.7). To this end, let $u_{v}$ be the solution of the continuous Dirichlet problem with data $f_{v}$. Then $f_{v}$ vanishes on $2 \Delta_{X, t}^{\prime}$, by the separation condition (3.3.9) in Claim 1 and Lemma 3.3.14-(2), provided that $b$ is chosen small enough depending on $a$. 
Then, for small $\epsilon>0$ to be chosen momentarily, by Lemma 3.3.14, Fatou's lemma, and Claim 2, we have

$$
\omega^{X, t}\left(A_{1}\right) \leq \int_{\Sigma} \liminf _{v \rightarrow 0} f_{v} d \omega^{X, t} \leq \liminf _{\nu \rightarrow 0} u_{v}(X, t) \leq C_{\epsilon} \gamma+C \epsilon^{\alpha}
$$

where in the last inequality we used (3.3.12), (3.3.1), and Lemma 3.3.14-(4). Combining (3.3.15) with (2.0.18), we find that

$$
\omega^{X, t}\left(A_{1}\right) \leq\left(C_{\epsilon} \gamma+C \epsilon^{\alpha}\right) \omega^{X, t}\left(\Delta_{X, t}\right)
$$

Next, we set $A:=\Delta_{X, t} \backslash F$, and observe that by definition of $A$ and $A_{1}$, along with Claim 1, and (3.3.16),

$$
\omega^{X, t}(A) \leq \omega^{X, t}\left(\Delta_{X, t} \backslash \Delta_{1}\right)+\omega^{X, t}\left(A_{1}\right) \leq\left(1-\beta+C_{\epsilon} \gamma+C \epsilon^{\alpha}\right) \omega^{X, t}\left(\Delta_{X, t}\right)
$$

We now choose first $\epsilon>0$, and then $\gamma>0$, so that $C_{\epsilon} \gamma+C \epsilon^{\alpha}<\beta / 2$, to obtain that

$$
\omega^{X, t}(F) \geq \frac{\beta}{2} \omega^{X, t}\left(\Delta_{X, t}\right) \geq c \beta
$$

where in the last inequality we have used (2.0.18). Therefore (3.3.7) holds.

It remains to prove the two claims. Let $a>0$ be the constant mentioned in Remark 1.2.25. Recall that $M_{1}$ is the constant in Lemma 2.0.5, and that $M_{2}$ is the constant in Remark 2.0.16.

Proof of Claim 1. Recall that we have fixed $(X, t) \in \Omega$, and that $(\hat{x}, \hat{t}) \in \Sigma$ is a touching point for $(X, t)$, so that $(\hat{x}, \hat{t})$ lies on the boundary of the (open) cube $Q_{r}(X, t)$, with $r=$ $\delta_{\infty}(X, t)=\|(X, t)-(\hat{x}, \hat{t})\|_{\ell^{\infty}}$, and $Q_{r}(X, t) \cap \Sigma=\emptyset$. If there is more than one touching point, we simply fix one. Note that since $(\hat{x}, \hat{t}) \in \partial Q_{r}(X, t)$, we have in particular that

$$
\hat{t} \leq t+r^{2}
$$


Consequently, we may apply Remark 1.2 .25 to the cube $Q_{b i g}:=Q_{2 a^{-1} r}(\hat{x}, \hat{t})$, to find a point $(y, s) \in \Sigma \cap Q_{b i g}$, with $s<\hat{t}-(2 r)^{2} \leq t+r^{2}-(2 r)^{2}$. The point $(y, s)$ therefore satisfies

$$
s<t-3 r^{2}, \text { and }\|(X, t)-(y, s)\| \lesssim_{a} r .
$$

Let us note for future reference that for $(Z, \tau) \in \Omega \cap Q_{b i g}$, by Remark 1.2.23 we have

$$
\operatorname{dist}_{\infty}((Z, \tau), \Sigma)=\delta_{\infty}(Z, \tau) \approx_{a} \operatorname{dist}_{\infty}((Z, \tau), \partial \Omega), \text { if } \tau<t-(r / 4)^{2},
$$

since $(X, t) \in \Omega$ implies that $t<T_{\max }$, and the restriction $\sqrt{t-T_{\min }}>2 M_{2} r$, with $M_{2} \approx$ $M_{1} / a \gg 1 / a$, implies that (3.3.5) holds for $(Z, \tau) \in Q_{b i g}$.

We fix a point $\mathbf{X}_{*}=\left(X_{*}, t_{*}\right)$ lying on the back face of $Q_{r}(X, t)$ (so that $\left.t_{*}=t-r^{2}\right)$, with

$$
\left|X_{*}-\hat{x}\right| \geq r / 4 .
$$

We now form the parabola $\mathcal{P}_{1}$ with vertex at $(y, s)$, passing through the point $\left(X_{*}, t_{*}\right)$, so that any point $(Z, \tau)$ on $\mathcal{P}_{1}$ satisfies

$$
\tau-s=\frac{t_{*}-s}{\left|X_{*}-y\right|^{2}}|Z-y|^{2} \gtrsim_{a}|Z-y|^{2} .
$$

We also form the parabola $\mathcal{P}_{2}$, with vertex at $\left(X_{*}, t_{*}\right)$, through the point $(X, t)$, so that any point $(Z, \tau)$ on $\mathcal{P}_{2}$ satisfies

$$
\tau-t_{*}=\frac{t-t_{*}}{\left|X-X_{*}\right|^{2}}\left|Z-X_{*}\right|^{2} \gtrsim\left|Z-X_{*}\right|^{2}
$$

(it may be that $X_{*}=X$, in which case $\mathcal{P}_{2}$ is simply the horizontal line joining $\left(X, t-r^{2}\right)$ to $(X, t))$. Set $C:=\mathcal{P}_{1} \cup \mathcal{P}_{2}$, and travel along $C$ backwards in time, starting at $(X, t)$, moving towards $\left(X_{*}, t_{*}\right)$, and if need be through $\left(X_{*}, t_{*}\right)$ towards $(y, s)$, stopping the first time that we reach a point $\left(Z_{1}, \tau_{1}\right)$ satisfying

$$
\delta_{\infty}\left(Z_{1}, \tau_{1}\right)=b M_{2}^{-1} r .
$$


Choose $\left(x_{1}, t_{1}\right) \in \Sigma$ such that $\delta_{\infty}\left(Z_{1}, \tau_{1}\right)=\left\|\left(Z_{1}, \tau_{1}\right)-\left(x_{1}, t_{1}\right)\right\|_{\ell^{\infty}}$, set $\Delta_{1}:=Q_{1} \cap \Sigma$, with $Q_{1}:=Q\left(\left(x_{1}, t_{1}\right), b r\right)$, so that, by Remark 2.0.16,

$$
\omega^{Z_{1}, \tau_{1}}\left(\Delta_{1}\right) \geq \kappa
$$

We may then move along $C$, forwards in time, from $\left(Z_{1}, \tau_{1}\right)$ to $(X, t)$, to obtain $(3.3 .10)$ by Harnack's inequality and (3.3.18), and the fact that $\omega^{X, t}$ is a probability measure.

Moreover, by (3.3.19) and the construction of the curve $C$, for $b$ small enough depending on $a$, we readily obtain the separation condition (3.3.9), and for $M_{2}$ large enough, again depending on $a$, using the second inequality in (3.3.17), we obtain the containment $Q_{1} \subset$ $Q_{X, t}$

Proof of Claim 2. By a translation, we may suppose that the touching point $(\hat{x}, \hat{t})$ is the origin. As above, we set

$$
r:=\delta_{\infty}(X, t)=\|(X, t)\|_{\ell^{\infty}}
$$

where we have used that $(\hat{x}, \hat{t})=0$. Since the $\ell^{2}$ and $\ell^{\infty}$ versions of the parabolic distance are comparable, we have that

$$
r_{1}:=\|(X, t)\| \approx \delta(X, t) \approx r
$$

with implicit constants depending only on dimension.

Set

$$
P_{X, t}:=Q_{c r}(X, t), \quad P_{X, t}^{-}:=Q_{c r}^{-}(X, t),
$$

where $c<1 / 1000$ is a small fixed positive constant to be chosen momentarily. Then by [M, Theorem 3], we have that

$$
u(X, t) \lesssim\left(\oiint_{P_{X, t}^{-}}|u(\mathbf{Y})|^{2} d \mathbf{Y}\right)^{1 / 2}
$$


Let $S_{0}$ denote the spherical cap

$$
S_{0}:=\left\{(\zeta, \tau) \in \mathbb{S}^{n}:\left\|(\zeta, \tau)-\pi_{\mathrm{p} a r}(X, t)\right\|<\pi / 1000\right\}
$$

For the sake of notational convenience, we shall write

$$
\xi=(\zeta, \tau), \quad \rho^{(1,2)} \xi:=\left(\rho \zeta, \rho^{2} \tau\right)
$$

to denote, respectively, points on the unit sphere $\mathbb{S}^{n}$, and on the parabolic sphere of radius $\rho$ (expressed in parabolic polar coordinates; see Definition 1.2.31).

Then for $c$ in (3.3.21) chosen small enough, we have that

$$
P_{X, t}^{-} \subset \mathcal{A}_{X, t}
$$

where $\mathcal{A}_{X, t}$ is the region given in parabolic polar coordinates by

$$
\mathcal{A}_{X, t}:=\left\{\rho^{(1,2)} \xi: \xi \in S_{0}, r_{1} / 2<\rho<R(\xi)\right\}
$$

where $r_{1} \approx r$ is defined in (3.3.20), and $R(\xi)$ is defined appropriately so that $R(\xi) \lesssim r$, uniformly in $\xi$, and so that $\mathcal{A}_{X, t} \subset \Omega$. In fact, more generally,

$$
\Gamma_{X, t}:=\left\{\rho^{(1,2)} \xi: \xi \in S_{0}, 0<\rho<R(\xi)\right\} \subset \Omega .
$$

Of course, $\Gamma_{X, t}$ is just a truncated version of the parabolic cone $\Gamma$ (see Definition 1.2.33) with vertex at $0=(\hat{x}, \hat{t})$, in the direction $\pi_{\mathrm{p} a r}(X, t)$, with aperture $\pi / 1000$.

Then by (3.3.22) and the fact that $P_{X, t}^{-} \subset \mathcal{A}_{X, t}$, we have

$$
\begin{aligned}
u(X, t) & \lesssim\left(r^{-n-2} \int_{S_{0}} \int_{r_{1} / 2}^{R(\xi)}\left|u\left(\rho^{(1,2)} \xi\right)\right|^{2} \rho^{n+1} d \rho d \mu(\xi)\right)^{1 / 2} \\
& \lesssim\left(r^{-n-2} \int_{S_{0}} \int_{r_{1} / 2}^{R(\xi)}\left|u\left(\rho^{(1,2)} \xi\right)-u\left((\epsilon r)^{(1,2)} \xi\right)\right|^{2} \rho^{n+1} d \rho d \mu(\xi)\right)^{1 / 2}+O\left(\epsilon^{\alpha}\right) \\
& =: I+O\left(\epsilon^{\alpha}\right)
\end{aligned}
$$


where we have used parabolic polar coordinates (Definition 1.2.31), and where the "big-O" term

$$
\left(r^{-n-2} \int_{S_{0}} \int_{r_{1} / 2}^{R(\xi)}\left|u\left((\epsilon r)^{(1,2)} \xi\right)\right|^{2} \rho^{n+1} d \rho d \mu(\xi)\right)^{1 / 2} \approx\left(\int_{S_{0}}\left|u\left((\epsilon r)^{(1,2)} \xi\right)\right|^{2} d \mu(\xi)\right)^{1 / 2}
$$

has been estimated using first that $r_{1} \approx r \approx R(\xi)$, and then Lemma 2.0.21 and the fact that $u$ vanishes continuously on $2 \Delta_{X, t}^{\prime}$, which is centered at $(\hat{x}, \hat{t})=0$, and has parabolic diameter $\approx r$.

It remains to control term $I$ by appropriate localized square functions. To this end, using that $\rho \approx r$ in $\mathcal{A}_{X, t}$, we write

$$
\begin{aligned}
I^{2} & =r^{-n-2} \iint_{\mathcal{A}_{X, t}}\left|\int_{\epsilon r}^{\rho} \partial_{q} u\left(q^{(1,2)} \xi\right) d q\right|^{2} \rho^{n+1} d \rho d \mu(\xi) \\
& \lesssim \iint_{\mathcal{A}_{X, t}} \int_{\epsilon r}^{\rho}\left|\nabla u\left(q^{(1,2)} \xi\right)\right|^{2} d q d \rho d \mu(\xi)+\iint_{\mathcal{A}_{X, t}} \int_{\epsilon r}^{\rho} q^{2}\left|\partial_{s} u\left(q^{(1,2)} \xi\right)\right|^{2} d q d \rho d \mu(\xi) \\
& :=I_{1}^{2}+I_{2}^{2}
\end{aligned}
$$

We note first that

$$
\begin{gathered}
I_{1}^{2} \lesssim_{\epsilon} r^{-n-2} \iint_{\mathcal{A}_{X, t}} \int_{\epsilon r}^{\rho}\left|\nabla u\left(q^{(1,2)} \xi\right)\right|^{2} q^{n+2} d q d \rho d \mu(\xi) \\
\lesssim_{\epsilon} r^{-n-1} \int_{S_{0}} \int_{\epsilon r}^{R(\xi)}\left|\nabla u\left(q^{(1,2)} \xi\right)\right|^{2} q^{n+2} d q d \mu(\xi) \\
\approx_{\epsilon} r^{-n-1} \iint_{\mathcal{A}_{*}}|\nabla u(Y, s)|^{2} \delta(Y, s) d Y d s \\
\lesssim_{\epsilon} \sigma\left(\Delta_{X, t}\right)^{-1} \iint_{Q_{X, t} \cap \Omega}|\nabla u(Y, s)|^{2} \delta(Y, s) d Y d s,
\end{gathered}
$$

where the region $\mathcal{A}_{*}$ is given in parabolic polar coordinates by

$$
\mathcal{A}_{*}:=\left\{q^{(1,2)} \xi: \xi \in S_{0}, \epsilon r<q<R(\xi)\right\}
$$

and where in the last step we have used (3.3.20), (3.3.23), ADR, and the definitions of $Q_{X, t}$ and $\Delta_{X, t}$. 
Similarly,

$$
\begin{gathered}
I_{2}^{2} \lesssim_{\epsilon} r^{-n-2} \iint_{\mathcal{A}_{X, t}} \int_{\epsilon r}^{\rho} q^{n+4}\left|\partial_{s} u\left(q^{(1,2)} \xi\right)\right|^{2} d q d \rho d \mu(\xi) \\
\lesssim_{\epsilon} r^{-n-1} \int_{S_{0}} \int_{\epsilon r}^{R(\xi)}\left|\partial_{s} u\left(q^{(1,2)} \xi\right)\right|^{2} q^{n+4} d q d \mu(\xi) \\
\approx_{\epsilon} r^{-n-1} \iint_{\mathcal{A}_{*}}\left|\partial_{s} u(Y, s)\right|^{2} \delta^{3}(Y, s) d Y d s \\
\lesssim_{\epsilon} \sigma\left(\Delta_{X, t}\right)^{-1} \iint_{\mathcal{A}_{*}}\left|\partial_{s} u(Y, s)\right|^{2} \delta^{3}(Y, s) d Y d s .
\end{gathered}
$$

This concludes the proof of Claim 2, and hence of Theorem 3.3.2, modulo the proof of

\section{Lemma 3.3.14.}

Proof of Lemma 3.3.14. Let $\zeta \in C_{0}^{\infty}\left(\mathbb{R}^{n+1}\right)$,

$$
\operatorname{supp}(\zeta) \subset B(0,1), \quad \zeta \equiv 1 \text { on } B(0,1 / 2), \quad 0 \leq \zeta \leq 1
$$

Given $v \in(0, a r / 1000)$, and $\mathbf{x}, \mathbf{z}:=(z, \tau) \in \Sigma$, set

$$
\Lambda_{v}(\mathbf{x}, \mathbf{z}):=b(\mathbf{x}, v)^{-1} \zeta\left(\frac{\mathbf{x}-\mathbf{z}}{v^{\alpha}}\right)
$$

for $\alpha=(1,1, \ldots, 1,2)$, and

$$
b(\mathbf{x}, v):=\iint_{\Sigma} \zeta\left(\frac{\mathbf{x}-\mathbf{z}}{v^{\alpha}}\right) d \sigma(\mathbf{z}) \approx v^{n+1}
$$

uniformly in $\mathbf{x} \in \Sigma$, by the ADR property. Furthermore,

$$
\iint_{\Sigma} \Lambda_{v}(\mathbf{x}, \mathbf{z}) d \sigma(\mathbf{z}) \equiv 1, \quad \forall \mathbf{x} \in \Sigma
$$

We now define

$$
f_{v}(\mathbf{x}):=\iint_{\Sigma} \Lambda_{v}(\mathbf{x}, \mathbf{z}) f(\mathbf{z}) d \sigma(\mathbf{z})
$$


so that $f_{v}$ is continuous, by construction. Let us now verify (1)-(4) of Lemma 3.3.14. We obtain (1) immediately, by (3.3.13), and the properties of $\Lambda_{\nu}$, while (2) follows directly from the smallness of $v$ and the fact that $\operatorname{supp}(f) \subset 2 Q_{1} \cap \Sigma$. Next, observe that since $A_{1}$ is a relatively open set in $\Sigma$, we have that for every $\mathbf{x} \in \Sigma$,

$$
1_{A_{1}}(\mathbf{x}) \leq \liminf _{v \rightarrow 0} \iint_{\Sigma} \Lambda_{v}(\mathbf{x}, \mathbf{z}) 1_{A_{1}}(\mathbf{z}) d \sigma(\mathbf{z}) \leq \liminf _{v \rightarrow 0} f_{v}(\mathbf{x})
$$

by the last inequality in (3.3.13). Hence (3) holds.

To prove (4), we observe that the second inequality is simply a re-statement of the second inequality in (3.3.13), so it suffices to show that

$$
\left\|f_{v}\right\|_{B M O(\Sigma)} \lesssim\|f\|_{B M O(\Sigma)}, \quad \text { uniformly in } v
$$

To this end, we fix a surface cube $\Delta=\Delta(\mathbf{y}, r)$, and we consider two cases.

Case 1: $v \geq r$. In this case, set $c:=f_{\Delta(\mathbf{x}, 2 v)} f$, so that by ADR, (3.3.24) and the construction of $\Lambda_{v}$

$$
f_{\Delta}\left|f_{v}-c\right| d \sigma \lesssim f_{\Delta} f_{\Delta(\mathbf{x}, 2 v)}|f-c| d \sigma d \sigma \lesssim\|f\|_{B M O(\Sigma)}
$$

Case 2: $v<r$. In this case, set $c:=f_{2 \Delta} f$. Then by Fubini's Theorem,

$$
f f_{\Delta}\left|f_{v}(\mathbf{x})-c\right| d \sigma(\mathbf{x}) \lesssim f f_{2 \Delta}|f(\mathbf{z})-c| \iint_{\Sigma} \Lambda_{v}(\mathbf{x}, \mathbf{z}) d \sigma(\mathbf{x}) d \sigma(\mathbf{z}) \lesssim\|f\|_{B M O(\Sigma)}
$$

where again we have used ADR, (3.3.24) and the compact support property of $\Lambda_{v}(\mathbf{x}, \mathbf{z})$.

Since these bounds are uniform all over $\mathbf{y} \in \Sigma$, and $r \in(0$, diam $(\Sigma))$, we obtain (3.3.25). 


\section{Bibliography}

[ABHM] M. Akman, M. Badger, S. Hofmann, J. M. Martell, Rectifiability and elliptic measures on 1-sided NTA domains with Ahlfors-David regular boundaries, Trans. Amer. Math. Soc. 369 (2017), no. 8, 5711-5745.

[AGMT] J. Azzam, J. Garnett, M. Mourgoglou and X. Tolsa, Uniform rectifiability, elliptic measure, square functions, and $\epsilon$-approximability via an ACF monotonicity formula. Preprint 2016. arXiv:1612.02650.

[BL] B. Bennewitz and J.L. Lewis, On weak reverse Hölder inequalities for nondoubling harmonic measures, Complex Var. Theory Appl. 49 (2004), no. 7-9, 571-582.

[BiM] M. Biroli and U. Mosco, Wiener estimates at boundary points for parabolic equations, Annali di Matematica Pura ed Applicata 141, 353367.

[Bo] J. Bourgain, On the Hausdorff dimension of harmonic measure in higher dimension, Invent. Math. 87 (1987), no. 3, 477-483.

[BHL] R. Brown, W. Hu, G. Lieberman, Weak solutions of parabolic equations in noncylindrical domains, Proc. Amer. Math. Soc. 125 (1997), 1785-1792.

[BW] S.-S. Byun and L. Wang, Parabolic equations in time dependent Reifenberg domains, Advances in Mathematics 212 (2007), 797818.

[Ch] M. Christ, A $T(b)$ theorem with remarks on analytic capacity and the Cauchy integral, Colloq. Math., LX/LXI (1990), 601-628.

[CDK] S. Cho, H. Dong and D. Kim, Boundary Value Problems for Parabolic Operators in a Time-Varying Domain, Comm. PDE 40 (2015), 1282-1313.

[Da] B. Dahlberg, On estimates for harmonic measure, Arch. Rat. Mech. Analysis 65 (1977), 272-288.

[DJ] G. David and D. Jerison (1990). Lipschitz approximation to hypersurfaces, harmonic measure, and singular integrals. Indiana Math J., 39, 831-845.

[DKP] M. Dindos, C.E. Kenig, and J. Pipher, BMO solvability and the $A_{\infty}$ condition for elliptic operators, J. Geom. Analysis 21 (2011), 78-95.

[DK] Dong, Hongjie; Kim, Seick Fundamental solutions for second-order parabolic systems with drift terms. Proc. Amer. Math. Soc. 146 (2018), no. 7, 3019-3029.

[D] J. L. Doob, Classical Potential Theory and Its Probabilistic Counterpart, Springer, 1984.

[EG] L. C. Evans and R. F. Gariepy, Wiener's criterion for the heat equation, Arch. Rational Mech. and Analysis 78 (1982), 293-314. 
[FGL] E. B. Fabes, N. Garofalo and E. Lanconelli, Wiener's criterion for divergence form parabolic operators with $C^{1}$-Dini continuous coefficients, Duke Math. J. 59 (1989), 191-232.

[FR1] E.B. Fabes and N.M. Riviere, Symbolic Calculus of Kernels with Mixed Homogeneity, Singular Integrals, A.P. Calderón, Ed., Proc. Symp. Pure Math., Vol. 10, Amer. Math. Soc., Providence, 1967, pp. 106-127.

[FR2] E.B. Fabes and N.M. Riviere, Singular Integrals with mixed homogeneity, Studia Math. 27 (1966), 19-38.

[GZ] Gariepy, Ronald; Ziemer, William P. Thermal capacity and boundary regularity. $J$. Differential Equations 45 (1982), no. 3, 374-388.

[GMT] J. Garnett, M. Mourgoglou and X. Tolsa, Uniform rectifiability from Carleson measure estimates and $\varepsilon$-approximability of bounded harmonic functions, Duke Math. J. 167 (2018), No. 8, 1473-1524.

[GL] N. Garofalo and E. Lanconelli, Wiener's criterion for parabolic equations with variable coefficients and its consequences, Trans. Amer. Math. Soc. 308 (1988), 811-836.

[HL] S. Hofmann and J.L. Lewis, The Dirichlet problem for parabolic operators with singular drift terms, Mem. Amer. Math. Soc. 151 (2001), no. 719.

[HLN] S. Hofmann, J. L. Lewis and K. Nystrom, Duke Math. J. 122 (2004), no. 2, 281346.

[HLe] S. Hofmann and P. Le, BMO solvability and absolute continuity of harmonic measure, The Journal of Geometric Analysis, (2017), 1-22.

[HLMN] S. Hofmann, P. Le, J. M. Martell and K. Nyström, The weak- $A_{\infty}$ property of harmonic and $p$-harmonic measures implies uniform rectifiability, Analysis and PDE 10 (2017), 513-558.

[HM] S. Hofmann and J.M. Martell, Harmonic measure and quantitative connectivity: geometric characterization of the $L^{p}$-solvability of the Dirichlet problem. Part I, preprint.

[HMM1] S. Hofmann, J.M. Martell, and S. Mayboroda, Uniform Rectifiability, Carleson measure estimates, and approximation of harmonic functions, Duke Math. J. 165 (2016), 2331-2389.

[HMM2] S. Hofmann, J.M. Martell, and S. Mayboroda, Transference of scale-invariant estimates from Lipschitz to Non-tangentially accessible to Uniformly rectifiable domains, in preparation.

[HMT] S. Hofmann, J. M. Martell and T. Toro, $A_{\infty}$ implies NTA for a class of variable coefficient elliptic operators, J. Differential Equations, 263 (2017), no. 10, 6147-6188.

[JK] D. Jerison and C. Kenig, Boundary behavior of harmonic functions in nontangentially accessible domains, Adv. in Math. 46 (1982), no. 1, 80-147.

[Ke] C.E. Kenig, Harmonic analysis techniques for second order elliptic boundary value problems, CBMS Regional Conference Series in Mathematics, 83. Published for the Conference Board of the Mathematical Sciences, Washington, DC; by the American Mathematical Society, Providence, RI, 1994.

[KW] R. Kaufmann and J.M. Wu, Parabolic measure on domains of class Lip 1/2 , Compositio Mathematica 65 (1988), 201-207. 
[LSU] O. A. Ladyzenskaja, V. A. Solonnikov, N. N. Ural'ceva, Linear and Quasilinear Equations of Parabolic Type, American Mathematical Society, Providence, R. I., 1967.

[La] E. Lanconelli, Sul problema di Dirichlet per 1Õequazione del calore, Ann. Mat. Pura e Appl., IV 97 (1973), 83-114.

[LM] J. Lewis and M. Murray, The method of layer potentials for the heat equation in time-varying domains, Mem. Amer. Math. Soc. 114 (1995), no. 545.

[L] G. Lieberman, Second Order Parabolic Differential Equations, World Scientific Publishing Co., Inc., River Edge, NJ (1996)

[MT] M. Mourgoglou and X. Tolsa, Harmonic measure and Riesz transform in uniform and general domains, J. Reine Angew. Math., to appear.

[M] J. Moser, A Harnack Inequality for Parabolic Differential Equations, Communications on Pure and Applied Mathematics, Vol. XVII, (1964) 101-134

[N] J. Nash, Continuity of Solutions of Parabolic and Elliptic Equations, American Journal of Mathematics, Vol. 80, No. 4 (Oct., 1958), pp. 931-954.

[NS] K. Nyström and M. Strömqvist, On the parabolic Lipschitz approximation of parabolic uniform rectifiable sets, Revista Matemática Iberoamericana 33 (2017), 1397-1422.

[QX] Zhongmin Qian and Guangyu Xi, Parabolic equations with singular divergence-free drift vector fields, arXiv:1612.07727

[S] S. Semmes (1990). Analysis vs geometry on a class of rectifiable hypersurfaces in Rn, (without bounds on their smoothness). Indiana Math. J., 39, 985-1004.

[SSSZ] Seregin, Gregory; Silvestre, Luis; Šverák, Vladimír; Zlatoš, Andrej On divergence-free drifts. J. Differential Equations 252 (2012), no. 1, 505-540.

[R] N.M. Riviere, Singular Integrals and Multiplier Operators, Ark. Math. 9 (1971), pp. 243-278.

[Su] N. Suzuki, On the essential boundary and supports of harmonic measures for the heat equation, Proc. Japan Acad. Ser. A 56 (1980), 381-385.

[W1] N. Watson, The two versions of the Dirichlet problem for the heat equation, New Zealand Journal of Mathematics 45 (2015), 89-110.

[W2] N. Watson, Introduction to Heat Potential Theory, Mathematical Surveys and Monographs vol.182, Amer. Math. Soc., Providence RI, 2012.

[Z] Zihui Zhao, BMO solvability and the $A_{\infty}$ condition of the elliptic measure in uniform domains, (2017) J. Geom. Analysis Vol. 28, Issue 2, pp. 866-908. 


\section{VITA}

Alyssa Genschaw was born in Evansville, Indiana, but grew up in Marquette, Michigan. She graduated with a bachelor of science degree from Northern Michigan University in 2013, a master of science degree from Northern Illinois University in 2015, and a Ph.D. in mathematics from the University of Missouri in 2019. Her research has appeared in Elemente der Mathematik and in the Journal of Geometric Analysis. Alyssa begins a Visiting Assistant Professor position at the University of Connecticut in August 2019. 